\title{
Dynamic modeling of a solid oxide fuel cell system in Modelica
}

\author{
Daniel Andersson, Erik Åberg
}

Thesis for the Degree of Master of Science
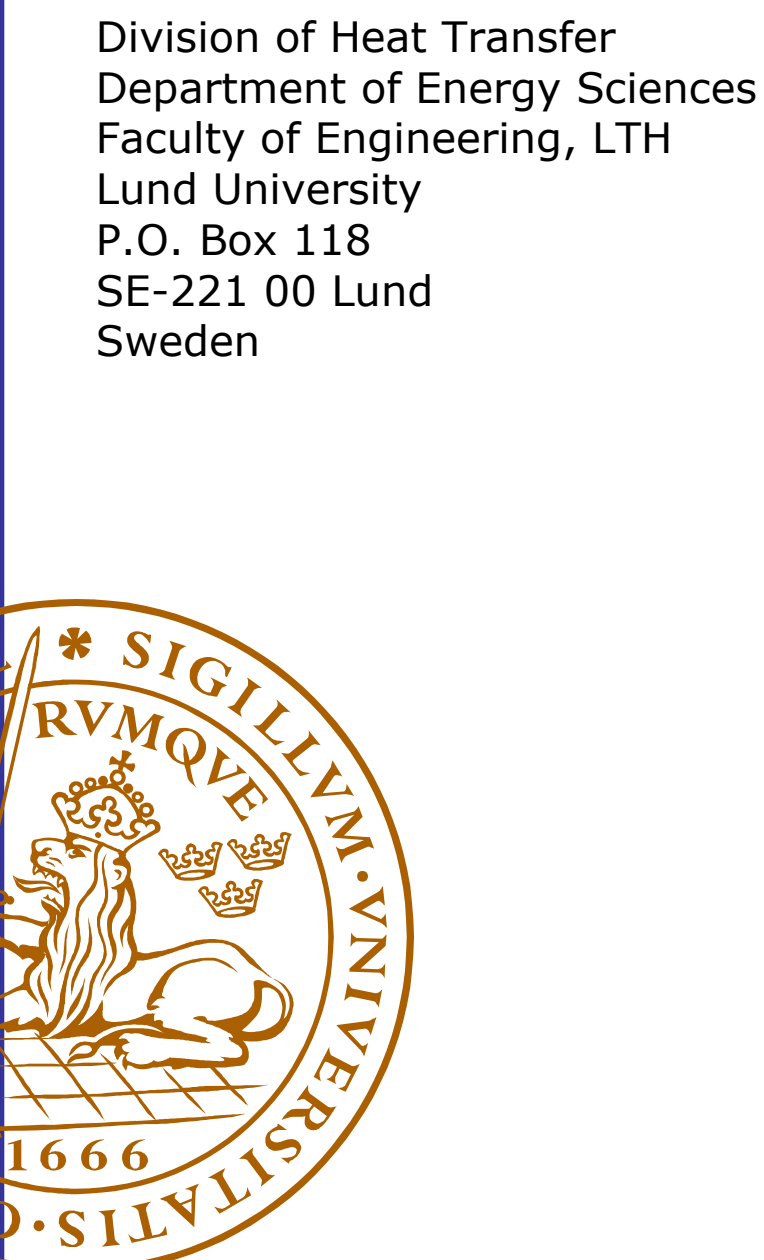



\section{Dynamic modeling of a solid oxide fuel cell}

system in Modelica

Daniel Andersson

Erik Åberg

February 7, 2010 


\begin{abstract}
In this study a dynamic model of a solid oxide fuel cell system has been developed. The work has been conducted in cooperation with Modelon $\mathrm{AB}$ using the Modelica language and the Dymola modeling and simulation tool. Modelica is an equation based, object oriented modeling language, which promotes flexibility and reuse of code. The objective of the study is to investigate the suitability of the Modelica language for dynamic fuel cell modeling. A cell electrolyte model including ohmic, activation and concentration irreversibilities is implemented and validated against simulations and experimental data presented in the open literature. A 1D solid oxide fuel cell model is created by integrating the electrolyte model and a 1D fuel flow model, which includes dynamic internal steam reforming of methane and water-gas shift reactions. Several cells are then placed with parallel flow paths and connected thermally and electrically in series. By introducing manifold pressure drop a stack model is created. The stack model is used in a complete system model including an autothermal reformer, a catalytic afterburner and heat recirculation. Four reactions are modeled in the autothermal reformer. Those are two types of methane steam reforming, the water-gas shift reaction and total combustion of methane.

The simulation results have been compared with those in the literature and it can be concluded that the models are accurate and that Dymola and Modelica are tools well suited for simulations of the transient fuel cell system behaviour.
\end{abstract}




\section{Acknowledgements}

We would like to thank our supervisors, Jonas Eborn at Modelon AB and Dr. Jinliang Yuan at the Department of Energy Sciences. Without their patience answering questions this work would not have been possible. We would also like to thank Hubertus Tummescheit and everyone at Modelon for the nice time there, and all participants in the 2009 Fuel Cell Technology course. Last but not least, thank you family and friends for all support. 


\section{Contents}

1 Introduction 8

1.1 Fuel cells . . . . . . . . . . . . . . . . . 8

1.2 Modelica and Dymola . . . . . . . . . . . . . . . . . . . 9

1.3 Methodology ..................... 9

1.4 Work distribution . . . . . . . . . . . . . . 10

2 Single cell model 11

2.1 Reactions and mass flows .................. 11

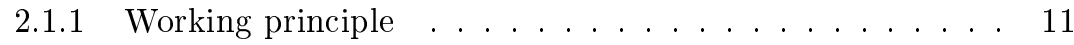

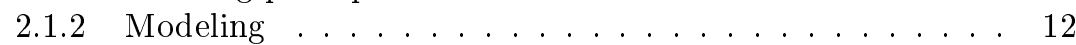

2.1.3 Implementation . . . . . . . . . . . . . . . 13

2.2 Simplified model with ohmic losses only . . . . . . . . . . . 16

2.2.1 Theoretical model . . . . . . . . . . . . . 16

2.2 .2 Implementation . . . . . . . . . . . . . . . . 16

2.3 Complete polarization model . . . . . . . . . . . . . 20

2.3.1 Model overview ................. 20

2.3.2 Ohmic loss model . . . . . . . . . . . . . . 20

2.3.3 Activation loss model ................ 21

2.3.4 Concentration loss model ............. . . 22

2.3.5 Implementation . . . . . . . . . . . . . 23

3 Stack model $\quad 28$

3.1 Substack model . . . . . . . . . . . . . . . . 28

3.1.1 Medium models ......................... 29

3.1 .2 Channel models . . . . . . . . . . . . . . . 29

3.1 .3 Summary . . . . . . . . . . . . . . . . . 30

3.2 Stack model . . . . . . . . . . . . . . . . 32

4 System model $\mathbf{3 5}$

4.1 System layout . . . . . . . . . . . . . . . . 35

4.2 Autothermal reformer . . . . . . . . . . . . . . . . . . 37

4.2.1 Theory .................... 37

4.2.2 Implementation . . . . . . . . . . . . . . . . . . 40

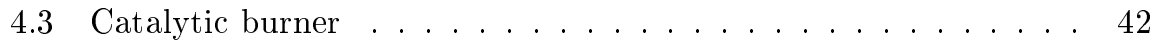

4.4 Other components .................... . . 44

4.4.1 Steam generator ................ . . . . . . . 44

4.4.2 Methane and steam mixing volume . . . . . . . . . . . 44

4.4.3 Heat exchangers ................. . . . . 44

4.5 System model implementation . . . . . . . . . . . . . . . 45

4.5.1 Hotbox model implementation ........... 45

4.5.2 Complete system implementation . . . . . . . . . . 46

5 Results and Discussion $\quad \mathbf{4 7}$

5.1 Setup for investigation of electrical properties . . . . . . . . . 47

5.2 Ohmic loss cell model . . . . . . . . . . . . . . . . . . 48

5.3 Electrochemical cell model . . . . . . . . . . . . . . . 50

5.3.1 Material conductivities ................... 51

5.3.2 Activation loss approximation ........... 51 
5.3.3 Cell pitch length dependency .............. 54

5.3.4 Current collector thickness dependency ......... 54

5.3.5 Effects of varying current density . . . . . . . . . . . 55

5.3.6 Effects of varying temperature . . . . . . . . . . . 56

5.3.7 Effects of varying fuel composition . . . . . . . . . 57

5.4 Substack model validation . . . . . . . . . . . . . . . . 59

5.4.1 Setup for substack model validation . . . . . . . . . 59

5.4.2 Effects of discretization ............. . . 59

5.4.3 Ohmic loss model with increasing current . . . . . . . 61

5.4.4 Electrochemical model startup dynamics . . . . . . . . 64

5.4.5 Electrochemical model with increasing current ..... 68

5.5 Stack model validation . . . . . . . . . . . . . . . . 76

5.6 Verification of system components . . . . . . . . . . . 79

5.6.1 Autothermal reformer ............... . . . 79

5.6.2 Steam generator and fuel mixing ........... . . . 85

5.6.3 Catalytic burner ................. . . 88

5.7 System model validation . . . . . . . . . . . . . . . . . 91

5.7.1 Hotbox validation .................. 91

5.7 .2 System validation ................. 95

6 Conclusions $\quad 99$

6.1 Dymola and Modelica . . . . . . . . . . . . . . . . . . 99

6.2 Cell modeling . . . . . . . . . . . . . . . . . . . . . . 99

6.3 System modeling . . . . . . . . . . . . . . . 100

6.4 Suggestions for future work . . . . . . . . . . . . . . 100 


\section{Nomenclature}

A

$A S R$

$B$

C

$C_{t h}$

$D_{\text {eff }}$

E

F

G

H

I

$i$

$i_{0}$

$J$

$L$

m

$N$

$n_{e}$

$p$

$Q$

R

$r$

$t$

T

U

V

W

$X$

$y$

Greek letters

$\alpha$

$\gamma$

$\eta$

$\lambda$

$\rho$

$\sigma$
Area

Area Specific Resistance

Parameter in Eq.12

Cross-plane resistance area

Thermal mass

Effective diffusion coefficient

Ohmic symmetry factor

Faraday constant

Gibbs free energy

Specific Enthalpy

Electrical current

Current density

Exchange current density

Non-dimensional strip width

Characteristic length

Mass

Number of discretizations

Number of exchanged electrons

Partial pressure

Pressure

Heat

Universal gas constant

Reaction rate

Thickness

Temperature

Internal energy

Voltage

Power

Cell pitch length

Molar fraction $\left[m^{2}\right]$

$\left[\Omega m^{2}\right]$

$\left[\Omega m^{2}\right]$

$[\mathrm{J} / \mathrm{K}]$

$\left[m^{2} s^{-1}\right]$

$\left[C \mathrm{~mol}^{-1}\right]$

$\left[\mathrm{J} \mathrm{mol}^{-1}\right]$

$\left[J \mathrm{~kg}^{-1}\right]$

$\left[A m^{-2}\right]$

$\left[A \mathrm{~m}^{-2}\right]$

$[\mathrm{m}]$

$[k g]$

$[P a]$

$[W]$

$\left[\mathrm{J} \mathrm{mol}^{-1} \mathrm{~K}^{-1}\right]$

$\left[\mathrm{kg} \mathrm{s}^{-1}\right]$

$[\mathrm{m}]$

$[K]$

$[J]$

$[V]$

$[W]$

$\left[\mathrm{mol} \mathrm{mol}^{-1}\right.$ ]

Charge transfer coefficient

Pre-exponential factor (Eq. 21, 22)

Voltage loss

$[V]$

Air / fuel ratio in the catalytic burner

Resistivity

Conductivity
$\left[\Omega^{-1} m^{-1}\right]$ 


$\begin{array}{ll}\text { Subscripts } & \\ \text { a } & \text { Anode } \\ \text { act } & \text { Activation } \\ \text { c } & \text { Cathode } \\ \text { cca } & \text { Current collector of the anode } \\ \text { ccc } & \text { Current collector of the cathode } \\ \text { cell } & \text { Single cell or substack } \\ \text { conc } & \text { Concentration } \\ \text { el } & \text { Electrolyte } \\ \text { flow } & \text { Flow } \\ \text { i } & \text { Component in gas mixture } \\ \text { ref } & \text { Reference, normal temperature or pressure } \\ & \\ \text { Superscrips } & \\ \text { O } & \text { Bulk flow } \\ * & \text { At the reaction site }\end{array}$
Abbreviations
$A T R \quad$ Autothermal reforming
CHP Combined heat and power
$C P O \quad$ Catalytic partial oxidation
$P E M \quad$ Proton exchange membrane
$P O X \quad$ Partial oxidation
ppb Parts per billion
$S O F C$ Solid oxide fuel cell

\section{Chemical}

$\begin{array}{ll}\mathrm{CH}_{4} & \text { Methane } \\ \mathrm{CO} & \text { Carbon monoxide } \\ \mathrm{CO}_{2} & \text { Carbon dioxide } \\ \mathrm{H}_{2} & \text { Hydrogen gas } \\ \mathrm{H}_{2} \mathrm{O} & \text { Water } \\ \mathrm{N}_{2} & \text { Nitrogen gas } \\ \mathrm{O}_{2} & \text { Oxygen gas }\end{array}$




\section{Introduction}

\section{$1.1 \quad$ Fuel cells}

A fuel cell in general is an electrochemical device that produces energy by oxidizing fuel from a replenishable source. Many combinations of fuel and oxidants are possible, but most common is the hydrogen fuel cell. In this case the fuel consists of hydrogen and the oxidant is oxygen. The primary reaction taking place is oxidation of hydrogen

$$
2 \mathrm{H}_{2}+\mathrm{O}_{2} \rightarrow 2 \mathrm{H}_{2} \mathrm{O}
$$

and thus the only byproduct produced aside from heat is water. Hence the fuel cell is a pollution-free power source, provided that the fuel can be produced in a clean way. Thanks to the increasing awareness of the need for environmentally friendly power sources fuel cell technology is developing at a rapid pace.

Many different types of fuel cells exist and they are distinguished by their operating temperature and electrolyte materials. Table 1 lists the most common types of fuel cells and their operating temperature range.

\begin{tabular}{lcr} 
Fuel cell type & Mobile ion & Operating temperature \\
\hline Alkaline (AFC) & $\mathrm{OH}^{-}$ & $50-200^{\circ} \mathrm{C}$ \\
Proton exchange membrane (PEMFC) & $\mathrm{H}^{+}$ & $30-100^{\circ} \mathrm{C}$ \\
Direct methanol (DMFC) & $\mathrm{H}^{+}$ & $20-90^{\circ} \mathrm{C}$ \\
Phosphoric acid (PAFC) & $\mathrm{H}^{+}$ & $220^{\circ} \mathrm{C}$ \\
Molten carbonate (MCFC) & $\mathrm{CO}_{3}^{2-}$ & $650^{\circ} \mathrm{C}$ \\
Solid oxide (SOFC) & $\mathrm{O}^{2-}$ & $500-1000^{\circ} \mathrm{C}$
\end{tabular}

Table 1: Summary of different types of fuel cells

The alkaline fuel cell is used in space vehicles, such as Apollo and the Shuttle Orbiter craft. It uses highly porous electrodes and platinum catalyst. The main problem with the AFC is that the air and fuel supplies must be free from carbon dioxide.

The proton exchange membrane fuel cell utilizes a solid polymer electrolyte and small amounts of platinum catalyst. The PEMFC operates at a low temperature which makes it suitable for vehicles and mobile applications, and possibly $\mathrm{kW}$ sized combined heat and power systems. Negative aspects of the PEMFC is that the hydrogen must be very pure. The catalyst on the PEM anode is gradually deactivated by carbon monoxide in the fuel, and CO levels as low as $1 \mathrm{ppb}$ has been shown to permanently poison a PEM anode catalyst.

The direct methanol fuel cell is essensially a PEMFC operated with methanol as fuel. These fuel cells are very limited in power but may have a future driving portable electronics with low power requirements running for longer time.

The phosphoric acid fuel cell utilizes porous electrodes, platinum catalysts and a higher operating temperature to increase reaction rates. Large numbers of $200 \mathrm{~kW}$ combined heat and power systems are alredy in terrestrial use in 
many parts of the world. Many PAFC systems have demonstrated continuous operation for one year or more with little maintenance.

The molten carbonate fuel cell utilizes a high temperature mixture of lithium, potassium and sodium carbonates in liquid form as electrolyte. The high operating temperature allows for high reaction rates using nickel as catalyst. MCFCs are suitable for medium- to large-scale combined heat and power systems with up to megawatt capacity.

The solid oxide fuel cell is also a high temperature fuel cell, but the electrolyte stays solid during operation. The high temperature removes the need for expensive platinum catalyst and allows gases such as natural gas to be used as fuel directly. SOFCs are suitable for all sizes of combined heat and power applications, from 2 kilowatts to several megawatts. They can also be used in auxiliary power units, or in hybrid systems together with a gas turbine [1-3]

\subsection{Modelica and Dymola}

Modelica is a non-proprietary modeling language with the goal of modeling the dynamical behaviour of technical systems in a convenient way. The language is object-oriented to enable reuse of written code. The models are described by differential, algebraic and discrete equations. There are many tools for development and simulation of Modelica models, both free and commercial, and Dymola is one of them [4].

Dymola is developed by Dynasim AB in Sweden and is a complete tool for modeling and simulation of integrated and complex systems. It is used within automotive, aerospace, robotics, process and other applications [5]. In Dymola, models can be shown and modified both in graphic and text layers. When adding a component or making connections in the graphic layer, corresponding equation or declarations are automatically generated in the text layer. Models are described by equations, typically physical balance equations. Before simulating a model it is compiled and the original equations are reordered and manipulated in order to generate code that is efficient in simulation [6].

\subsection{Methodology}

At the beginning of this project the authors had no previous knowledge about fuel cells and only some experience of modeling in Dymola. Because of this, large amounts of time has gone into getting to understand the working principles of fuel cells and how to model them. To learn about fuel cells we participated in the course Fuel Cell Technology given by Dr. Jinliang Yuan at the Department of Energy Sciences, Faculty of Engineering, Lund University. We have also studied the course book [1] thoroughly and it is cited at several places in this report. To be able to start working with Dymola right away we participated in a three day Dymola course given by Modelon $\mathrm{AB}$ at the beginning of this project.

The amount of fuel cell models with the necessary parameters and simulation data presented in open literature is quite limited and therefore the first part of 
the project was to do a literature study. During this time we studied a lot of published articles on fuel cell modeling, of which only a fraction is actually used as references to the modeling work presented here.

For project management the web-based Trac software has been used, together with the Subversion version control system $[7,8]$.

This report was written using the $\mathrm{H}_{\mathrm{E}} \mathrm{X}$ typesetting system and data plots were created using Matlab. Illustrative figures were created using the GNU image manipulation program GIMP.

\subsection{Work distribution}

Since both of us had no previous knowledge about fuel cells, and only little experience of modeling in Dymola, the first parts of this project had to be done in parallel by both of us. We both studied a lot of literature and papers, developed the single cell models together, and had an ongoing discussion about the working principles of the fuel cell and how to model it.

Later during the study, more and more tasks could be assigned to one of us, but still with an active discussion, of course. The tasks that were performed mostly by one of us are the following:

Performed by Daniel:

- Documentation of the single cell and substack models.

- Development and documentation of the steamgenerator and catalytic burner models.

- Development and documentation of the hotbox model.

- Documentation of the system layout.

- Validation of the single cell and substack models.

- Validation of the hotbox model.

Performed by Erik:

- Development and documentation of the complete stack model.

- Development and documentation of the ATR unit.

- Development and documentation of the system model.

- Validation of the complete stack model.

- Validation of the ATR model.

- Validation of the system model. 


\section{Single cell model}

In this chapter we cover the working principles of the solid oxide fuel cell and modeling of a single cell component.

\subsection{Reactions and mass flows}

\subsubsection{Working principle}

The SOFC is a specific type of fuel cell in which the electrolyte consists of an ion-conducting solid oxide. The working principle of the SOFC is that oxygen, usually from the air, supplied to the cathode side is ionized according to

$$
\mathrm{O}_{2}+4 e^{-} \rightarrow 2 \mathrm{O}^{2-}
$$

The electrons on the left must be supplied to the cathode from an external electrical circuit. The oxygen ions produced are conducted through the electrolyte to the anode where they react with hydrogen according to

$$
2 \mathrm{H}_{2}+2 \mathrm{O}^{2-} \rightarrow 2 \mathrm{H}_{2} \mathrm{O}+4 e^{-}
$$

This is the energy producing reaction in the solid oxide fuel cell. The electrons from the oxygen ions are supplied to the external electrical circuit and the water is produced as steam.

For the SOFC to work the electrolyte material must conduct oxygen ions but isolate electrical current by flow of free electrons. The first such material to be found was zirconia $\left(\mathrm{ZrO}_{2}\right)$, described by Nernst in 1899. The most common electrolyte material in SOFCs of today is zirconia stabilized by addition of yttria $\left(\mathrm{Y}_{2} \mathrm{O}_{2}\right)$, which conduct oxygen ions at temperatures above $800^{\circ} \mathrm{C}$. Therefore SOFCs always have a relatively high operating temperature, often in the range of $800^{\circ} \mathrm{C}$ to $1100^{\circ} \mathrm{C}$ [1]. To allow lower operating temperatures much work is needed to find new materials for the SOFC components, see for example $[9,10]$.

The high operating temperature gives the SOFC disadvantages compared to other types of fuel cells because it must be pre-heated by an external system at start-up, but it also has some positive features. At this high temperature carbon monoxide can be combusted according to

$$
2 \mathrm{CO}+2 \mathrm{O}^{2-} \rightarrow 2 \mathrm{CO}_{2}+4 e^{-}
$$

However the combustion of $\mathrm{CO}$ is not implemented in the model.

It is also possible to internally reform methane to carbon monoxide and hydrogen using the steam produced in equation 3 according to

$$
\mathrm{CH}_{4}+\mathrm{H}_{2} \mathrm{O} \rightarrow \mathrm{CO}+3 \mathrm{H}_{2}
$$

The possibility for these reactions enables the SOFC to run well on methane rich fuels, which is an important advantage compared to other types of fuel cells. In high temperature fuel cells the water-gas shift reaction can also occur. It is given by

$$
\mathrm{CO}+\mathrm{H}_{2} \mathrm{O} \rightarrow \mathrm{H}_{2}+\mathrm{CO}_{2}
$$


The water-gas shift reaction rarely runs to completion, but stops at equilibrium with $0.25-0.5 \%$ carbon monoxide left in the gas [1].

The voltage obtained over a single cell is quite small. A typical voltage over a cell under normal operating conditions is about $0.7 \mathrm{~V}$. To produce a higher voltage many cells are connected in series and forms what is known as a stack. Fuel and air must be supplied to each anode and cathode, and the two flows must be kept separated everywhere in the stack. To deal with those issues several stack designs have been proposed and used. A common design is to connect the electrodes with so called bipolar plates. These connect the electrodes over a large proportion of their surface, giving good electrical properties, and have channels for an evenly distributed supply of air and fuel [1].

\subsubsection{Modeling}

The cell is modeled as an object which reads information of pressure and molar fractions from the bulk flow, generates energy and affects the composition in the anode and cathode channels. The energy is produced in the form of electricity and heat. For the electrical connection the cell has two electrical pins. The heat is distributed to a number of components. It can be kept in the cell itself, increasing its temperature, transfered via convection or conduction to the fuel and air flows or conducted out from the cell as a concequence of an external cooling device.

In this model the cell object is the only place where coupling between the anode and cathode flows can occur. This means that the hydrogen combustion (3) must be modeled here, as it affects the properties of both the anode and cathode bulk flows. In this case the cell object reads the partial pressures of hydrogen and steam in the anode flow and of oxygen in the cathode flow. These pressures are used to determine the hydrogen combustion rate, and then the reactants and products are removed and added to the bulk flows accordingly. The cell object is modeled with zero volume, i.e. no air or fuel is stored in it. When mass is removed from the air at the cathode side it is at the same time added in form of products at the anode side.

The cell models described in this chapter can also be used as simple stack models by setting a parameter $n_{\text {cell }}$ to the number of cells in the stack. This factor is multiplied to the voltages and flows, modeling the stack as a number of identically operating cells. This is of course a crude way of modeling a real SOFC stack, but serves a purpose when simulating entire systems. When simulating a complete system, modeling of individual cells is too detailed. Later in the complete stack modeling, it is also used to model a part of a complete stack, i.e. a substack model.

Figure 1 shows a schematic view of the cell. The anode and cathode boundaries are shown with a current density due to flow of ions through the electrolyte. Externally the current is driven through a load and due to the voltage generated electrical power is supplied to it. Also indicated in the figure are the bulk flows on the anode and cathode side. 


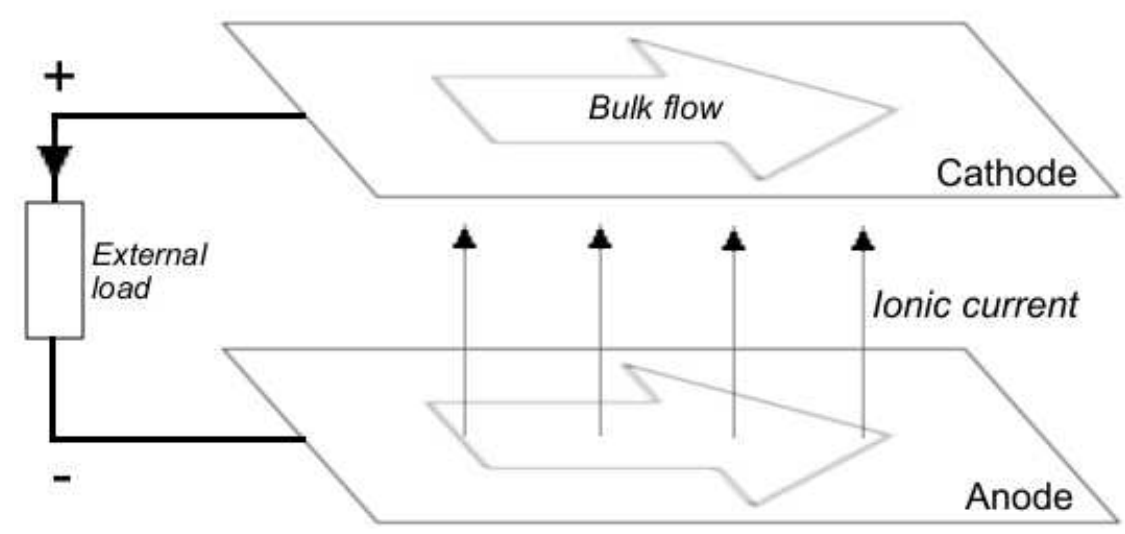

Figure 1: Schematic view of a cell

\subsubsection{Implementation}

To implement the cell model a template model containing common parts in all cell models is created. This includes connectors for mass flow, heat transfer and electrical current, and some balance equations. The fuel and air compositions will have large variations across the cell and to account for this the cell is discretized in the fuel flow direction. The number of discretizations $N$ is a model parameter which can be set before simulation, thereby allowing the user to compromise between model accuracy and simulation time. A schematic view of the discretized cell is shown in Figure 2. In Figure 3 a graphical view of the components is shown. The small blue squares are electrical pins, the round blue connectors are the ports for mass flow and the red square shaped ports are for conductive heat flow.

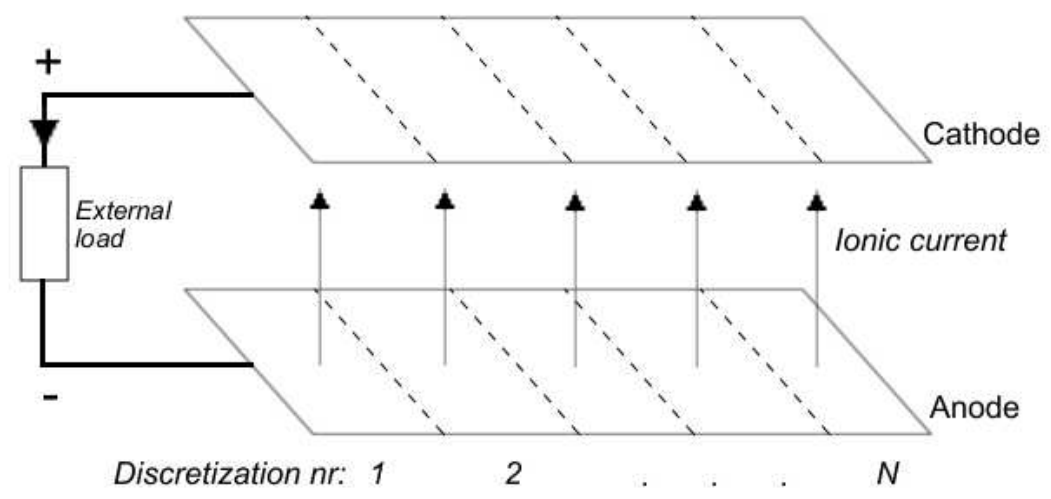

Figure 2: Schematic view of the discretized cell

This model is simply called CellPartial. Table 2 contains the parameters declared in the partial cell model, and Table 3 contains the dynamic states. 


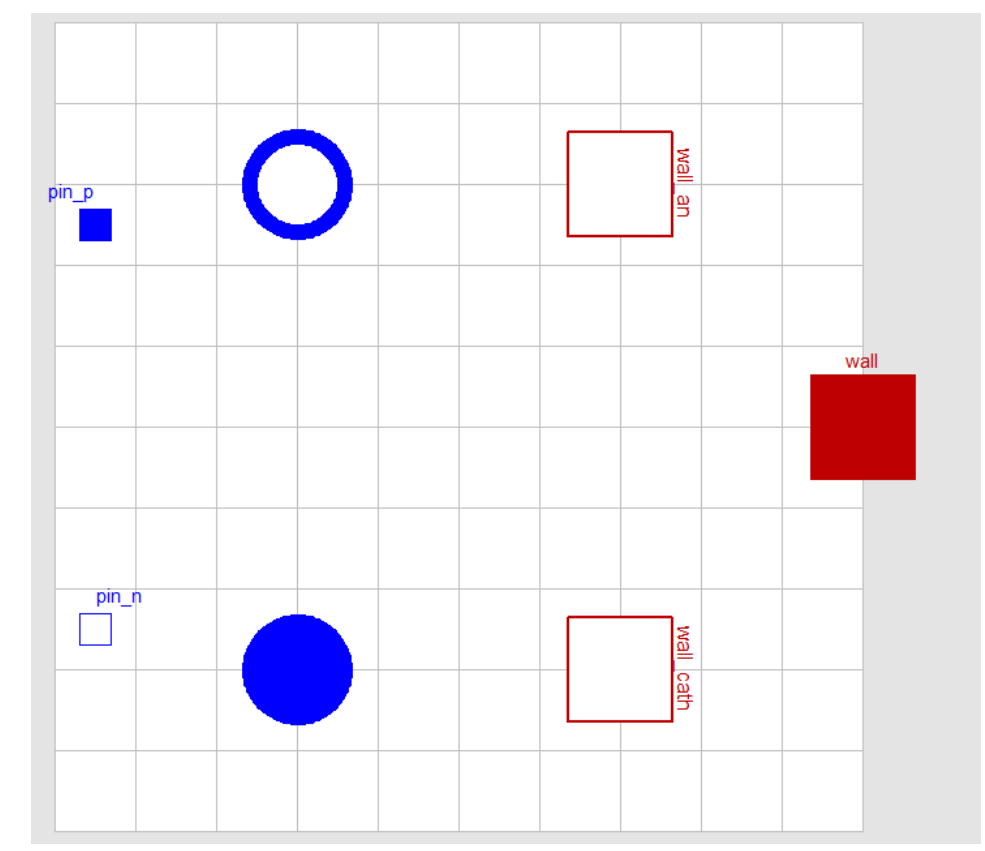

Figure 3: Graphical view of components in the partial cell

An $[N]$ in the table means that the variable is a vector with one element per discretization.

\begin{tabular}{|c|c|c|}
\hline Type & Name & Description \\
\hline integer & $\mathrm{N}$ & Number of discretizations \\
\hline constant Integer & $\mathrm{nX}$ _an & Number of mass fractions in anode \\
\hline constant Integer & $\mathrm{nX}_{-}^{-}$cath & Number of mass fractions in cathode \\
\hline Pressure & pstart & Pressure start value \\
\hline Temperature & Tstart & Temperature start value \\
\hline Area & A_cell & Active cell area \\
\hline Integer & n_cell & Number of cells in stack \\
\hline HeatCapacity & $\overline{\mathrm{MC}} \mathrm{p} \_$cell & Cell heat capacity \\
\hline CoefficientOfHeatTransfer & $\mathrm{kc}$ & Heat transfer coeff. bulk to cell \\
\hline Index & ref & Reformate component indices \\
\hline Index & air & Cathode air component indices \\
\hline
\end{tabular}

Table 2: Parameter and constant declarations in partial cell model

CellPartial also contains a number of equations, such as:

pin_p.v - pin_n.v = V_cell;

This equation sets the cell voltage variable to the difference in electrical potential between the positive and negative pin.

anode $\cdot \mathrm{p}=$ port_an $\cdot \mathrm{p}$; 


\begin{tabular}{|c|c|c|}
\hline Type & Name & Description \\
\hline package & Medium_an & Anode medium model \\
\hline package & Medium_cath & Cathode medium model \\
\hline Temperature[N] & $\mathrm{T}_{\text {_cell }}$ & Cell temperature \\
\hline Voltage & $\mathrm{V}_{-}^{-}$cell & Stack voltage \\
\hline Power & $\mathrm{P}_{-}$total & Total electric power \\
\hline Power[N] & Q_cell & Heat produced in each cell part \\
\hline MoleFraction[N, $n x_{-}$an] & $y_{-}$an & Anode gas molar fractions \\
\hline MoleFraction[N, nx_cath] & $\mathrm{y}_{-}$cath & Cathode gas molar fractions \\
\hline ReactionProperties $[\bar{N}]$ & anode & Porperties of anode gas \\
\hline ReactionProperties[N] & cathode & Properties of cathode gas \\
\hline
\end{tabular}

Table 3: Dynamic state declarations in partial cell model

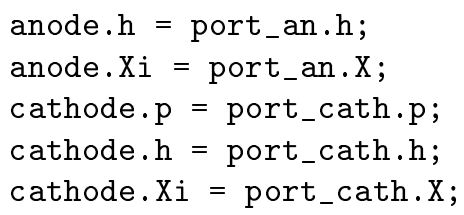

Setting the properties of the local anode and cathode objects equal to values read from the ports.

wall. T = T_cell;

Setting the temperature of the port for external cooling to that of the cell.

wall_an.Q_flow $=\mathrm{kc} * 2 * \mathrm{~A}_{-} \mathrm{cell} /(\mathrm{N}) *\left(\right.$ wall_an.T $\left.-\mathrm{T}_{-} \mathrm{cell}\right)$;

wall_cath.Q_flow $=\mathrm{kc} * 2 * \mathrm{~A}_{-} \mathrm{cell} /(\mathrm{N}) *\left(\right.$ wall_cath. $\left.\mathrm{T}-\mathrm{T}_{-} \mathrm{cell}\right)$;

Setting the heat flow to the bulk proportional to temperature difference.

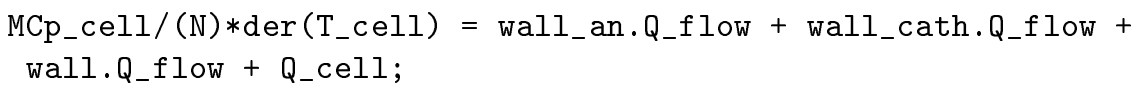

Setting the derivative of temperature times the thermal mass of the discretization to the sum of produced and added heat.

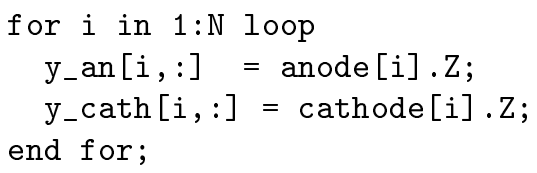

Setting anode and cathode gas molar fraction variables to those of the anode and cathode objects.

P_total $=V_{-}$cell $*$ pin_n.i;

Calculating the electrical power from the cell. 


\subsection{Simplified model with ohmic losses only}

\subsubsection{Theoretical model}

In this case a cell model based on the Nernst potential for the open circuit voltage and an ohmic loss term is developed. This is a highly simplified model, but it serves well as a basic model to which additional effects can be added. It can also be useful in control applications for an entire fuel cell system where only a simple current to voltage behaviour is required for the cell model and short computation time is desirable.

The voltage over the fuel cell without any load is given by the Nernst equation as

$$
V_{\text {Nernst }}=-\frac{\Delta G}{2 F}-\frac{R T}{2 F} \ln \left(\frac{p_{\mathrm{H}_{2} \mathrm{O}} p_{\text {ref }}^{0.5}}{p_{\mathrm{H}_{2}} p_{\mathrm{O}_{2}}^{0.5}}\right)
$$

where $\Delta G$ is Gibbs free energy from the reaction.

When a load is connected and current flows through the cell the voltage drops due to several different effects, one of which is ohmic loss related to resistivity of the fuel cell materials. In this model only an ohmic loss term is implemented, and represents all the voltage losses occuring in the SOFC. Thus the voltage over the cells external connectors is given by

$$
V=V_{\text {Nernst }}-\eta_{\text {Ohmic }}
$$

where $\eta_{\text {Ohmic }}$ is the voltage drop over the internal resistance. The voltage drop is calculated from the cell current density and the area specific resistance according to

$$
\eta_{\text {Ohmic }}=A S R \cdot i
$$

where $A S R$ is the area specific resistance and $i$ the current density due to flow of ions through the elecrolyte. The value of the $A S R$ is modelled as temperature dependent only, according to

$$
A S R(T)=A S R_{0} \exp \left(\frac{E_{a}}{R}\left(\frac{1}{T}-\frac{1}{T_{0}}\right)\right)
$$

where the constant $A S R_{0}$ is the area specific resistance at temperature $T_{0}$ [11].

\subsubsection{Implementation}

The model above is implemented as a discretized cell. This means that for each discrete part there is a voltage source and a resistance defined independently from those of the surrounding parts. All discretizations are then connected to the external pins of the cell, so that the same voltage is obtained over each pair of voltage and resistance. A schematic view is shown in Figure 4 and a graphical view of the components in the model is shown in Figure 5.

This cell model extends the partial cell model defined in chapter 2.1 and will thereby have all declarations end equations defined in it. A new set of variables is added, as given in Tables 4 and 5 .

Equations included in the model are: 


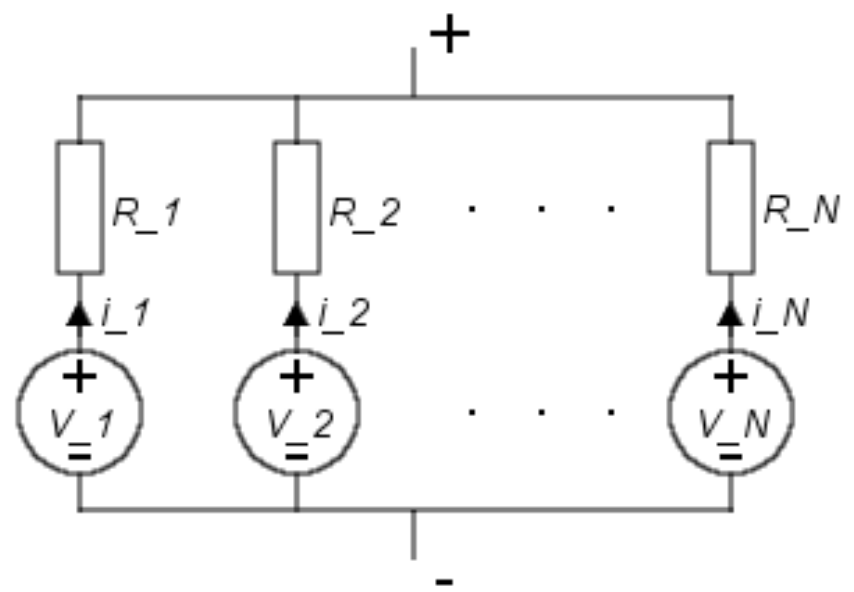

Figure 4: Schematic view of the discretized cell model

\begin{tabular}{|c|c|c|}
\hline Type & Name & Description \\
\hline Power & Q_total & Total heat produced \\
\hline Power[N] & $\mathrm{P}_{-}$cell & Power generated in cell part \\
\hline CurrentDensity[N] & j__Ionic & Current density in cell part \\
\hline CurrentDensity & j_External & Current density out of stack \\
\hline MassFlowRate[N] & $\mathrm{m}$ _flow $\mathrm{O} 2$ & Oxygen flow into cell part \\
\hline MassFlowRate[N] & $\mathrm{m}$ _flowH2 & Hydrogen flow into cell part \\
\hline MassFlowRate[N] & $\mathrm{m}_{-}^{-}$flow $\mathrm{H} 2 \mathrm{O}$ & Steam flow into cell part \\
\hline MassFlowRate[N, nX_an] & $\mathrm{m} \overline{\mathrm{X}} \mathrm{i}$ _flow_an & Ionic mass flow, anode side \\
\hline Voltage [N] & $V_{-}$nernst & Open circuit voltages \\
\hline Resistance[N] & r_ēInt & Internal resistances \\
\hline MolarInternalEnergy[N] & g_reaction & Gibbs free energy from reaction \\
\hline Pressure [N] & $\mathrm{p} \overline{\mathrm{H}} 2$ & Partial pressure of hydrogen \\
\hline Pressure[N] & $\mathrm{pH} 2 \mathrm{O}$ & Partial pressure of steam \\
\hline Pressure[N] & $\mathrm{pO} 2$ & Partial pressure of oxygen \\
\hline
\end{tabular}

Table 4: Declarations made in both models

pH2 = y_an $[:$, ref.H2 $] . *$ anode.p;

pH2O = y_an $[:$, ref.H2O.$*$ anode.p;

p02 = y_cath $[:$,air.02].$*$ cathode.p;

Setting the partial pressures as the molar fractions times the absolute pressure.

g_reaction $=$-anode.g_formation [ref.H2] + anode.g_formation[ref.H20] $0.5 *$ cathode.g_formation [air.02];

Calculating Gibbs free energy of the hydrogen combustion reaction.

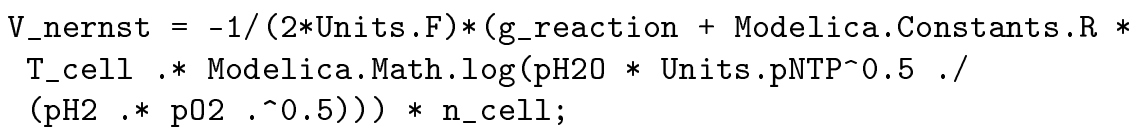




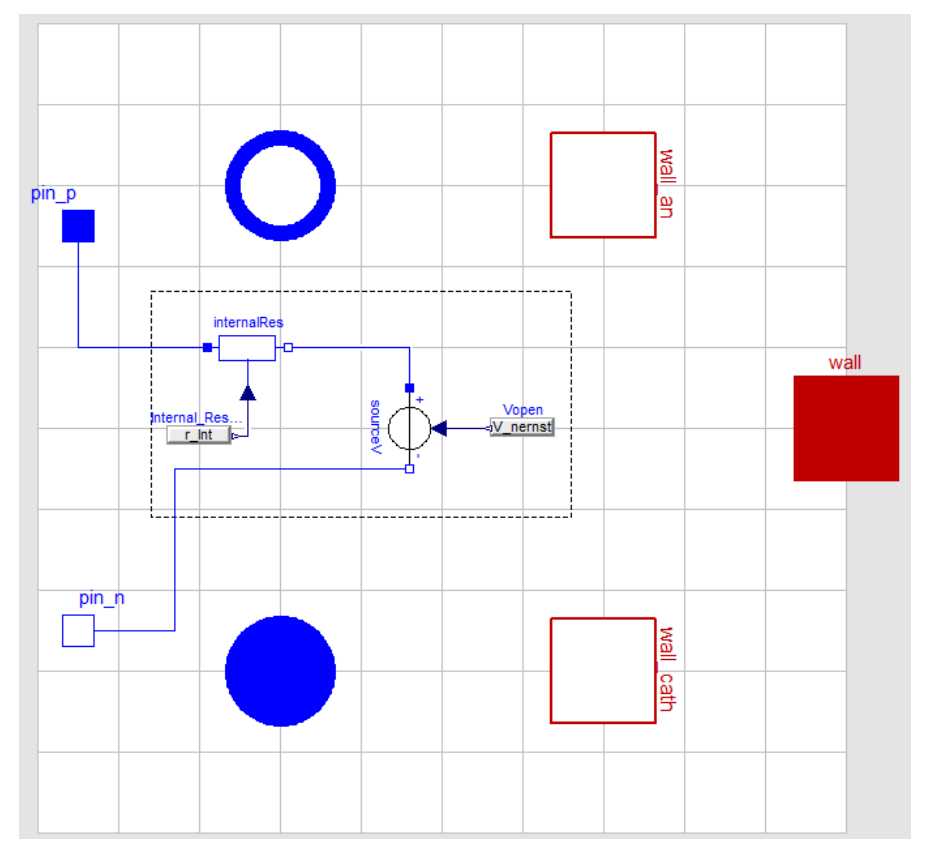

Figure 5: Graphical view of components in the ohmic loss model

\begin{tabular}{llr} 
Type & Name & Description \\
\hline AreaSpecificResistance & ASR0 & Reference in ASR formula \\
Energy & Ea & Activation energy in ASR \\
Temperature & T0 & Temperature reference in ASR \\
Voltage[N] & V_nernst & Open circuit voltages \\
Resistance[N] & r_Int & Internal resistances \\
AreaSpecificResistance[N] & ASR & Area specific resistance
\end{tabular}

Table 5: Declarations made in the ohmic loss model

Calculating the open circuit voltage in all discretizations according to equation 7 .

ASR $=\operatorname{ASRO} * \exp \left((\right.$ Ea/Modelica.Constants.R $) *\left(1 . / T_{-}\right.$cell .- 1/T0) $)$;

Calculating the area specific resistance for each discretization according to equation 10.

r_Int $=\mathrm{n}_{-} \operatorname{cell} * \mathrm{~N} * \mathrm{ASR} / \mathrm{A}_{-} \operatorname{cell}$;

Setting the internal resistance equivalent to the calculated area specific resistance.

pin_n.i $=$ j_External $*$ A_cell;

j_Ionic $=$-internalRes.i $* \mathrm{~N} / \mathrm{A}_{-}$cell;

$P_{-}$cell $=-V_{-}$cell $*$ internalRes.i;

Calculating the current densities and the power generated from each discretization. 


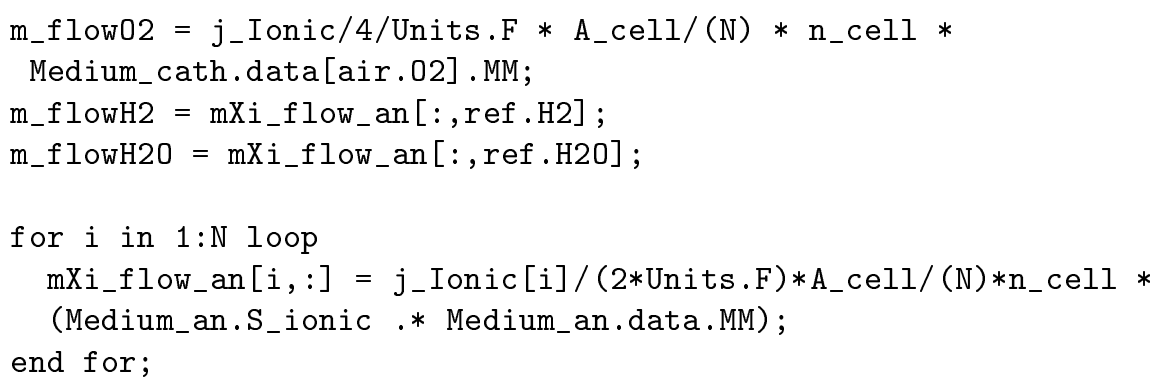

Calculating the mass flow rates for every discretization from their respective ionic current density, and assigns it to the mass flow variables.

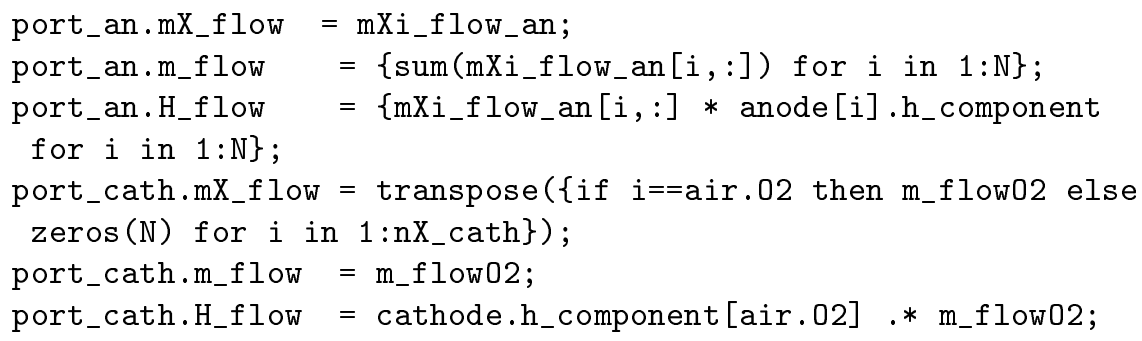

Sets the calculating mass and enthalpy flows to the mass flow ports.

Q_cell = port_an.H_flow + port_cath.H_flow - P_cell;

Calculating the heat produced in each cell discretization from energy balance.

Q_total = sum(internalRes.LossPower) - sum(wall_an.Q_flow) sum(wall_cath.Q_flow) - sum(wall.Q_flow);

Calculating the total heat produced in the cell.

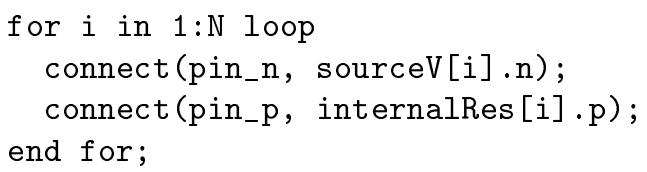

Connects the cell external pins to the pins of each discrete part. 


\subsection{Complete polarization model}

\subsubsection{Model overview}

In this section a complete polarization model is described. The ohmic loss model is extended to include the activation and concentration losses. The activation loss is caused by the slowness of the reactions taking place on the electrodes. A proportion of the generated voltage is requiered for driving the chemical reactions and to transfer the electrons to or from the electrodes, and thereby lost internally in the cell [1].

The concentration loss derives from the change in concentration of reactants and products at the reaction sites, or three phase boundaries, compared to concentrations in the bulk flow. At the reaction sites hydrogen and oxygen is consumed at a rate related to the electrical current through the cell, and water is produced. As it takes some time for new reactants to diffuse through the electrode to the reaction site there will be a decrease in concentration of reactants and an increase of products at this site [1].

To sum up, the total voltage over the cell is given by [12]

$$
V=V_{\text {Nernst }}-\eta_{\text {Ohmic }}-\eta_{\text {act }}-\eta_{\text {conc }}
$$

\subsubsection{Ohmic loss model}

The calculation of the ohmic loss is based on the formulas for the ohmic resistance of a PEN strip with diagonal terminals, which is based on the cell geometry in Figure 6.

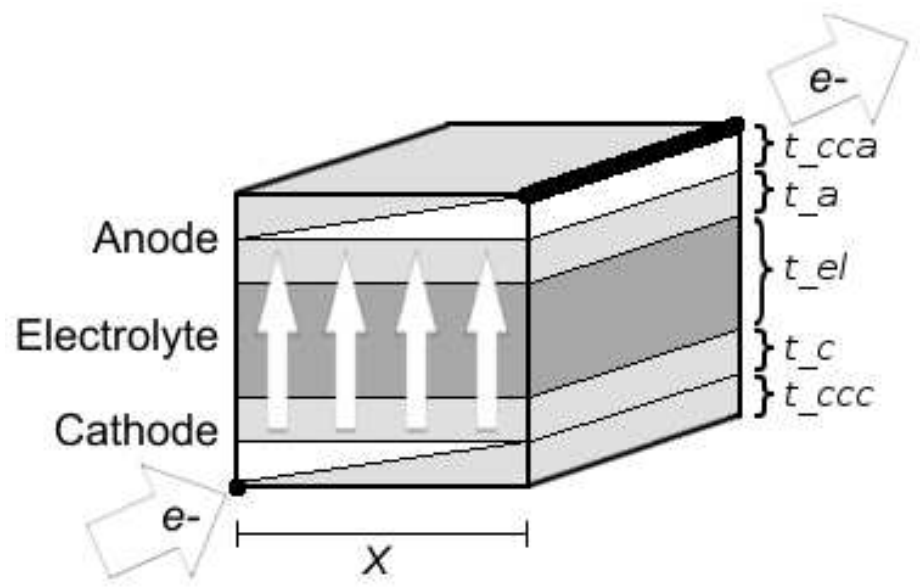

Figure 6: Geometry of the cell for ohmic resistacne calculation

The white fields show the current path through the cell. The electrons enter the cross-section at the cathode terminal at the lower left, indicated by the arrow marked $e^{-}$. The electrons are then distributed via both cross-plane and in-plane flow through a current collector to the active cathode sites, where the oxygen is ionized. From there the current is conducted in cross-plane paths in form of oxygen ions through the electrolyte to the active anode sites. From 
the active area the electrons are conducted through a current collector at the anode side via both in-plane and cross-plane flow to the terminal at the upper right where the electrons exit the cell, as indicated with an arrow marked $e^{-}[13]$.

For this geometry the area specific resistance is given by

$$
A S R=C J\left[\operatorname{coth}(J)+B\left(J-\tanh \left(\frac{1}{2} J\right)\right)\right]
$$

where $C$ is the cross-plane resistance area, given by

$$
C=\rho_{c} t_{c}+\rho_{c c c} t_{c c c}+\rho_{e l} t_{e l}+\rho_{a} t_{a}+\rho_{c c a} t_{c c a}
$$

$B$ is given by

$$
\begin{array}{r}
B=\frac{E}{(1+E)^{2}} \\
E=\left(\frac{t_{c c a}}{\rho_{c c a}}+\frac{t_{a}}{\rho_{a}}\right)^{-1}\left(\frac{t_{c c c}}{\rho_{c c c}}+\frac{t_{c}}{\rho_{c}}\right)
\end{array}
$$

where $E$ is the ohmic symmetry parameter. $J$ is given by

$$
L=\sqrt{\frac{\rho_{e l} t_{e l}}{\left(\frac{t_{c c a}}{\rho_{c c a}}+\frac{t_{a}}{\rho_{a}}\right)^{-1}+\left(\frac{t_{c c c}}{\rho_{c c c}}+\frac{t_{c}}{\rho_{c}}\right)^{-1}}}
$$

where $X$ is the cell pitch length as indicated in Figure 6 [12].

The ohmic conductivity of the materials included are temperature dependent on the form [12]:

$$
\sigma=\rho^{-1}=\beta_{1} \exp \left(-\frac{\beta_{2}}{T}\right)
$$

\subsubsection{Activation loss model}

The cell voltage drop known as the activation loss derives from the fact that the reacting species in chemical reactions must overcome an energy barrier, i.e. the activation energy for the reaction. The activation loss can be seen as an extra potential required to drive the reaction at a rate that would be expected if there were no energy barrier to overcome [14].

The activation loss has a nonlinear current dependency and a parameter known as the exchange current density, denoted $i_{0}$, is of great importance. With no current through the cell there are still reactions taking place on the electrode surface, but the reactions also occur backwards at the same rate. Thus, even if there is no net current, there is sill a flow of electrons from and to the electrode and this flow is the exchange current density. Therefore, when applying a current density lower than the exchange current density, one is only shifting a current that is already present. However, when applying a current density greater than the exchange current density, a new current is introduced in the 
material.

For current densities $i<i_{0}$ the activation loss is low, typically a few hundredths of a volt, and for current densities $i>i_{0}$ the activation loss grows according to the Tafel equation:

$$
\eta_{a c t}=\frac{R T}{2 \alpha F} \ln \left(\frac{i}{i_{0}}\right)
$$

In this equation a new parameter $\alpha$ is also included, which is known as the charge transfer coefficient to measure how large proportion of the applied electrical energy contributes to changing the reaction rate [1].

For current densities $i<i_{0}$ the activation loss is assumed to be proportional to the logarithm of the current density on the form:

$$
\eta_{\text {act }}=k \cdot \ln i
$$

The slope $k$ is choosen so that the activation loss reaches a reasonable value for $i=i_{0}$. This linear function and the Tafel expression are then merged smoothly at their intersection using the Dymola built-in function spliceFunction. It generates a function with a smooth hyperbolic transition between the two expressions, and can be used as an expression for the activation loss for all current densities [15].

The activation loss must be calculated for both the anode and cathode side, which have different exchange current densities, denoted $i_{0, a}$ and $i_{0, c}$. They are calculated from the Arrhenius law and the composition of the reacting gases according to [12]:

$$
\begin{array}{r}
i_{0, c}=\gamma_{c}\left(\frac{p_{\mathrm{O}_{2}}}{p_{\text {ref }}}\right)^{0.25} \exp \left(-\frac{E_{a c t, c}}{R T}\right) \\
i_{0, a}=\gamma_{a}\left(\frac{p_{H_{2}}}{p_{\text {ref }}}\right)\left(\frac{p_{H_{2} O}}{p_{\text {ref }}}\right) \exp \left(-\frac{E_{a c t, a}}{R T}\right)
\end{array}
$$

\subsubsection{Concentration loss model}

The electrochemical reactions take place at the interfaces of the electrolyte and electrodes. This means that the reactants must diffuse through the porous electrodes from the bulk flow to reach the reaction sites, and the products must diffuse back to the bulk flow. Because of this the actual pressure of the reactants and product differs from those in the bulk flow, and the voltage obtained over the cell is lower than that obtained if the reaction site pressure were the same as in the bulk flow. Typically this loss is very small until the current density approaches a limiting current. If a current above this limit is applied the concentration loss will have a severe impact on the cell performance [14].

The concentration loss can be expressed as a function of the effective partial pressures of the reactants and products at the reaction sites as [12]:

$$
\eta_{\text {conc }}=-\frac{R T}{n_{e} F}\left[\ln \left(\frac{p_{H_{2}}^{*} p_{H_{2} O}^{O}}{p_{H_{2}}^{O} p_{H_{2} O}^{*}}\right)+\frac{1}{2} \ln \left(\frac{p_{O_{2}}^{*}}{p_{O_{2}}^{O}}\right)\right]
$$


or, can be modified to a function of the molar fraction $y_{i}$ of the gases:

$$
\eta_{\text {conc }}=-\frac{R T}{n_{e} F}\left[\ln \left(\frac{y_{\mathrm{H}_{2}}^{*} y_{\mathrm{H}_{2} \mathrm{O}}^{O}}{y_{\mathrm{H}_{2}}^{O} y_{\mathrm{H}_{2} \mathrm{O}}^{*}}\right)+\frac{1}{2} \ln \left(\frac{y_{\mathrm{O}_{2}}^{*}}{y_{\mathrm{O}_{2}}^{O}}\right)\right]
$$

There are two terms representing the losses at the anode and cathode sides. The molar fractions at the reaction sites can be calculated from the molar fractions in the bulk, the current density through the electrolyte, the thickness of the anode and cathode, the bulk pressure and the diffusion coefficient as below [12]:

$$
\begin{array}{r}
y_{\mathrm{O}_{2}}^{*}=1+\left(y_{\mathrm{O}_{2}}^{O}-1\right) \exp \left(\frac{i R T t_{c}}{4 n_{e} F D_{\mathrm{O}_{2} P} P}\right) \\
y_{\mathrm{H}_{2}}^{*}=y_{\mathrm{H}_{2}}^{O}-\frac{i R T t_{a}}{2 n_{e} F D_{H_{2}} P} \\
y_{\mathrm{H}_{2} \mathrm{O}}^{*}=y_{\mathrm{H}_{2} \mathrm{O}}^{O}+\frac{i R T t_{a}}{2 n_{e} F D_{\mathrm{H}_{2} \mathrm{O}} P}
\end{array}
$$

Gas molecular diffusion is proportional to the concentration gradient of a species and the diffusion coefficient, according to the Ficks law

$$
J=-D \nabla \phi
$$

where $J$ is the diffusion flux and $\phi$ is the concentration. The diffusion coefficient for a component depends on the gas composition, and if Knudsen diffusion is neglected it can be calculated from the diffusion coefficients of binary systems as

$$
D_{A, g m}=\frac{1-X_{A}}{X_{B} / D_{A B}+X_{C} / D_{A C}+\cdots}
$$

where $D_{A, g m}$ is the diffusion coefficient of component $A$ in the gas mixture containing components $B, C, \ldots$ and $X_{A}, X_{B}, X_{C}, \ldots$ are the molar fractions of the components. $D_{A B}, D_{A C}, \ldots$ are the diffusion coefficients in the $A B, A C, \ldots$ binary systems [16].

\subsubsection{Implementation}

Also for this model the schematic and graphical views in Figure 4 and 5 apply. The differences are, as described above, the way how the internal resistance is calculated and two additional voltage loss terms are included. The model extends the partial model described in chapter 2.1 and also declares the extra variables listed in Tables 4, 6 and 7 .

The equations in this model are enlisted and described below:

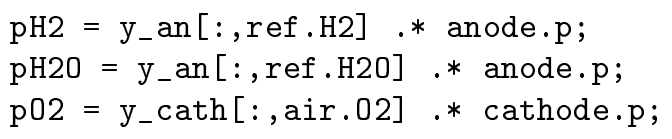

Sets partial pressures as molar fraction times pressure. 


\begin{tabular}{|c|c|c|}
\hline Type & Name & Description \\
\hline Real & $\mathrm{n}_{-} \mathrm{e}$ & Number of exchanged electrons \\
\hline CurrentDensity & gamma_a & Factor in eq. (22) \\
\hline CurrentDensity & mma_c & Factor in eq. (21) \\
\hline MolarInternalEnergy & E_act_a & Activation energy in eq. (22) \\
\hline MolarInternalEnergy & E_act_c & Activation energy in eq. (21) \\
\hline Real & alpha_a & Charge transfer coeff. in eq. (19) \\
\hline Real & alpha_c & Charge transfer coeff. in eq. (19) \\
\hline Conductivity & $\mathrm{ta}-\overline{1} \_\mathrm{a}$ & Constant in eq. (18) \\
\hline Tempe & ta_2_a & Constant in eq. (18) \\
\hline Condr & a_1_el & Constant in eq. (18) \\
\hline Temp & ta_2_el & Constant in eq. (18) \\
\hline Cond & ta_1_c & Constant in eq. (18) \\
\hline Temp & ta_2_c & Constant in eq. (18) \\
\hline Resis & eccà & Resistivity of current collect \\
\hline Resi. & rho_ccc & Resistivity of current collecto \\
\hline Pres & p_ref & Reference pressure in eq. (21), (22) \\
\hline Leng & $\mathrm{X}$ & Cell pitch length \\
\hline Length & $t_{-} \mathrm{c}$ & Thickness of cathode \\
\hline Length & t_el & Thickness of electrolyte \\
\hline Leng & $t_{-}^{-} a$ & Thickness of anode \\
\hline Leng & $t_{-}$ & Thickness of current colles \\
\hline Length & t_ccc & Thickness of current collector cathode \\
\hline Voltag & spliceValue & arameter used in activation calculation \\
\hline DiffusionCoefficient & D_eff_c & Diffusion coefficient of oxygen in air \\
\hline DiffusionCoefficient & D_CH4_H2 & Binary diffusion coefficient \\
\hline DiffusionCoefficient & $\mathrm{D}_{-}^{-} \mathrm{CO} \_\overline{\mathrm{H}} 2$ & Binary diffusion coefficient \\
\hline Diffusion & D_H2O_H2 & Binary diffusion coefficient \\
\hline DiffusionCoefficient & $\mathrm{D}_{-}^{-} \mathrm{CO} 2{ }_{-}^{-} \mathrm{H} 2$ & Binary diffusion coefficient \\
\hline DiffusionCoefficient & $\mathrm{D} \_\mathrm{CH} 4{ }_{-}^{-} \mathrm{H} 2 \mathrm{O}$ & Binary diffusion coefficient \\
\hline DiffusionCoefficient & D_CO_H2O & Binary diffusion coefficient \\
\hline DiffusionCoefficient & D_CO2_H2O & Binary diffusion coefficient \\
\hline
\end{tabular}

Table 6: Parameter declarations made in the model with activation and concentration loss

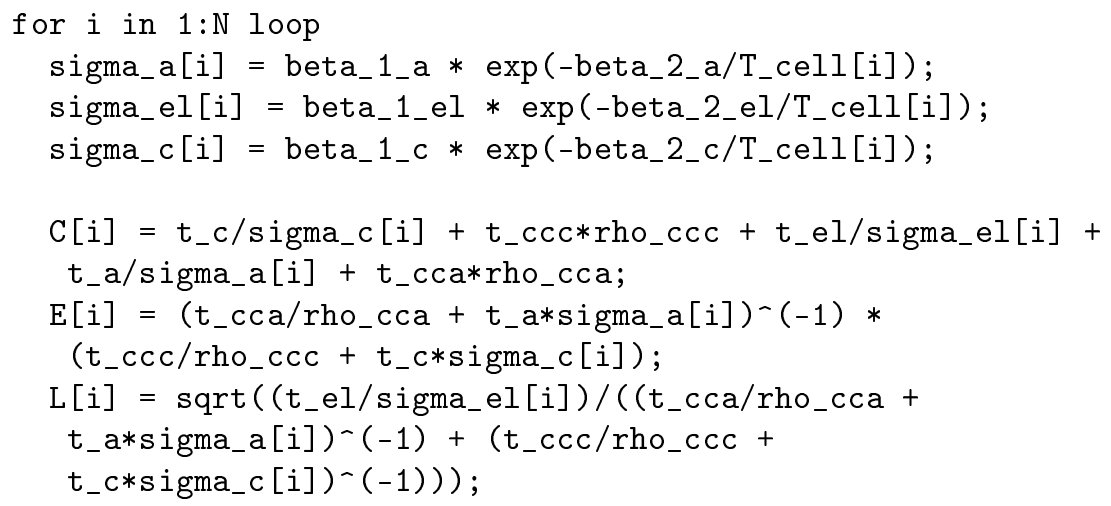




\begin{tabular}{llr} 
Type & Name & Description \\
\hline Conductivity[N] & sigma_a & Anode conductivity \\
Conductivity[N] & sigma_el & Electrolyte conductivity \\
Conductivity[N] & Sigma_c & Cathode conductivity \\
AreaSpecificRes.[N] & C & Cross-plane resistance area \\
Real[N] & E & Ohmic symmetry factor \\
Length[N] & $\mathrm{L}$ & Characteristic length \\
Real[N] & $\mathrm{B}$ & Symmetry parameter \\
Real[N] & $\mathrm{J}$ & Strip width \\
AreaSpecificRes.[N] & $\mathrm{R}$ & Area specific resistance \\
CurrentDensity[N] & i_0c & Exchange current density \\
CurrentDensity[N] & i_0a & Exchange current density \\
Voltage[N] & eta_act & Total activation loss \\
Voltage[N] & eta_act_an & Anode activation loss \\
Voltage[N] & eta_act_cath & Cathode activation loss \\
Voltage[N] & tafel_an & Anode tafel activation loss \\
Voltage[N] & tafel_cath & Cathode tafel activation loss \\
Voltage[N] & linear_an & Anode linear activation loss \\
Voltage[N] & linear_cath & Cathode linear activation loss \\
Voltage[N] & eta_conc & Concentration loss \\
MoleFraction[N] & yH2_reac & Hydrogen mole frac. at reaction site \\
MoleFraction[N] & yH2O_reac & Steam mole frac. at reaction site \\
MoleFraction[N] & yO2_reac & Oxygen mole frac. at reaction site \\
DiffusionCoefficient & D_H2_gm & Hydrogen diffusion coefficient \\
DiffusionCoefficient & D_H2O_gm & Steam diffusion coefficient
\end{tabular}

Table 7: Dynamic state declarations made in the model with activation and concentration loss

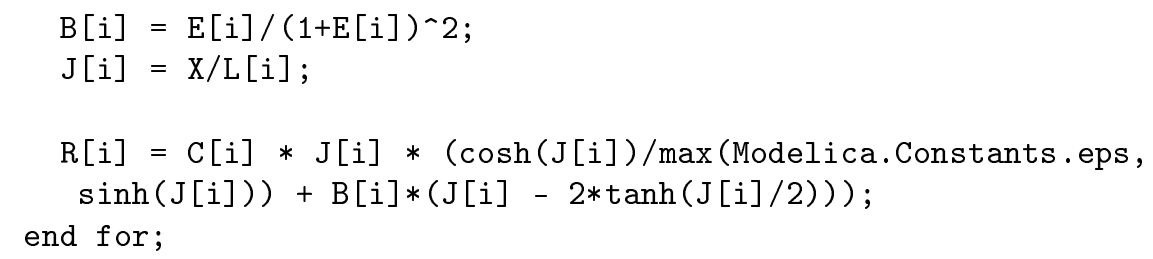

The first expression calculates the temperature dependant conductivities according to equation (18) and the last expression calculates the area specific resistance according to equations (12) to (17).

$\mathrm{r}_{-}$Int $=\mathrm{n}_{-} \operatorname{cell} * \mathrm{~N} * \mathrm{R} / \mathrm{A}_{-} \operatorname{cell}$;

Calculates the internal resistance from the area specific resistance using the area of the discretization and the number of cells in the stack.

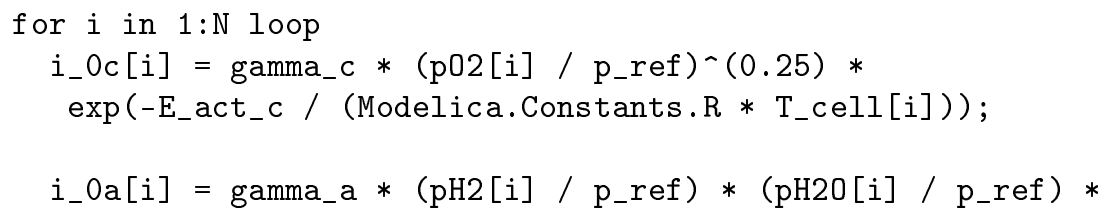




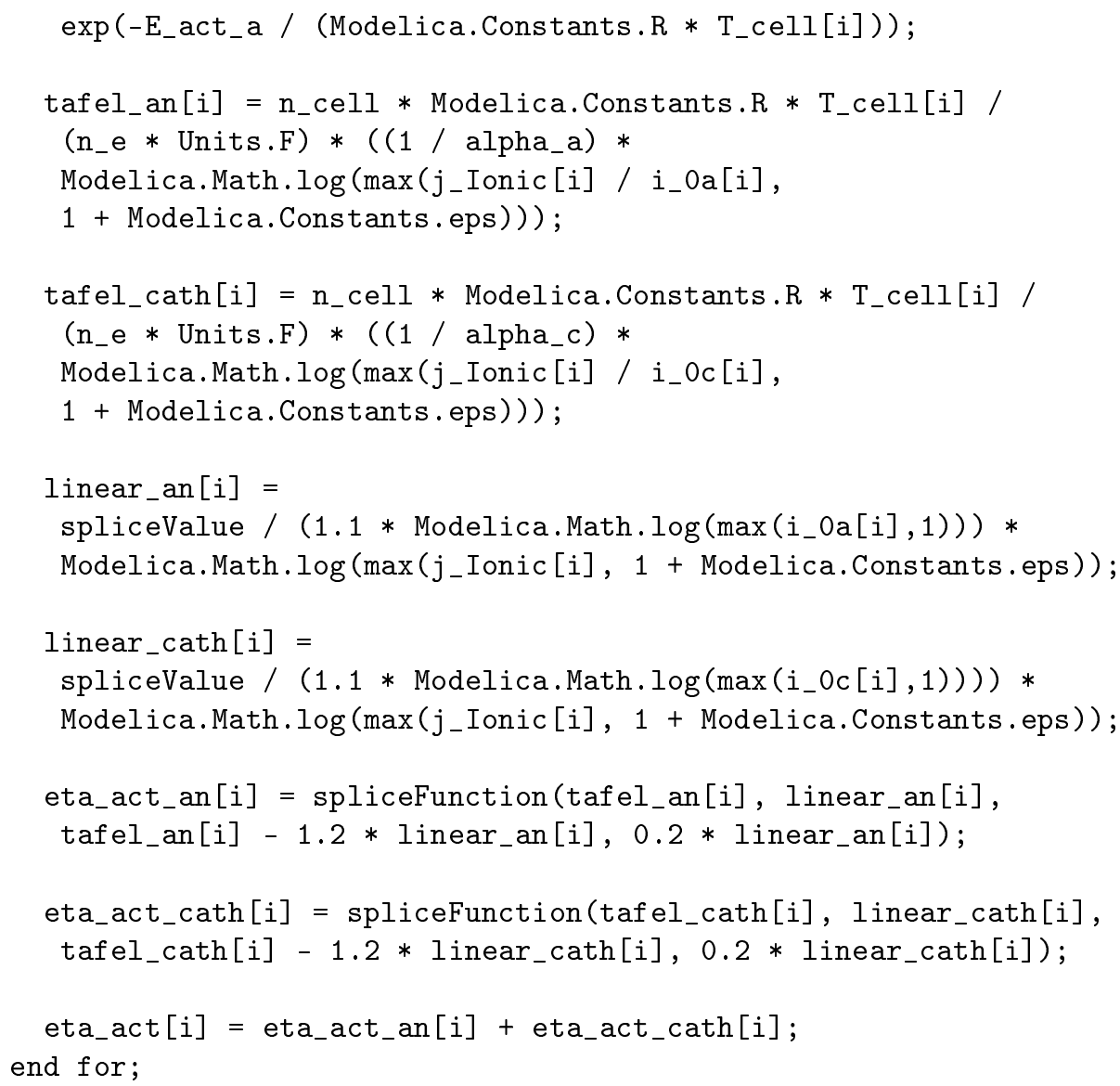

The first expression calculates the exchange current densities according to equations (21) and (22). Then the activation losses both from the tafel equation (19) and the linear approximation (20) are calculated. The results are merged to a function that is valid for all currents using the spliceFunction function. The final expression adds the anode and cathode losses to a total activation loss.

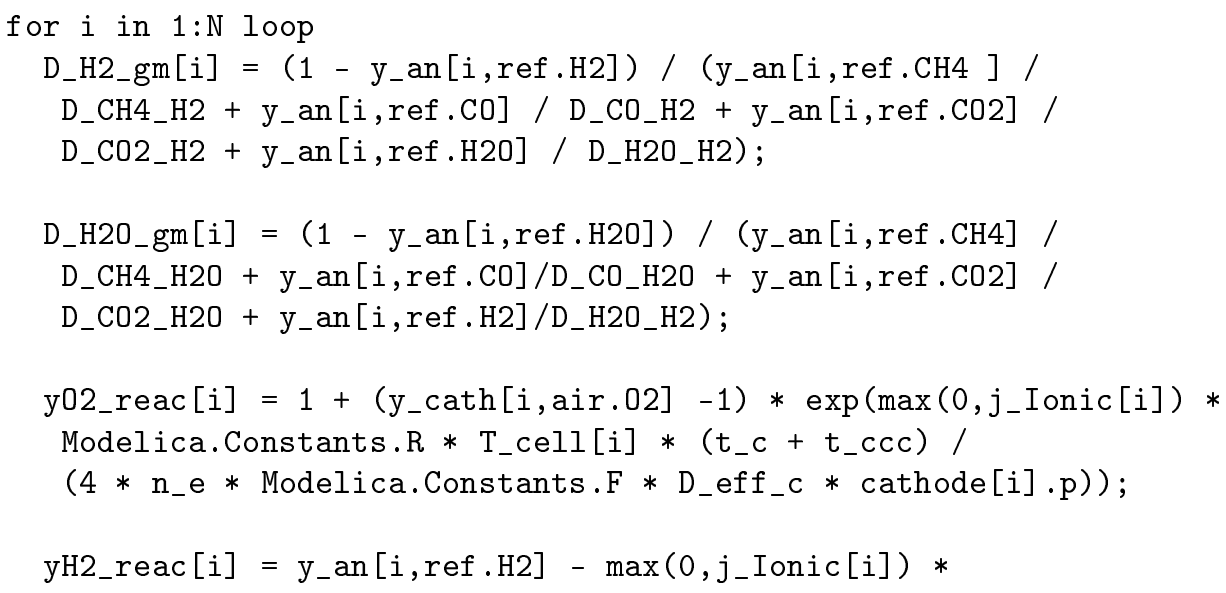




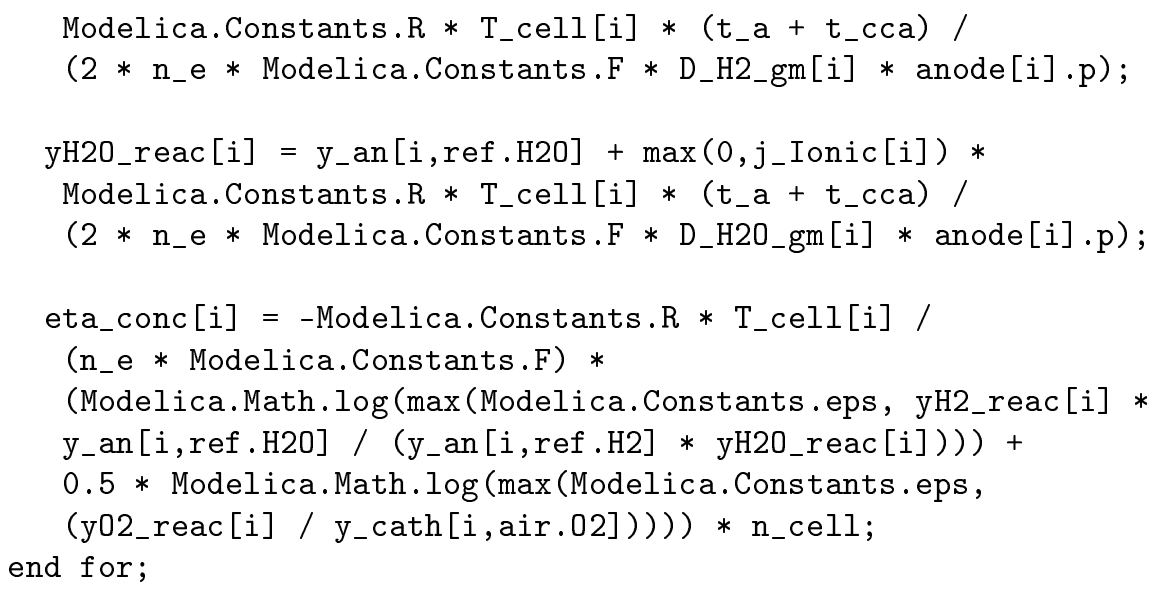

The first expression calculates the diffusion coefficients for hydrogen and steam according to equation 29, then the molar fractions at the reaction site for oxygen, hydrogen and steam are calculated according to equations (25) - (27). Finally the concentration loss is calculated according to equation (24).

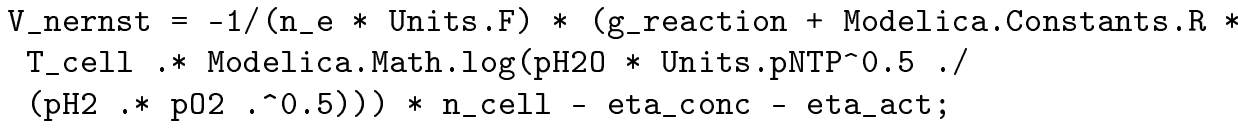

Calculates the Nernst voltage with activation and concentration loss terms included.

Equations for Gibbs free energy, current densities, mass flows, heat production and electrical pin connections are also included. They are not modified from the above mentioned simplified model with only ohmic loss, as in chapter 2.2 . 


\section{Stack model}

This chapter covers modeling of a substack containing one cell model and two flow channel models. The substack model can be used to model an entire stack or to model a part of a stack. Modeling of a complete stack by connecting several substack models in series and including manifold models is also covered.

\subsection{Substack model}

Figure 7 shows the graphical layout of the substack model. In the middle of it there is a replaceable cell model which is constrained so that any model extending the partial model defined in chapter 2 easily can be inserted into it.

Above and below the cell model are models for the flow channels for fuel and air. These objects keep track of and calculate all the bulk medium properties in all discretizations, and in the anode channel also the reforming reaction (5) and the water-gas shift reaction (6) take place.

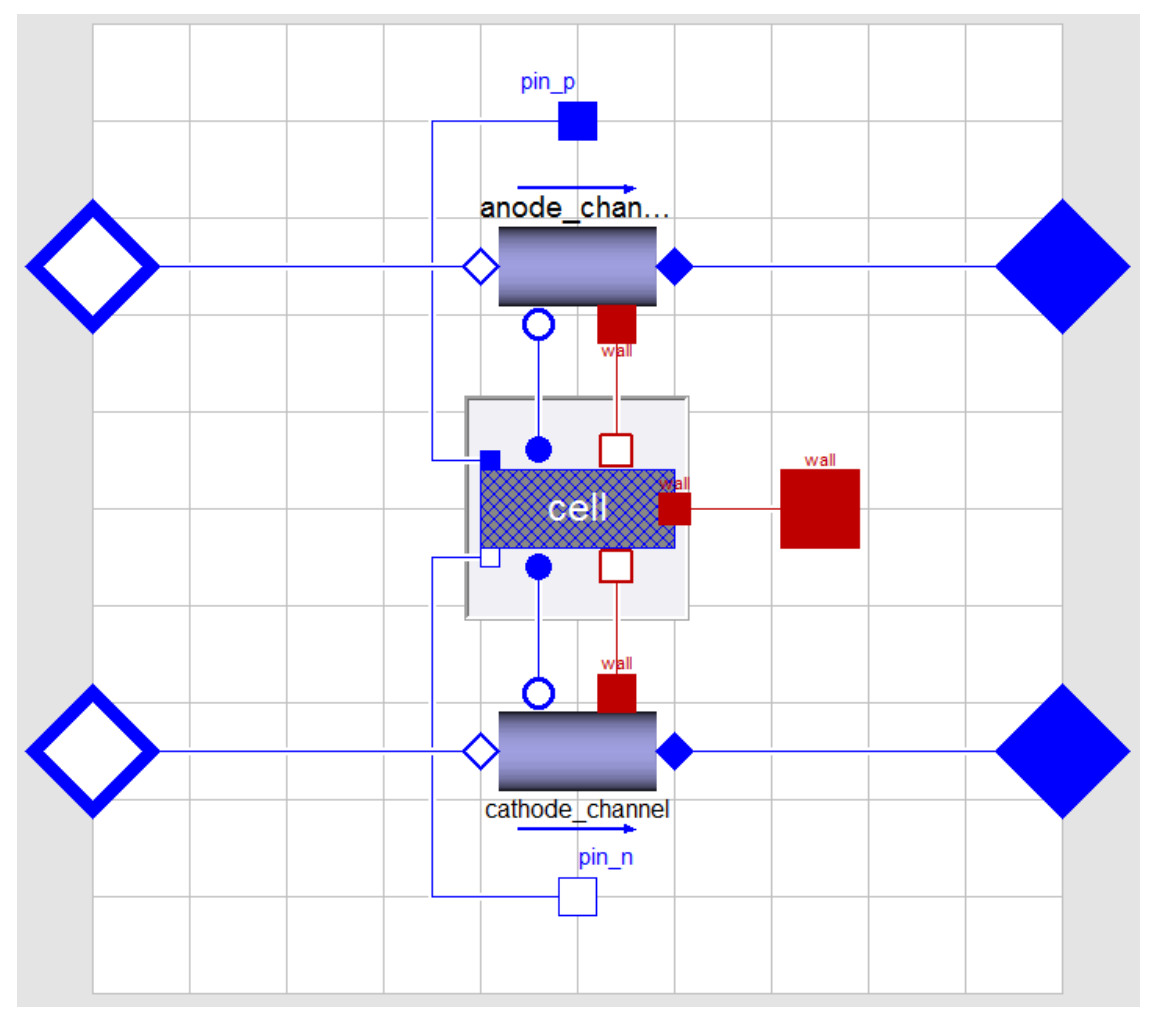

Figure 7: Graphical layout of substack model 


\subsubsection{Medium models}

All components containing a medium, for example the flow channels, calculates the medium properties by including a medium model. The medium model keeps track of all medium parameters and physical data of the components. The meduim model also calculates medium properties from the dynamic states of the volume model.

In a volume where a reaction takes place more details about the medium is required, such as the specific enthalpy, Gibbs free energy and entropy. Those properties are calculated in a medium properties model. The idea is that properties of a medium that are not required in a specific part of the system model should not be calculated at that place. Several medium property models for various applications have been implemented at Modelon. The air model used in this project is a previously developed model, whereas the fuel model is a newly implemented one. The main difference of the fuel medium model compared to previously available ones is the inclusion of more components. This was necessary to be able to mix fuel and air in the ATR unit and in the catalytic burner. The medium models used contain the components shown in Table 8.

\begin{tabular}{lcc} 
Index & Fuel component & Air component \\
\hline 1 & $\mathrm{H}_{2}$ & $\mathrm{Ar}$ \\
2 & $\mathrm{CH}_{4}$ & $\mathrm{CO}_{2}$ \\
3 & $\mathrm{CO}$ & $\mathrm{H}_{2} \mathrm{O}$ \\
4 & $\mathrm{CO}_{2}$ & $\mathrm{~N}_{2}$ \\
5 & $\mathrm{H}_{2} \mathrm{O}$ & $\mathrm{O}_{2}$ \\
6 & $\mathrm{~N}_{2}$ & \\
7 & $\mathrm{O}_{2}$ &
\end{tabular}

Table 8: Components in the fuel and air medium models

\subsubsection{Channel models}

The channel models are based on those previously developed at Modelon. The channel is modeled as a discretized volume, where every discrete volume has a unique medium composition and energy content. The mass flow between the volumes are calculated from the pressure difference. In every volume on the anode side there are two dynamic reactions taking place, the steam reforming reaction and the water-gas shift reaction. The channel is discretized in the same number of discretizations as the cell model, and the connections between the cell model and the channel model are made per discretization. This means that in the cell model every discrete part has a unique fuel and air state to be evaluated and it will result in varying operating conditions across the cell.

The dynamic reactions at the anode are characterized by a reaction object that is included in the channel model. The reaction object contains the stoichiometry matrix defining the reactions and also calculates the equilibrium constants of the reactions. In this case the reactions modeled are the steam reforming reaction (5) and the water-gas shift reaction (6), which are defined by the stoichiometry matrix: 


$$
S_{\text {reaction }}=\left(\begin{array}{c}
S_{\text {reform }} \\
S_{w g s}
\end{array}\right)=\left(\begin{array}{ccccccc}
3 & -1 & 1 & 0 & -1 & 0 & 0 \\
1 & 0 & -1 & 1 & -1 & 0 & 0
\end{array}\right)
$$

Each row in the stoichiometry matrix defines one reaction by giving the number of products with positive sign and the number of reactants with negative sign. The components are ordered according to the index in the medium model, as given in Table 8 .

The first step in determining the rate of the reactions is to determine the equilibrium constant of the reaction. If a reaction is given by:

$$
\nu_{A} A+\nu_{B} B \rightleftharpoons \nu_{C} C+\nu_{D} D
$$

where $\nu_{X}$ is the stoichiometric coefficient of species $X$ in the reaction, the equilibrium constant $K$ of the reaction is given by:

$$
K \equiv e^{-\Delta G / R T}=\frac{p_{C}^{\nu_{C}} p_{D}^{\nu_{D}}}{p_{A}^{\nu_{A}} p_{B}^{\nu_{B}}}
$$

where $\Delta G$ is Gibbs free energy from the reaction and $p_{X}^{\nu_{X}}$ is the partial pressure of component $X$ raised to the power of the stoichiometric coefficient of $X[17]$. In the reaction object the equilibrium constants are calculated from Gibbs free energy from the reactions, which are evaluated from the medium model. The actual reaction rate is then calculated from the current deviation from the equilibrium position.

\subsubsection{Summary}

Finally some useful variables are defined in the substack model, which can be used for plotting and by other models containing the substack model. Those variables are presented in Table 9 .

\begin{tabular}{lr} 
Name & Description \\
\hline T_stack & Stack temperature \\
V_stack & Stack voltage \\
utilization & Fuel hydrogen utilization \\
utilization_CH4 & Fuel methane utilization \\
steam_carb_ratio & Steam to carbon ratio of fuel at inlet
\end{tabular}

Table 9: Variables declared in substack model

The stack voltage and temperature are simply set equal to the voltage and temperature variables of the cell model. The utilization ratio is defined as the ratio of moles of hydrogen consumed to moles of hydrogen at the input. The following expression was initially used to calculate the utilization:

$$
\text { utilization }=\frac{\mathrm{H}_{\text {inflow }} \text { in } \mathrm{H}_{2}, \mathrm{CH}_{4} \text { and } \mathrm{H}_{2} \mathrm{O}-\mathrm{H}_{\text {outflow }} \text { in } \mathrm{H}_{2} \text { and } \mathrm{CH}_{4}}{\mathrm{H}_{\text {inflow }} \text { in } \mathrm{H}_{2}, \mathrm{CH}_{4} \text { and } \mathrm{H}_{2} \mathrm{O}}
$$

However, this expression is not correct because all hydrogen in the inlet steam is not actually available for water-gas shift reforming. A correct utilization expression can instead be given from the net water production according to: 


$$
\text { utilization }=\frac{\mathrm{H}_{\text {outflow }} \text { in } \mathrm{H}_{2} \mathrm{O}-\mathrm{H}_{\text {inflow }} \text { in } \mathrm{H}_{2} \mathrm{O}}{\mathrm{H}_{\text {inflow }} \text { in } \mathrm{H}_{2} \text { and } \mathrm{CH}_{4}}
$$

In this expression the net hydrogen consumption is calculated from the net water production and only the hydrogen in the $\mathrm{H}_{2}$ and $\mathrm{CH}_{4}$ molecules are considered available for consumption. When comparing the two expressions, (33) turned out to give higher values than (34), but the error decreased for larger utilizations. Unfortunately some plots were created using the incorrect expression, and this is stated in the descriptions of the plots.

utilization_CH4 is simply the percentage of methane consumed and steam_carb_ratio is ratio of moles of $\mathrm{H}_{2} \mathrm{O}$ to total moles of carbon atoms in the fuel at the inlet. 


\subsection{Stack model}

A complete stack is modeled by elecrically connecting several substack models in series. The incoming fuel flow is fed to a volume and is then split up to run in parallel through the substacks. A schematic view of the fuel flow is shown in Figure 8 .

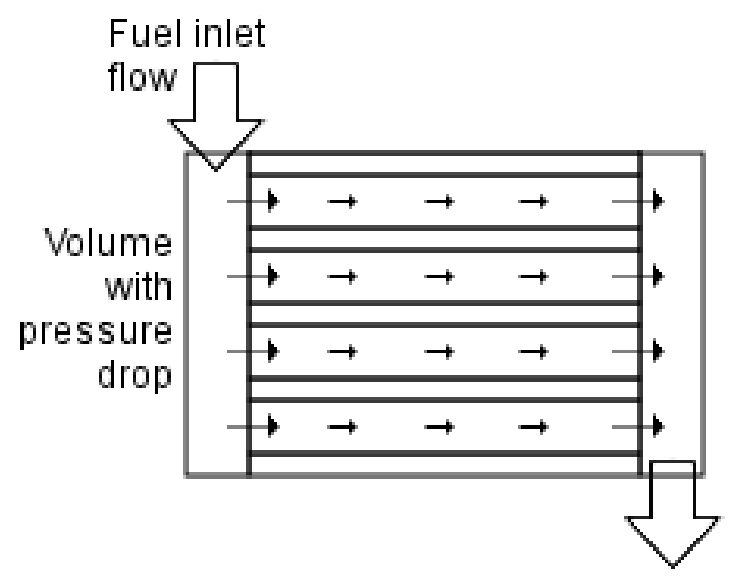

Figure 8: Schematic view of fuel flow in stack with manifold model

The volumes at the stack inlet and outlet correspond to the manifold of the real SOFC stack. Fuel is assumed to be fed at the top of the manifold and because of fuel flow out of the manifold into the cells there will be a small pressure drop along the manifold volume. Compared to the pressure drop inside the cell, however, this pressure drop can be assumed to be small and for this reason we do not model this drop as a discretized volume. Instead the manifold is represented by a single volume model with a single pressure, temperature and fuel composition. The flow into each substack is derived from the difference in pressure between the manifold and the first discretization of the substack channels. When evaluating the pressure in the manifold volume a small proportion is substracted depending on which drain port the pressure is evaluated at, thereby a slightly smaller flow into the lower channels is obtained. The pressure drop is assumed to be linear along the manifold and is defined by a model parameter giving the total pressure drop between the fuel inlet port and the last drain port.

The graphical layout of the model is shown in Figure 9. In the middle there is a vector of substack models, allowing the user to model the stack with an arbitrary number of substack objects.

Not visible in the graphic layer is connections from every substack's heat port to a heat transfer object and then to the next substack object in the vector. This models heat transfer between the different parts of the stack. The first and last substack objects in the vector correspond to top and bottom layers of the stack and are thermally connected to wall objects with a specific heat capacity, modeling the metal casing around the fuel cell. Also not visible in the graphic layer is the electrical connections between the substack objects in the 


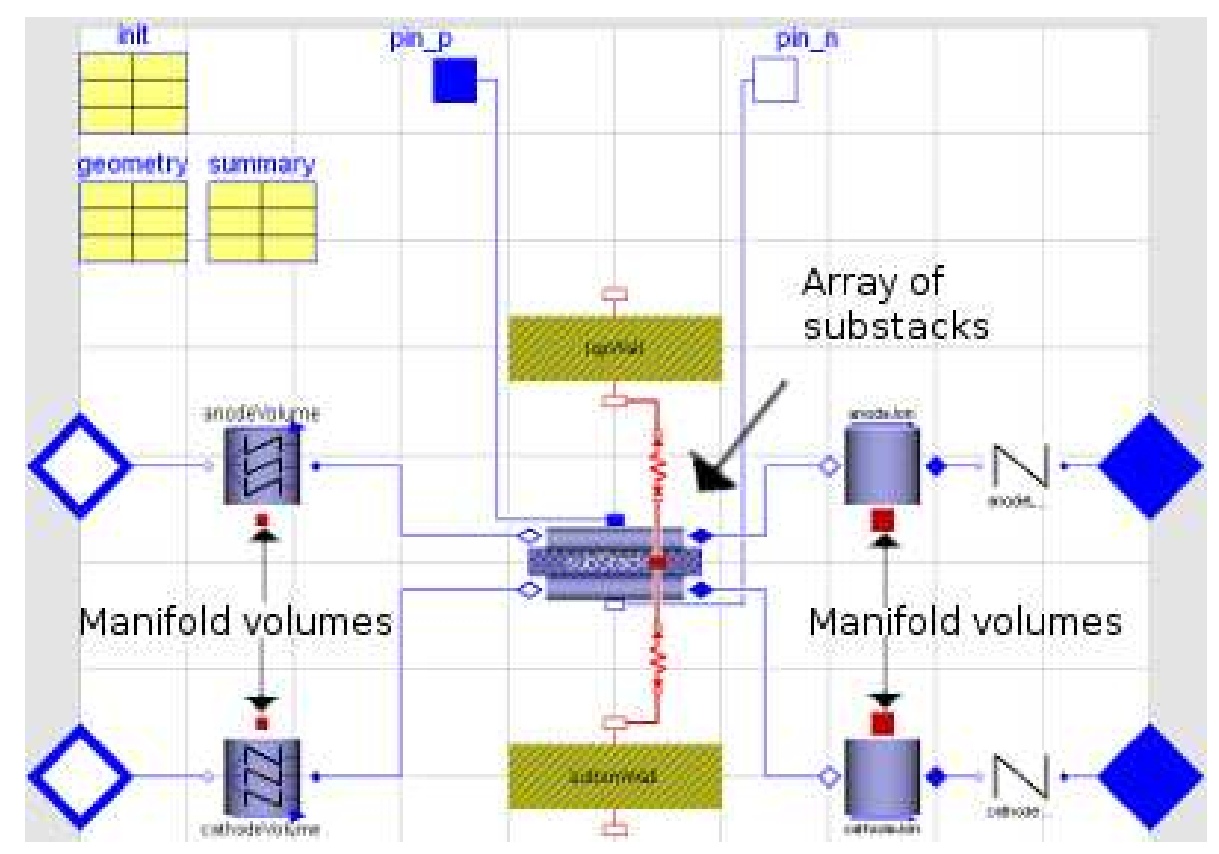

Figure 9: Graphical layout of stack model

vector. The positive pin of the first substack is connected to an external positive pin. Its negative pin is connected to the next substack's positive pin and so on until the last substack whose negative pin is connected to an external negative pin. These connections are made in the text layer of the model as follows:

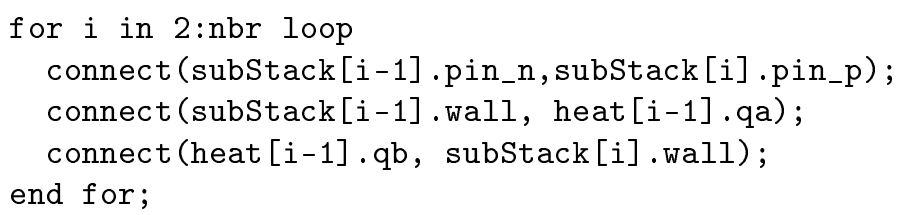

The fuel and air inlets at the left side of the figure are connected to the manifold volumes. The manifold volume models visible in the figure contains both a volume model with a single input and vector of outputs with the same length as the substack vector and a vector of pressure drop models. Each output from the volume model is connected to a seperate pressure drop model. The outputs from these models are then connected to the external connector of the manifold volume model. The result of this setup is that the inlet pressure of each subsubstack is evaluated in a pressure drop model. By comparing the evaluated pressure with the pressure in the volume model a unique inlet flow rate is calculated for every substack.

The outlets of the substack are connected to the same type of volume models used in the inlet manifold, but this time with a vectorized inlet port and a single outlet. Thus the outputs from all the substacks are mixed before exiting the stack. The volume is connected to a pressure drop model before it is connected 
to an external connector, and thus the stack outlet must be connected to an external volume model, where a pressure can be evaluated to determine the outlet flow rate.

Finally there are three data records. Two of them, named init and geometry, are used to input stack initialization and geometry data respectively. This is useful as parameters for objects in the model can be set at the top layer. The last record, called summary, reads important values from model objects and presents them at one place, making it easier to find them when evaluating simulation results. 


\section{System model}

\subsection{System layout}

A SOFC stack can be used in many different types of systems depending on the application. If electrical power generation is the only purpose of the system the efficiency can be increased by using the SOFC together with a gas turbine. In [3] such a system is proposed. In this case the exhaust gases from the anode and cathode channels are fed to a combustor which produces a high temperature and high pressure gas. This gas is fed to a gas turbine which drives two compressors that increases the pressure of the air and fuel before entering the stack. After leaving the gas turbine the combustor exhaust is fed to a power turbine where some of the remaining pressure is converted to electrical energy. After the turbines the remaining hot exhaust gas is fed through two heat exchangers, heating the fuel and air prior to entering the stack. A similar system is presented in [18].

Another application is to use the SOFC for combined heat and power production. A system for such an application is presented in [11]. In this case the anode exhaust gas is burned in an afterburner together with fresh air. The heat produced is used to heat water, which can be circulated in a heat distribution system. The cathode exhaust is used to heat the air prior to entering the stack.

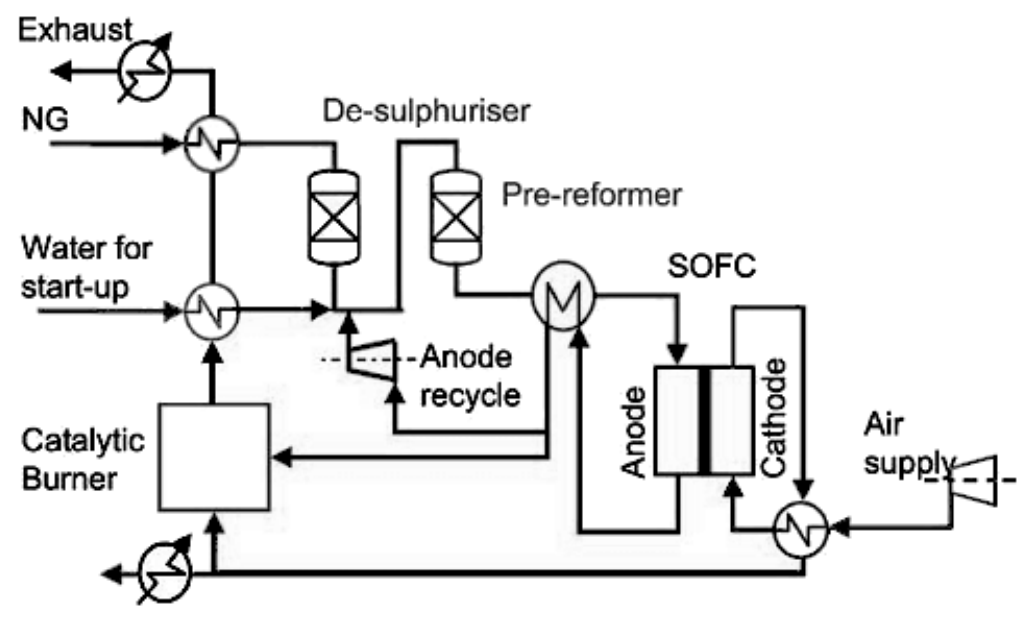

Figure 10: Design of a $250 \mathrm{~kW}$ CHP system [19]

In this project the system design is based on that presented in [19] as shown in Figure 10. The system can be used in CHP applications, but the primary use of the heat from the burner is to evaporate water to steam which is needed in the reformer, and to pre-heat the natural gas from ambient temperature to a temperature suitable for the reformer inlet. The cathode exhaust is used to heat air from ambient temperature before entering the stack, then used in the burner. Any remaining air can be used to heat water for heat distribution. The stack in [19] is of $250 \mathrm{~kW}$ and the net electric efficiency obtained is $55.9 \%$. The total efficiency including the heat available for distribution is $84.7 \%$. 
However the system implemented in this project has some differences from that described in [19]. The natural gas is pre-reformed in an ATR unit, as described in chapter 4.2. This has the advantages of higher energy efficiency than steam reforming and partial oxidation and a higher $\mathrm{H}_{2} / \mathrm{CO}$ ratio which is also easily regulated by the inlet composition [20].

The fuel feedstock is assumed to be a mixture of methane and carbon dioxide, which means that reforming of longer hydrocarbons and desulphurization is not included in the model. The layout of the implemented system is shown in Figure 11.

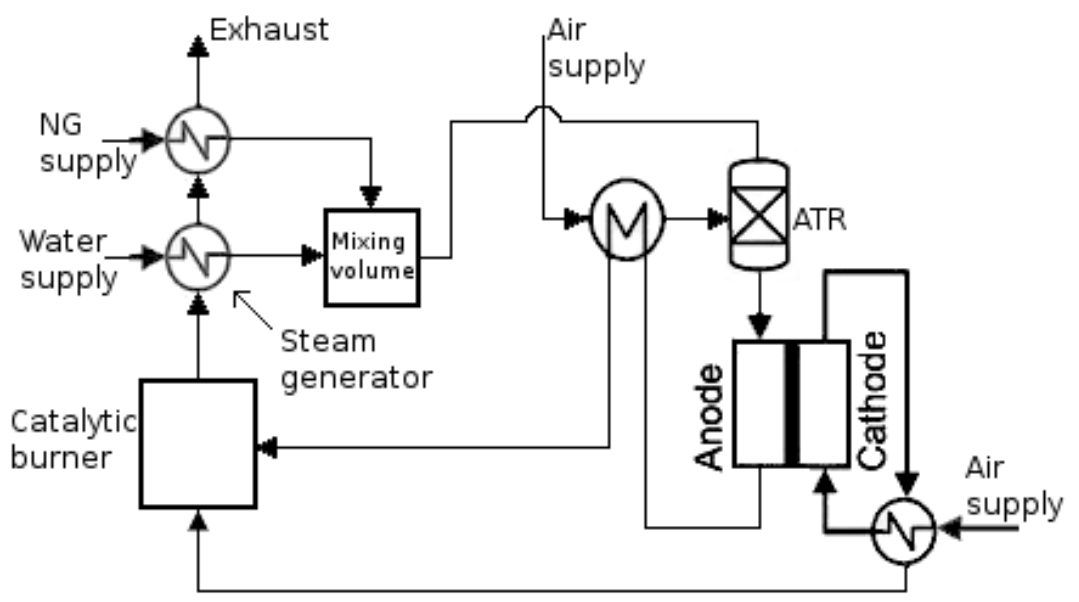

Figure 11: Layout of implemented fuel cell system

As the ATR unit is ideally operated at temperatures above $500^{\circ} \mathrm{C}$ and the net heat production is low, the air must be heated before entering the ATR. This is done by feeding the anode exhaust gas through a heat exchanger, heating the air before the off gas enters the burner. The ATR unit produces a fuel with suitable composition and temperature and can be fed directly to the stack. 


\subsection{Autothermal reformer}

\subsubsection{Theory}

Natural gas contains a high mole fraction of methane and needs to be prereformed before it can be used as fuel in a SOFC stack. Different types of pre-reforming units can be used for this purpose. A steam reforming unit is operated in a temperature range of $850-950^{\circ} \mathrm{C}$ and uses the steam reforming and the water-gas shift reactions to produce hydrogen $[1,20]$ :

$$
\begin{aligned}
\mathrm{CH}_{4}+\mathrm{H}_{2} \mathrm{O} \leftrightarrow \mathrm{CO}+3 \mathrm{H}_{2} & \Delta H=206.2 \mathrm{~kJ} / \mathrm{mol} \\
\mathrm{CH}_{4}+2 \mathrm{H}_{2} \mathrm{O} \leftrightarrow \mathrm{CO}_{2}+4 H_{2} & \Delta H=164.9 \mathrm{~kJ} / \mathrm{mol} \\
\mathrm{CO}+\mathrm{H}_{2} \mathrm{O} \leftrightarrow \mathrm{CO}_{2}+H_{2} & \Delta H=-41.1 \mathrm{~kJ} / \mathrm{mol}
\end{aligned}
$$

The entalphies of the reactions are given at $298 \mathrm{~K}$. Since a steam reforming unit consumes more energy than it produces, heat must be supplied to the unit in order to keep the temperature within the desired range. Another possible way of reforming methane is to make use of the partial oxidation reaction (POX) [1]:

$$
\mathrm{CH}_{4}+\frac{1}{2} \mathrm{O}_{2} \leftrightarrow \mathrm{CO}+2 \mathrm{H}_{2} \quad \Delta H=-36 \mathrm{~kJ} / \mathrm{mol}
$$

The partial oxidation process produces more energy than it consumes and does not require any external heat to be supplied. A typical POX-reformer is operating in a temperature range of $1200-1500^{\circ} \mathrm{C}$. The operating temperature can be reduced if a catalyst is added to the reactor. Such a process is known as catalytic partial oxidation (CPO) and is more suitible for small scale reforming than the high temperature process. One major disadvantage with POX and CPO reformers is that the amount of hydrogen produced per mole inlet methane is less than the corresponing amount for a steam reformer. Another disadvantage is that air has to be added to the gas mixture. This leads to a reduced partial pressure of hydrogen which in turn leads to lower system efficiency due to lower Nernst potentials in the cells [1].

ATR units combines the steam reforming reactions and partial oxidation so that no heat has to be supplied or removed from the reactor. This gives the autothermal reformer a higher thermal efficiency than those using regular steam reforming or partial oxidation [1]. The ATR unit also has some other interesting advantages compared to other reformers when considering start-up and unit size. In addition to the reforming reactions (35) - (38), other reactions will occur in the autothermal reformer as well $[11,20]$ :

$$
\begin{array}{rc}
\mathrm{CH}_{4}+2 \mathrm{O}_{2} \Leftrightarrow \mathrm{CO}_{2}+2 \mathrm{H}_{2} \mathrm{O} & \Delta H=-802.7 \mathrm{~kJ} / \mathrm{mol} \\
\mathrm{CH}_{4}+\mathrm{O}_{2} \Leftrightarrow \mathrm{CO}_{2}+2 \mathrm{H}_{2} & \Delta H=-71 \mathrm{~kJ} / \mathrm{mol} \\
\mathrm{CH}_{4}+\mathrm{CO}_{2} \Leftrightarrow 2 \mathrm{CO}+2 \mathrm{H}_{2} & \Delta H=247 \mathrm{~kJ} / \mathrm{mol} \\
2 \mathrm{CO} \Leftrightarrow \mathrm{C}+\mathrm{CO}_{2} & \Delta H=-172 \mathrm{~kJ} / \mathrm{mol} \\
C \mathrm{CH}_{4} \Leftrightarrow C+2 \mathrm{H}_{2} & \Delta H=75 \mathrm{~kJ} / \mathrm{mol}
\end{array}
$$


Results from literature [20] have been used to calculate the reaction rates of the reactions in the ATR unit. Only the most significant reaction rates are calculated in this article in order to reduce the number of calculations. Therefore only the steam reforming reactions $(35),(36)$, the water-gas shift reaction (37) and the total combustion reaction (39) is modeled. The reaction rates are calculated as:

$$
\begin{array}{r}
R_{1}=\frac{k_{1}}{p_{H_{2}}^{2.5}}\left(p_{C H_{4}} p_{H_{2} O}-\frac{p_{H_{2}}^{3} p_{C O}}{K_{I}}\right) \times \frac{1}{\Omega^{2}} \\
R_{2}=\frac{k_{2}}{p_{H_{2}}^{3.5}}\left(p_{C H_{4}} p_{H_{2} O}^{2}-\frac{p_{H_{2}}^{4} p_{C O_{2}}}{K_{I I}}\right) \times \frac{1}{\Omega^{2}} \\
R_{3}=\frac{k_{3}}{p_{H_{2}}}\left(p_{C O} p_{H_{2} O}-\frac{p_{H_{2}} p_{C O_{2}}}{K_{I I I}}\right) \times \frac{1}{\Omega^{2}} \\
R_{4}=\frac{k_{4 b} p_{C H_{4}} p_{O_{2}}}{\left(1+K_{C H_{4}}^{C} p_{C H_{4}}+K_{O_{2}}^{C} p_{O_{2}}\right)^{2}}+\frac{k_{C H_{4}} p_{O_{2}}}{1+K_{C H_{4}}^{C} p_{C H_{4}}+K_{O_{2}}^{C} p_{O_{2}}} \\
\Omega=1+K_{C O} p_{C O}+K_{H_{2}} p_{H_{2}}+K_{C H_{4}} p_{C H_{4}}+K_{H_{2} O} \frac{p_{H_{2} O}}{P_{H_{2}}}
\end{array}
$$

Here $R_{1}, R_{2}, R_{3}$ and $R_{4}$ are the corresponding reaction rates to reactions (35), (36), (37) and (39), respectively. $k_{j}$ is the Arrhenius reaction constant for reactions $j=1, \ldots, 4$ and is calculated using the parameters given in Table 10 according to

$$
k_{j}=k_{o j} \times \exp \left(\frac{-E_{j}}{R T}\right)
$$

$K_{j}$ is the equilibrium constant for reaction $j$ and is listed in Table 11 . The Van't Hoff species adsorption constants $K_{i}$ and $K_{i}^{C}$ for species $i$ are calculated in a similar way according to

$$
\begin{gathered}
K_{i}=K_{o i} \times \exp \left(\frac{-\triangle H_{i}}{R T}\right) \\
K_{i}^{C}=K_{o i}^{C} \times \exp \left(\frac{-\triangle H_{i}^{C}}{R T}\right)
\end{gathered}
$$

using the parameters given in Table 12 . 


\begin{tabular}{lcc} 
Reaction,$j$ & $k_{o j}(\mathrm{~mol} /(\mathrm{kg}$ cat $\mathrm{s}))$ & $E_{j}(\mathrm{~J} / \mathrm{mol})$ \\
\hline 1 & $1.17 \times 10^{15} \mathrm{bar}^{0.5}$ & 240100 \\
2 & $2.83 \times 10^{14} \mathrm{bar}^{0.5}$ & 243900 \\
3 & $5.43 \times 10^{5} \mathrm{bar}^{-1}$ & 67130 \\
$4 \mathrm{a}$ & $8.11 \times 10^{5} \mathrm{bar}^{-2}$ & 86000 \\
$4 \mathrm{~b}$ & $6.82 \times 10^{5} \mathrm{bar}^{-2}$ & 86000
\end{tabular}

Table 10: Arrhenius kinetic parameters

\begin{tabular}{lr} 
Reaction, $\mathrm{j}$ & Equilibrium constant, $K_{j}$ \\
\hline 1 & $K_{I}=\exp \left(\frac{-26830}{T_{s}}+30.114\right)\left(\mathrm{bar}^{2}\right)$ \\
2 & $K_{I I}=K_{I} \cdot K_{I I I}\left(\mathrm{bar}^{2}\right)$ \\
3 & $K_{I I I}=\exp \left(\frac{4400}{T_{s}}-4.036\right)$
\end{tabular}

Table 11: Reaction equilibrium constants

\begin{tabular}{llrrr} 
Substance, $i$ & $K_{o i}\left(\mathrm{bar}^{-1}\right)$ & $\triangle H_{i}(\mathrm{~J} / \mathrm{mol})$ & $K_{o i}^{C}\left(\mathrm{bar}^{-1}\right)$ & $\Delta H_{i}^{C}(\mathrm{~J} / \mathrm{mol})$ \\
\hline $\mathrm{CH}_{4}$ & $6.65 \times 10^{-4}$ & -38280 & & \\
$\mathrm{CO}$ & $8.23 \times 10^{-5}$ & -70650 & & \\
$\mathrm{H}_{2}$ & $6.12 \times 10^{-9}$ & -82900 & & \\
$\mathrm{H}_{2} \mathrm{O}$ & $1.77 \times 10^{5} \mathrm{bar}$ & 88680 & & \\
$\mathrm{CH}_{4}$ (combustion) & & & $1.26 \times 10^{-1}$ & -27300 \\
$\mathrm{O}_{2}$ (combustion) & & $7.78 \times 10^{-7}$ & -92800
\end{tabular}

Table 12: Van't Hoff parameters for species adsorption

The determined reaction rates are used for calculating the rate of formation of each substance. This is done according to:

$$
\begin{array}{r}
r_{C H_{4}}=-\eta_{1} R_{1}-\eta_{2} R_{2}-\eta_{4} R_{4} \\
r_{O_{2}}=-2 \eta_{4} R_{4} \\
r_{C O_{2}}=\eta_{2} R_{2}+\eta_{3} R_{3}+\eta_{4} R_{4} \\
r_{H_{2} O}=-\eta_{1} R_{1}-2 \eta_{2} R_{2}-\eta_{3} R_{3}-2 \eta_{4} R_{4} \\
r_{H_{2}}=3 \eta_{1} R_{1}+4 \eta_{2} R_{2}+\eta_{3} R_{3} \\
r_{C O}=\eta_{1} R_{1}-\eta_{3} R_{3}
\end{array}
$$

The $\eta$ factors are effectiveness factors for the reaction and are set to: $\eta_{1}=$ $0.07, \eta_{2}=0.06, \eta_{3}=0.7$ and $\eta_{4}=0.05$ [20]. 


\subsubsection{Implementation}

The implementation of the autothermal reformer model consists of a mixing volume with reforming reactions, shown in Figure 12. Fuel and air is fed to the volume through two different stream connectors. The air properties (mass fractions and enthalpy) are added to the fuel using a component mixing vector. In order for this to work, the fuel medium must be the ReformateFuel medium previously described in section 3.1.1. The reformed fuel leaves the volume through the stream connector to the left in Figure 12. External heating or cooling can be added to the reformer using the heat connector on the top of the volume.

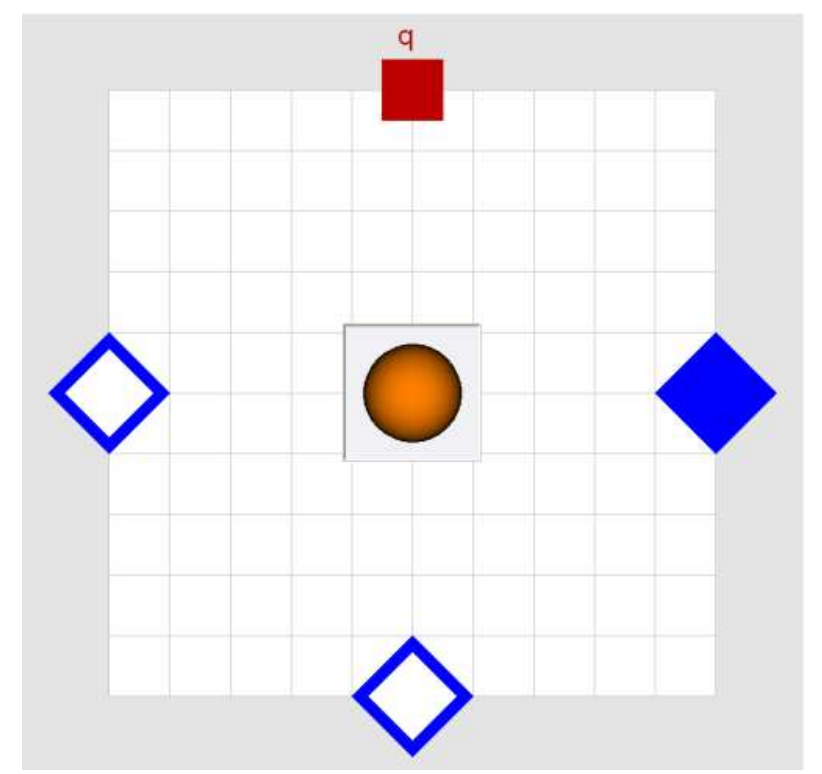

Figure 12: Graphical layout of the autothermal reformer

Reactions in the reformer are modeled by creating a DynamicAutoReformer reaction object. The DynamicAutoReformer model consists of equations calculating the reaction rates as described by equations (44) - (46). The determined reaction rates are then used for calculation of the rate of formation of each substance, described by (52) - (57). Component balance equations in the reaction model are similar to the ones previously used in the substack reactions, but some changes have been made in order to make the model compatible with the reaction rate equations (44) - (46).

The heat capacity in the above model is low since it does not yet include any solid material. This leads to unrealistically fast changes of the gas temperature. More realistic temperature changes is obtained by including a thermal mass representing the $\mathrm{Ni} / \mathrm{Al}_{2} \mathrm{O}_{3}$ housing often used for the reformer. This is included in the model in Figure 13. Here, thermal mass is included by connecting a metal body to the heat port of the autothermal reformer model.

Some expressions for evaluation of the reformer performance have been im- 


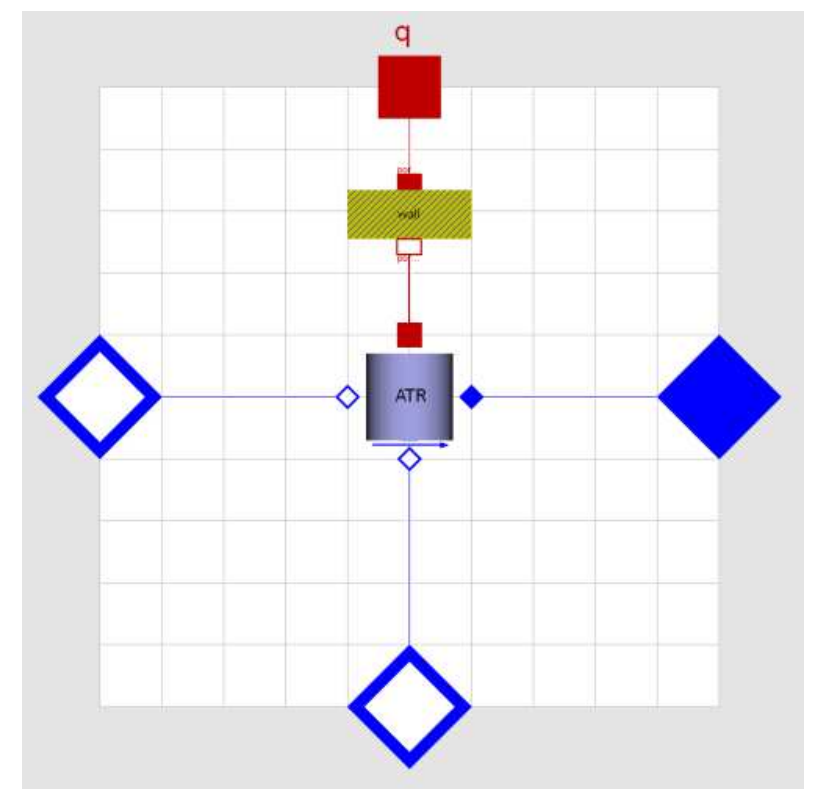

Figure 13: Graphical layout of the reformer unit

plemented in the ATR model. Utilization of $\mathrm{CH}_{4}$ and $\mathrm{O}_{2}$ are two key figures. Utilizaton shows how much of the available substance that has been used by the reformer. A utilization of 1 means that all available substance has been used and 0 means that nothing has been used.

The steam carbon ratio $(\mathrm{S} / \mathrm{C})$ and the oxygen gas carbon ratio $(\mathrm{O} / \mathrm{C})$ are two important key figures describing the inlet composition to the reformer. A steam carbon ratio is also calculated for the outlet gas. This is interesting as the outlet gas from the reformer is the inlet gas to the fuel cell stack. This is also the main reason why dry mole fractions in the outlet gas are calculated.

The thermal efficiency of the reformer is determined using an efficiency measure [20]:

$$
\eta_{\text {eff }}=\frac{n_{\mathrm{H}_{2}}}{n_{C H_{4}}} \cdot \frac{L H V_{\mathrm{H}_{2}}}{L H V_{C H_{4}}}
$$

Here $n_{H_{2}} / n_{\mathrm{CH}_{4}}$ is the number of mole hydrogen produced per mole methane fed to the reformer. $L H V_{H_{2}}$ and $L H V_{C_{H_{4}}}$ are the lower heating values of hydrogen gas and methane. 


\subsection{Catalytic burner}

When the fuel has passed through the SOFC stack it may still contain some unused hydrogen, carbon monoxide and methane. To recover the energy still available in these components and to reduce emissions the exhaust gas is often fed to a catalytic burner, where it is burned together with part of the exhaust gas from the cathode or fresh air. The produced heat can be used to generate steam for the pre-reformer, to pre-heat the natural gas, or for combined heat and power applications. In tests of catalytic afterburners full conversion of hydrogen and carbon monoxide have been achieved already at $400^{\circ} \mathrm{C}$, and full conversion of methane has been achieved at $700^{\circ} \mathrm{C}$. Thus SOFC plants can be expected to operate with very low emission levels [19].

For this purpose a model of a catalytic burner including reactions for oxidation of methane, carbon monoxide and hydrogen is implemented [11]:

$$
\begin{array}{r}
\mathrm{CH}_{4}+2 \mathrm{O}_{2} \rightarrow \mathrm{CO}_{2}+2 \mathrm{H}_{2} \mathrm{O} \\
\mathrm{CO}+\frac{1}{2} \mathrm{O}_{2} \rightarrow \mathrm{CO}_{2} \\
\mathrm{H}_{2}+\frac{1}{2} \mathrm{O}_{2} \rightarrow \mathrm{H}_{2} \mathrm{O}
\end{array}
$$

The implementation is based on a previous implementation made at Modelon. It is based on a volume model with air and fuel inlet ports and an air outlet port. It also has a port for heat flow to the environment and a boolean input for ignition signals. The burner operates in one of two states, either burning or mixing, where the difference is the stoichiometry matrix defining how the fuel components is mixed into the air flow. In the burning case the following stoichiometry matrix is used:

$$
S_{\text {Burning }}=\left(\begin{array}{ccccccc}
0 & 0 & 0 & 0 & 0 & 0 & 0 \\
0 & 1 & 1 & 1 & 0 & 0 & 0 \\
1 & 2 & 0 & 0 & 1 & 0 & 0 \\
0 & 0 & 0 & 0 & 0 & 1 & 0 \\
-0.5 & -2 & -0.5 & 0 & 0 & 0 & 1
\end{array}\right)
$$

The first 3 columns defines the reactions (59) to (61) and the remaining part defines how the $\mathrm{CO}_{2}, \mathrm{H}_{2} \mathrm{O}, \mathrm{N}_{2}$ and $\mathrm{O}_{2}$ components are moved from the fuel to the air model. In the non-burning case the stoichiometry matrix below is used. It excludes the reactions and only defines how to move components from the fuel to the air. In this case inflow of fuel components not included in the air model is simply dropped from the model.

$$
S_{\text {Mixing }}=\left(\begin{array}{ccccccc}
0 & 0 & 0 & 0 & 0 & 0 & 0 \\
0 & 0 & 0 & 1 & 0 & 0 & 0 \\
0 & 0 & 0 & 0 & 1 & 0 & 0 \\
0 & 0 & 0 & 0 & 0 & 1 & 0 \\
0 & 0 & 0 & 0 & 0 & 0 & 1
\end{array}\right)
$$

The components in the fuel model are, ordered after index, $\mathrm{H}_{2}, \mathrm{CH}_{4}, \mathrm{CO}$, $\mathrm{CO}_{2}, \mathrm{H}_{2} \mathrm{O}, \mathrm{N}_{2}, \mathrm{O}_{2}$ and the components in the air model are $\mathrm{Ar}, \mathrm{CO}_{2}, \mathrm{H}_{2} \mathrm{O}$, 
$N_{2}, O_{2}$. In the burner model an air/fuel ratio $\lambda$ is defined as

$$
\lambda=\frac{O 2_{\text {flow,air }}}{-S_{\text {Burning }, O_{2}} \cdot z_{\text {flow }, \text { fuel }}}
$$

where $O 2_{\text {flow, air }}$ is the molar inlet flow of $\mathrm{O}_{2}$ at the air inlet port, $S_{\text {Burning, } O_{2}}$ is the row in the $S_{\text {Burning }}$ matrix corresponding to production of $O_{2}$ in the air during reaction and $z_{\text {flow, fuel }}$ is a vector containing the molar flow of every fuel component at the fuel inlet port. With this expression $\lambda$ is the ratio of oxygen inlet flow versus the oxygen flow required for complete combustion of the current fuel inlet flow.

Using the $\lambda$ parameter the mode of operation is determined. If $\lambda$ is below a certain value and the ignition signal is set to true the burner enters the burning state. If the burner is operating in the burning state and $\lambda$ increases above a maximum value the combustion is stopped by setting the burner to the mixing state.

When combustion occurs a combustion rate defined as the fraction of fuel burned, $k_{\text {rate }}$, is calculated dynamically according to:

$$
\dot{k}_{\text {rate }}=\lambda-5 \cdot X i_{O 2}-k_{\text {rate }}
$$

where $\mathrm{Xi}_{\mathrm{O} 2}$ is the $\mathrm{O}_{2}$ molar fraction in the burner. With this definition of the combustion rate the energy and mass balances of the volume can be written as

$$
\begin{array}{r}
\dot{U}=m_{\text {flow, airinlet }} \cdot H_{\text {airinlet }}+m_{\text {flow, fuelinlet }} \cdot H_{\text {fuelinlet }}- \\
-m_{\text {flow, airoutlet }} \cdot H_{\text {gas }}+Q \\
\dot{m}=m_{\text {flow, airinlet }} \cdot X i_{\text {airinlet }}+S \cdot m_{\text {flow }, \text { fuelinlet }} \cdot X i_{\text {fuelinlet }} \cdot k_{\text {rate }}- \\
-m_{\text {flow, airoutlet }} \cdot X i_{\text {gas }}
\end{array}
$$

where $\dot{U}$ is the time derivative of the internal energy, $H$ is the specific enthalpy, $Q$ is the supplied heat, $m$ is a vector with masses for each component in the volume, $X i$ is a vector containing the mass fractions of every component and $S$ is the stoichiometry matrix $S_{\text {Burning }}$ or $S_{\text {Mixing }}$ depending on the operating conditions.

The heat released due to combustion is calculated as

$$
\begin{gathered}
Q_{\text {combustion }}=H_{\text {formation }} \cdot m_{\text {flow }, \text { fuelinlet }} \cdot X i_{\text {fuelinlet }}- \\
-H_{\text {formation }} \cdot S_{\text {Burning }} \cdot m_{\text {flow }, \text { fuelinlet }} \cdot X i_{\text {fuelinlet }} \cdot k_{\text {rate }}
\end{gathered}
$$




\subsection{Other components}

\subsubsection{Steam generator}

For the autothermal reformer to work the natural gas must be mixed with steam to increase the water content and temperature. The objective is to use the waste heat from the catalytic burner to generate steam from a water source at ambient temperature, and for this purpose a steam generator object is required.

Such a component has previously been implemented by Modelon and can be reused. The model consists of two flow channels with heat transfer based on log-mean temperature difference. One channel contains the conversion from liquid water to steam and therefore includes two medium models. The channel for the hot gas contains the moist air model used for the burner exhaust gas. For simpler usage in the system modeling a new steam generator object has been implemented by including these two channels and connecting them thermally.

\subsubsection{Methane and steam mixing volume}

After the steam has been generated it must be mixed with the natural gas. This is done in a volume model where energy and mass balances are met. However since two different medium models are involved a standard mixing volume can not be used. For this purpose a steam mixing volume is created by extending the compressible vector volume previously used in the stack model, and adding the stoichiometry matrix describing the shift from the steam medium model to the reformate medium model index.

\subsubsection{Heat exchangers}

In several places in the system exhaust gases are used to heat air and fuel flows prior to entering various components. Heat exchanger models from the Modelon CombiPlant library are used for this purpose. The heat exchangers have been modified in order to work with the medium models described in section 3.1.1. 


\subsection{System model implementation}

\subsubsection{Hotbox model implementation}

The system can be implemented by connecting the individual components as the desired system layout, but to get some structure in the graphical layer of the model a so-called hotbox model is created. This component includes the steam generator, the fuel mixing volume, the ATR unit and the heat exchangers used for pre-heating of the natural gas and the ATR air supply. The layout of the hotbox model is shown in Figure 14.

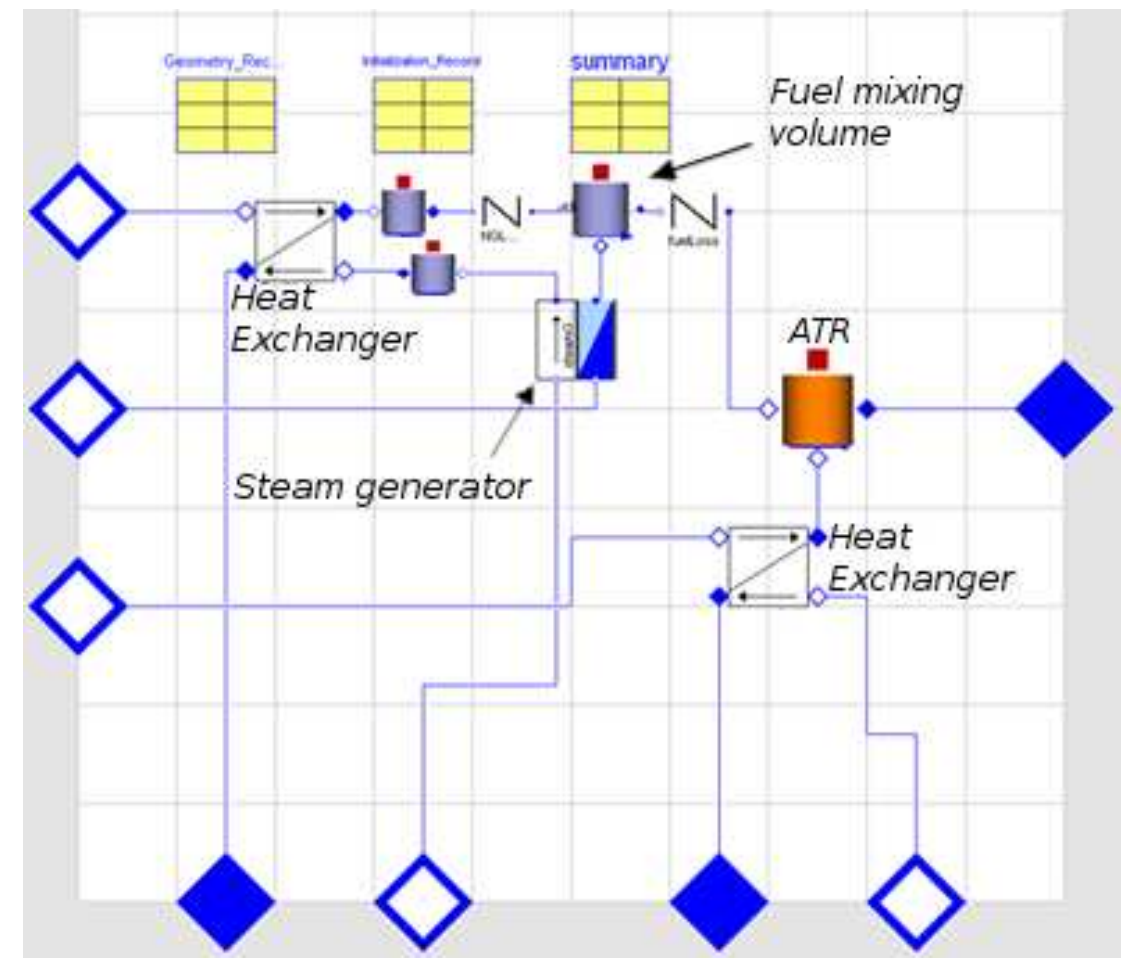

Figure 14: Graphical layout of the hotbox component

On the left side of the hotbox model three connectors are located. These are feed ports for natural gas to be supplied to the steam mixing volume, water to the steam generator and fresh air to the ATR unit, ordered from top to bottom. In the lower left side of the hotbox there are two connectors which are feed and drain ports for the hot exhaust gas from the catalytic burner. This gas is fed first to the steam generator, then to the heat exchanger heating the inlet natural gas and finally to the drain port. On the lower right side there are two connectors which are feed and drain ports for the anode off gas which is fed to the heat exchanger heating the air prior to entering the ATR unit. Finally on the right side there is the drain port for the reformed fuel.

There are also three records included in the hotbox model. These are used for setting geometry and initialization parameters of the included models directly 
as parameters of the hotbox model. There is also a summary record which reads the most important states from the different models and presents them in one place.

\subsubsection{Complete system implementation}

The system layout in Figure 11 can be achived by connecting the hotbox, stack and burner models to one another. The resulting system is shown in Figure 15.

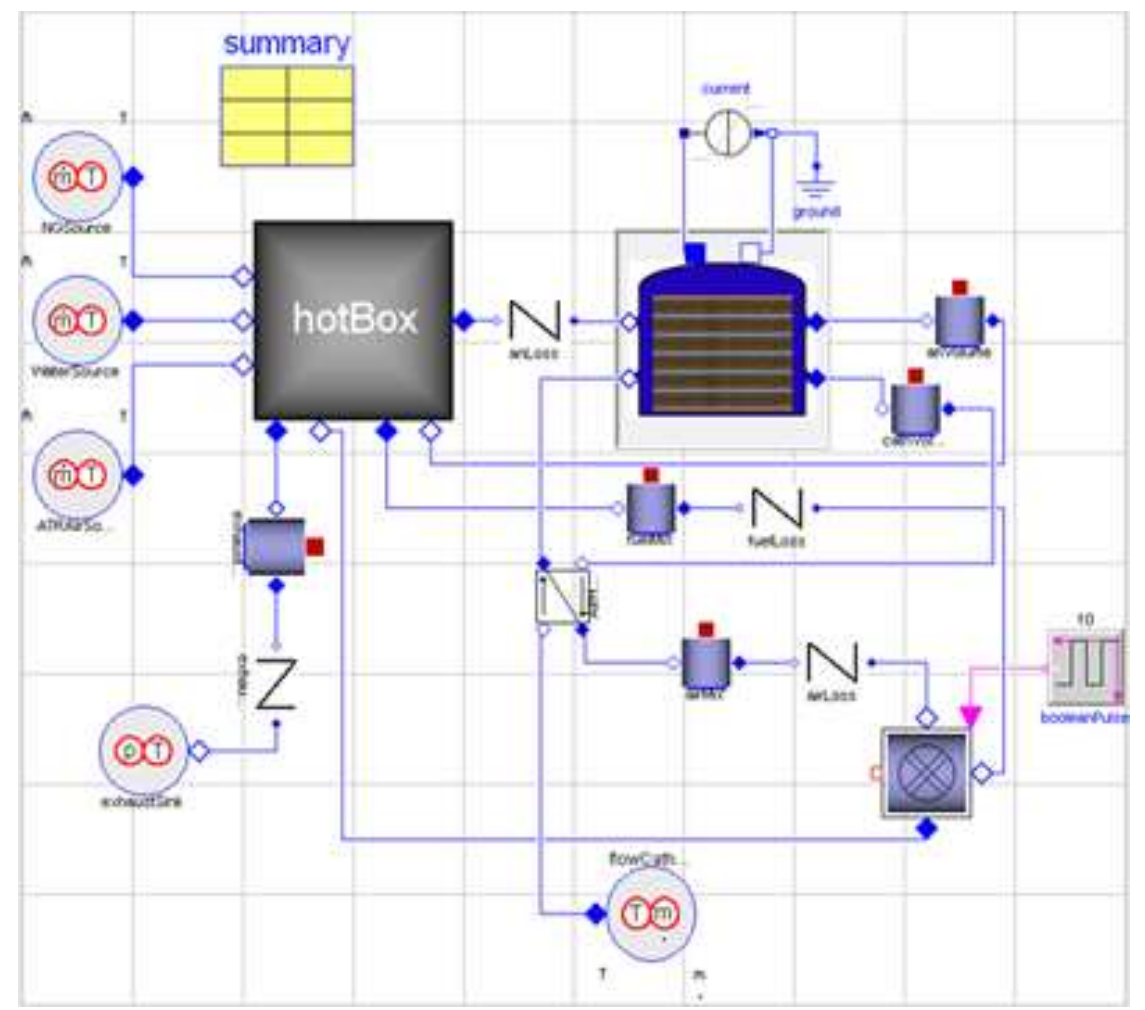

Figure 15: Graphical layout of the complete system

Natural gas, water and air are fed at a constant flow rate to the hotbox in order to mix and reform the fuel into a suitable composition. The reformed fuel from the hotbox is then fed as inlet fuel to the anode side of the stack. The exhaust gas from the stack anode is used to heat the hotbox inlet air before it enters the autothermal reformer and is then being burnt in the catalytic burner. Air is fed to the cathode side of the stack at a constant flow rate. Before it enters the stack it is heated in a heat exchanger by the hot outlet air from the stack cathode. The outlet air from the cathode is then connected to the burner. Exhaust gas from the burner is used in the hotbox in order to heat water in the steam generator and to heat natural gas at inlet. Small volumes and pressure drop models are added between some of the system components in order to give the system a more stable behaviour. The model also has a summary record that collects important system data during simulation and presents it in one place. 


\section{Results and Discussion}

This chapter covers model validation and presents results from simulations. First the cell models are simulated with constant fuel and temperature conditions and then the substack, stack and system models are simulated.

\subsection{Setup for investigation of electrical properties}

To investigate the behaviour of the electrochemical model defined in chapter 2.3 the model is simulated with a number of different geometries, fuel compositions and temperatures. To test the electrical properties the cell model is placed in a setup keeping the cell temperature and fuel and air compositions constant. The setup is shown in Figure 16.

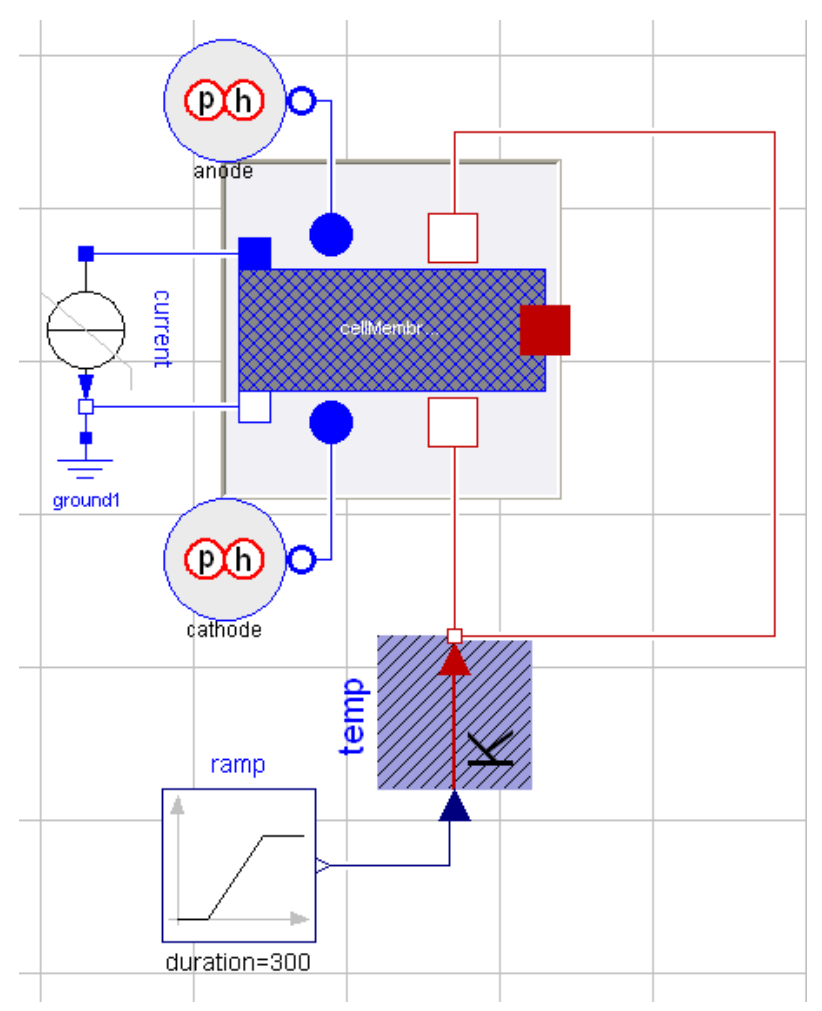

Figure 16: Setup for test of electrical properties

The cell model is positioned in the middle with a current generated between the electrical pins. There are sink objects on both the fuel and air sides, supplying a constant composition of fuel and air, respectively. To keep the temperature constant the heat capacity of the cell is set to a very large value and a constant temperature is set on the anode and cathode connectors. 


\subsection{Ohmic loss cell model}

In this simulation the behaviour of the ohmic loss model is investigated at constant temperature. The model parameters given in Table 13 are used and during the simulation the external current is ramped from $1 \mathrm{~A}$ to $150 \mathrm{~A}$.

\begin{tabular}{lr} 
Parameter & Value \\
\hline$A S R_{0}[11]$ & $0.29 \Omega \mathrm{cm}^{2}$ \\
$T_{0}[11]$ & $1073 \mathrm{~K}$ \\
$E_{a}[11]$ & $0.65 \mathrm{eV}$ \\
$A_{\text {cell }}[11]$ & $361 \mathrm{~cm}^{2}$ \\
$n_{\text {cell }}[11]$ & 50 \\
Fuel molar fraction [11] & $33 \% \mathrm{H}_{2}, 10 \% \mathrm{CH}_{4}, 6 \% \mathrm{CO}$ \\
& $6 \% \mathrm{CO}_{2}, 20 \% \mathrm{H}_{2} \mathrm{O}, 25 \% \mathrm{~N}_{2}$ \\
Air oxygen mass fraction & $23 \%$ \\
Stack temperature [11] & $760^{\circ} \mathrm{C}$
\end{tabular}

Table 13: Model parameters used in simulation

The simulation results are shown in Figure 17 as solid lines, together with simulation results from [11]. In those simulations a zero dimensional SOFC model is used and the limits are calculated by using the fuel compositions at the stack inlet and outlet, respectively. In the results from [11] the fuel was supplied from an autothermal reformer, where the oulet fuel molar fractions is flow rate dependent. In the simulation the flow rate has different values in the ranges 0 to $20 \mathrm{~A}, 20$ to $100 \mathrm{~A}$ and 100 to $150 \mathrm{~A}$. A constant fuel composition is used in the simulation in Figure 17. This is the same composition that is used from 20 to $100 \mathrm{~A}$ in [11].

In Figure 17(a) the result of this can be seen as the voltage is outside the limit for low currents. For larger currents the results are consistent, with only a small deviation which comes from the fact that in our simulation the stack inlet fuel composition is used at all times. In chapter 5.4.3 this cell model is simulated with a fuel flow including dynamic reactions. 
a

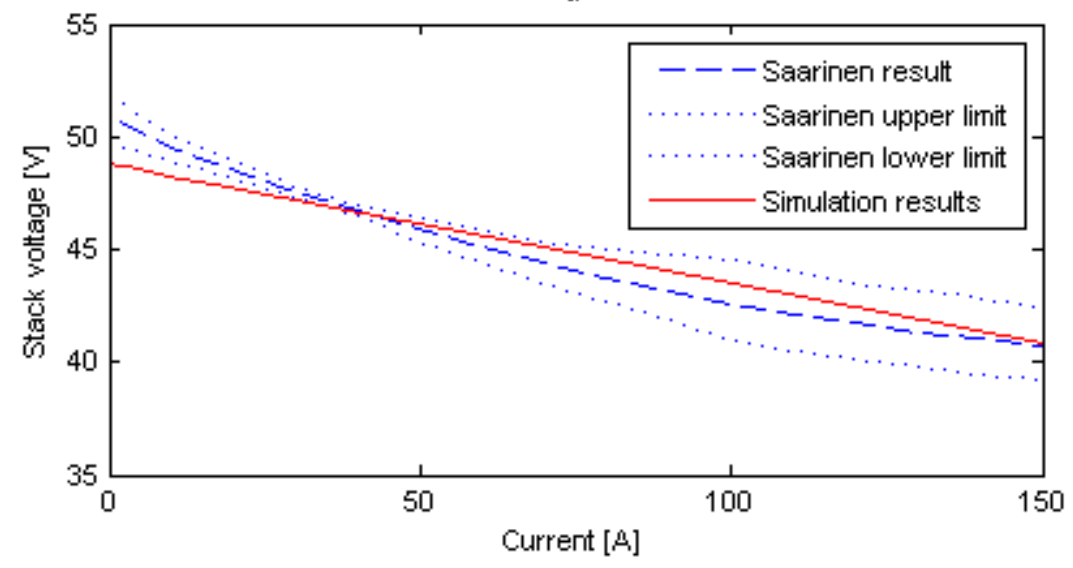

b

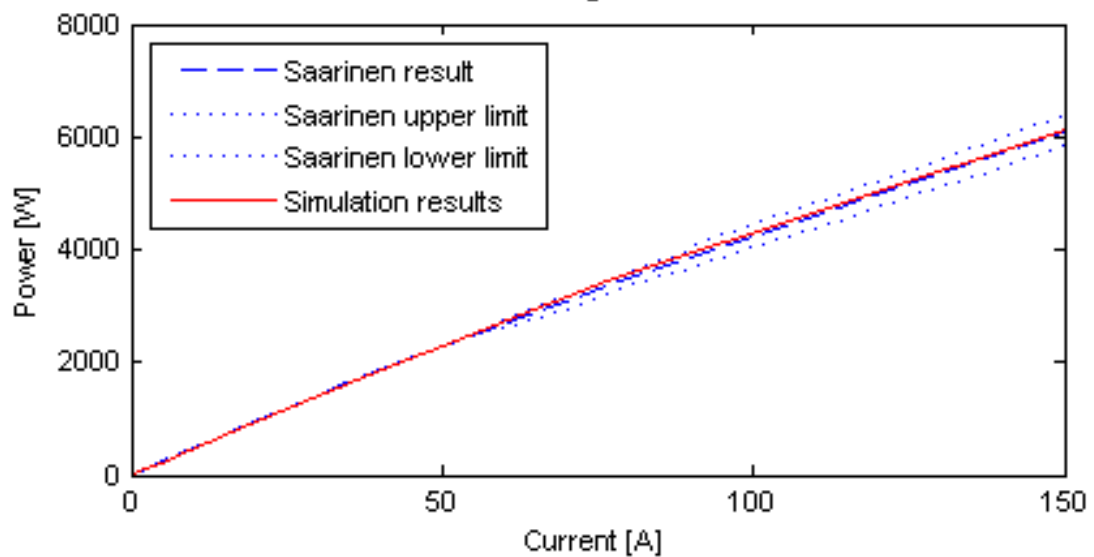

Figure 17: Simulation results and results from [11] for: (a) Stack voltage, (b) Generated power 


\subsection{Electrochemical cell model}

In this section the electrochemical cell model is investigated under constant temperature and fuel and air compositions. In all these simulations the model parameters and material properties given in [12], [16] and presented in Table 14 have been used.

\begin{tabular}{lrlr} 
Parameter & Value & $\mathbf{i} / \mathbf{j}$ & $D_{i, j}\left[\mathrm{~m}^{2} / \mathrm{s}\right]$ \\
\hline$\gamma_{a}$ & $5.5 \cdot 10^{8} \mathrm{~A} / \mathrm{m}^{2}$ & $C H_{4} / \mathrm{CO}$ & $3.47 \cdot 10^{-5}$ \\
$\gamma_{c}$ & $7 \cdot 10^{8} \mathrm{~A} / \mathrm{m}^{2}$ & $\mathrm{CH}_{4} / \mathrm{H}_{2} \mathrm{O}$ & $4.30 \cdot 10^{-5}$ \\
$E_{a c t, a}$ & $100 \cdot 10^{3} \mathrm{~J} / \mathrm{mol}$ & $\mathrm{CH}_{4} / \mathrm{H}_{2}$ & $11.04 \cdot 10^{-5}$ \\
$E_{a c t, c}$ & $120 \cdot 10^{3} \mathrm{~J} / \mathrm{mol}$ & $\mathrm{CH} H_{4} / \mathrm{CO}_{2}$ & $2.88 \cdot 10^{-5}$ \\
$\alpha_{a}$ & 0.5 & $\mathrm{CO} / \mathrm{H}_{2} \mathrm{O}$ & $4.15 \cdot 10^{-5}$ \\
$\alpha_{c}$ & 0.3 & $\mathrm{CO} / \mathrm{H}_{2}$ & $11.92 \cdot 10^{-5}$ \\
$\beta_{1, a}, \beta_{2, a}$ & $7800 \Omega^{-1} \mathrm{~m}^{-1}, 1290 \mathrm{~K}$ & $\mathrm{CO} / \mathrm{CO}_{2}$ & $2.59 \cdot 10^{-5}$ \\
$\beta_{1, e l}, \beta_{2, e l}$ & $20500 \Omega^{-1} \mathrm{~m}^{-1}, 9030 \mathrm{~K}$ & $\mathrm{H}_{2} \mathrm{O} / \mathrm{H}_{2}$ & $14.10 \cdot 10^{-5}$ \\
$\beta_{1, c}, \beta_{2, c}$ & $33770 \Omega^{-1} \mathrm{~m}^{-1}, 1130 \mathrm{~K}$ & $\mathrm{H}_{2} \mathrm{O} / \mathrm{CO}_{2}$ & $3.38 \cdot 10^{-5}$ \\
$\rho_{c c a}$ & $2 \cdot 10^{-4} \Omega m$ & $\mathrm{H}_{2} / \mathrm{CO}_{2}$ & $10.23 \cdot 10^{-5}$ \\
$\rho_{c c c}$ & $7.8 \cdot 10^{-5} \Omega m$ & & \\
$p_{r e f}$ & $1.0133 \cdot 10^{5} \mathrm{~Pa}$ & & \\
$D_{c}^{e f f}$ & $5.4 \cdot 10^{-6} \mathrm{~m}^{2} \mathrm{~s}^{-1}$ & &
\end{tabular}

Table 14: Model parameters and material properties

Also, unless anything else is given for a specific test, the cell geometry given in Table 15 has been used. The values represents typical geometries for electrolyte supported SOFCs presented in literature.

\begin{tabular}{lr} 
Parameter & Value \\
\hline Anode thickness & $20 \mu \mathrm{m}$ \\
Electrolyte thickness & $150 \mu \mathrm{m}$ \\
Cathode thickness & $20 \mu \mathrm{m}$ \\
Anode current collector thickness & $0.5 \mathrm{~mm}$ \\
Cathode current collector thickness & $20 \mu \mathrm{m}$ \\
Cell pitch length & $10 \mathrm{~mm}$ \\
Active cell area & $13.44 \mathrm{~cm}^{2}$
\end{tabular}

Table 15: Cell geometry used in simulations

Furthermore a fuel with molar fractions of 0.97 for $\mathrm{H}_{2}$ and 0.03 for $\mathrm{H}_{2} \mathrm{O}$ and air with 23 mass percent oxygen was used. The temperature was kept at $800^{\circ} \mathrm{C}$ and the initial stack pressure was 1.013 bar. The spliceValue parameter used for the linear activation loss approximation outside the Tafel region is set to $0.05 \mathrm{~V}$. 


\subsubsection{Material conductivities}

Firstly the material conductivities given by equation 18 and the parameters in Table 14 are evaluated at different temperatures. The results are given in Figure 18.
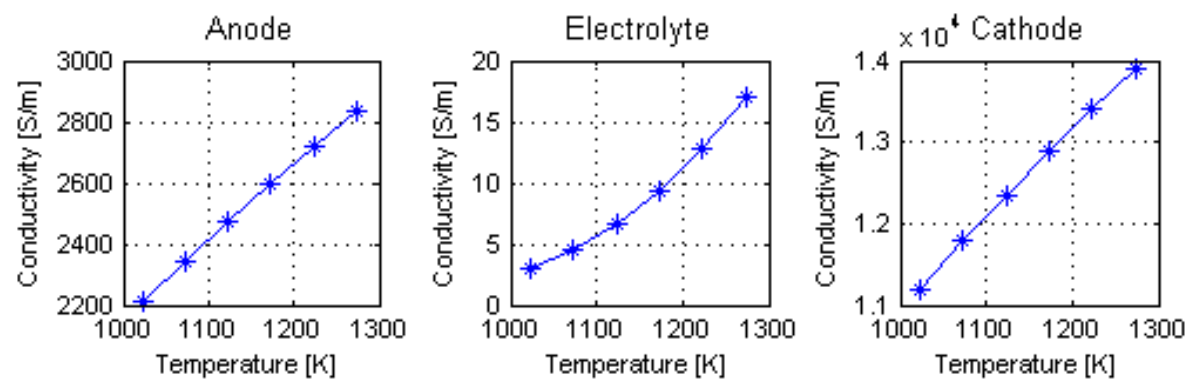

Figure 18: Material conductivities

For all materials the conductivity increases with increased temperature, and the values are also in the same order of magnitude as those given for typical SOFC materials in [13].

The conductivity of the electrolyte material is 2 orders of magnitude smaller than that of the anode and cathode materials. This is due to the fact that electricity is conducted in form of oxygen ions that diffuse through the electrolyte, whereas in the electrodes free electrons are conducted. The conductivity varies largely between different materials and production techniques used. For high temperature SOFCs zirconia doped with yttria is the most effective electrolyte material. Production techniques are improved continuously, increasing the conductivity and allowing for thinner electrolytes. The anode is often a composite of nickel and YSZ, where the zirconia gives a thermal expansion coefficien comparable to that of the electrolyte and the nickel acts as catalyst. For the cathode, however, strontium-doped lanthanum manganite, $\left(\mathrm{La}_{0.84} \mathrm{Sr}_{0.16}\right) \mathrm{MnO}_{3}$, is most commonly used and gives a conductivity slightly higher than that of the anode $[1,13]$.

\subsubsection{Activation loss approximation}

For the activation loss two different expressions are used depending on the cell operating condition. These are the Tafel equation (19), valid for currents greater than the exchange current density, and a linear approximation (20), valid for currents lower than the exchange current density. The behaviour in the merging of these two is investigated by making simulations with a current ramp that starts in the linear region and ends in the Tafel region. In the merging of the curves the result must not deviate too much from the original value, and it must be smooth so that simulations are not interrupted in the transition between the two regions.

Simulations are made at different temperatures to test the merging at both high and low exchange current densities. Results are shown in Figure 19. The 


\begin{tabular}{lrr} 
Temperature & $i_{0, a}\left[\mathrm{~A} / \mathrm{m}^{2}\right]$ & $i_{0, c}\left[\mathrm{~A} / \mathrm{m}^{2}\right]$ \\
\hline $700^{\circ} \mathrm{C}$ & 565.0 & 170.6 \\
$800^{\circ} \mathrm{C}$ & 1787.8 & 679.8 \\
$900^{\circ} \mathrm{C}$ & 4648.7 & 2139.8 \\
$1000^{\circ} \mathrm{C}$ & 10402 & 5625.2
\end{tabular}

Table 16: Exchange current densities at the temperatures in this simulation

exchange current densities for the different cases are given in Table 16 and it can be seen that they increase with increased temperature. Note that a different scale for the exchange current density is used in (a), (b) than in (c), (d). In all cases the Tafel equation and linear approximation are merged smoothly in a range around the intersection of the two curves. 


\begin{tabular}{|c|c|}
\hline$\longrightarrow$ Anode activation loss & Cathode activation loss \\
\hline--- Anode Tafel equation & --- Cathode Tafel equation \\
\hline ….... Anode linear approximation & . . . Cathode linear approximation \\
\hline
\end{tabular}

(a) $\mathrm{T}=700^{\circ} \mathrm{C}$

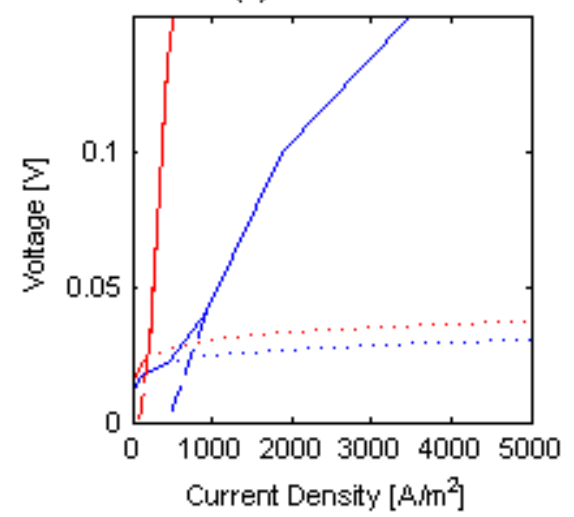

(b) $\mathrm{T}=800^{\circ} \mathrm{C}$

(c) $\mathrm{T}=900^{\circ} \mathrm{C}$
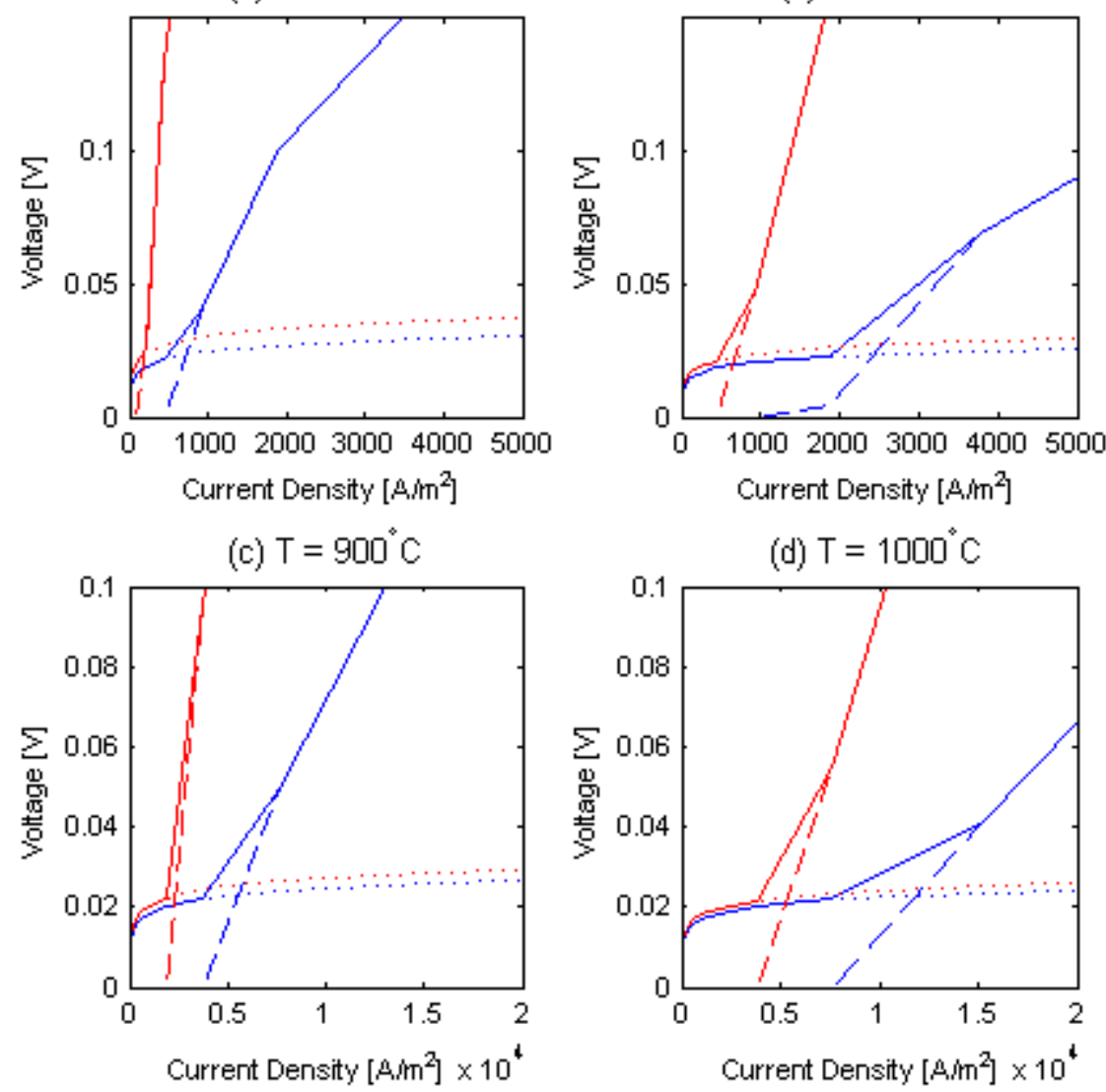

(d) $\mathrm{T}=1000^{\circ} \mathrm{C}$

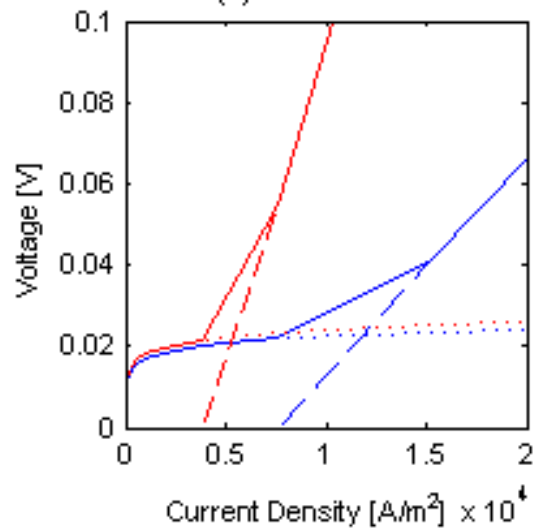

Figure 19: Anode and cathode activation losses versus current density at: (a) $700^{\circ} \mathrm{C}$, (b) $800^{\circ} \mathrm{C}$, (c) $900^{\circ} \mathrm{C}$, (d) $1000^{\circ} \mathrm{C}$. Also shown are the Tafel equations and linear approximations. 


\subsubsection{Cell pitch length dependency}

To investigate the ohmic loss for different cell pitch lengths a number of simulations are done. There will be no difference in fuel composition along the flow direction and therefore only one discretization is used. The number of cells in the stack is set to one to get per cell voltages.

The current through the external circuit is set to $1 \mathrm{~A}$ which corresponds to a current density of $744 \mathrm{~A} / \mathrm{m}^{2}$ through the cell. The activation and concentration losses are independent of the cell pitch length with values of $0.1601 \mathrm{~V}$ and $0.00104 \mathrm{~V}$, respectively. For the activation loss $0.114 \mathrm{~V}$ loss occurs at the anode side and $0.046 \mathrm{~V}$ loss occurs at the cathode side.

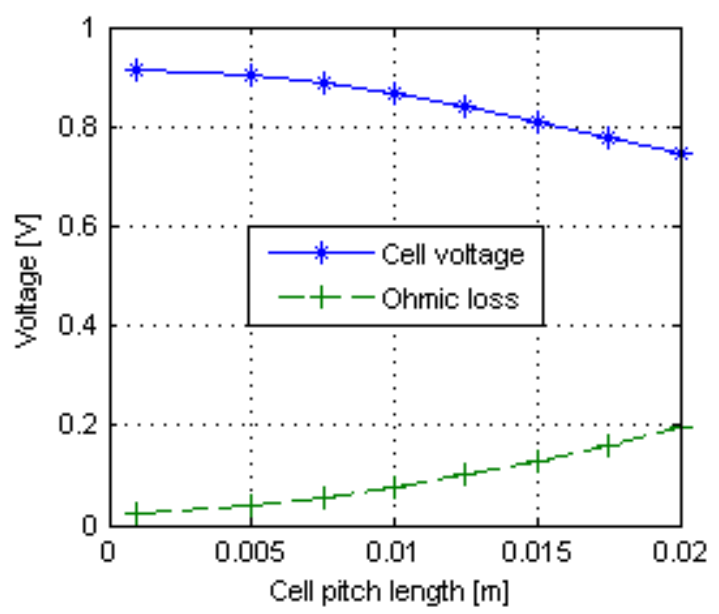

Figure 20: Cell voltage and ohmic loss versus cell pitch length

The resulting cell voltage and ohmic loss is shown in Figure 20. The total thickness of the cell layers with geometry according to Table 15 is $710 \mu \mathrm{m}$, making the cell cross-section close to quadratic in the simulation with a cell pitch length of $1 \mathrm{~mm}$. In this case the in-plane and cross-plane current paths are of the same order of magnitude. For increased cell pitch lengths the in-plane current path gets longer and thus contributes more to the total ohmic loss. With a $2 \mathrm{~cm}$ wide cell the ohmic loss has increased almost a factor 8 and the in-plane current now accounts for a dominant part of the ohmic loss. This may well be the case in real SOFCs of in-plane conducting configurations [13].

The ohmic loss curve also shows the same shape as given in the area specific resistance versus cell pitch length plot in [12], a perfect match in values has not been obtained however, because values for all geometry parameters are not given in that paper.

\subsubsection{Current collector thickness dependency}

Investigation of the effects of current collector thickness is conducted with the parameters and geometry defined in Tables 14 and 15. The activation loss is 
independent of the current collector thicknesses and is constant at $0.1601 \mathrm{~V}$. It is worth to note that only the anode current collector thickness was changed in this study. The resulting cell voltage is shown in Figure 21, together with the ohmic and concentration losses.
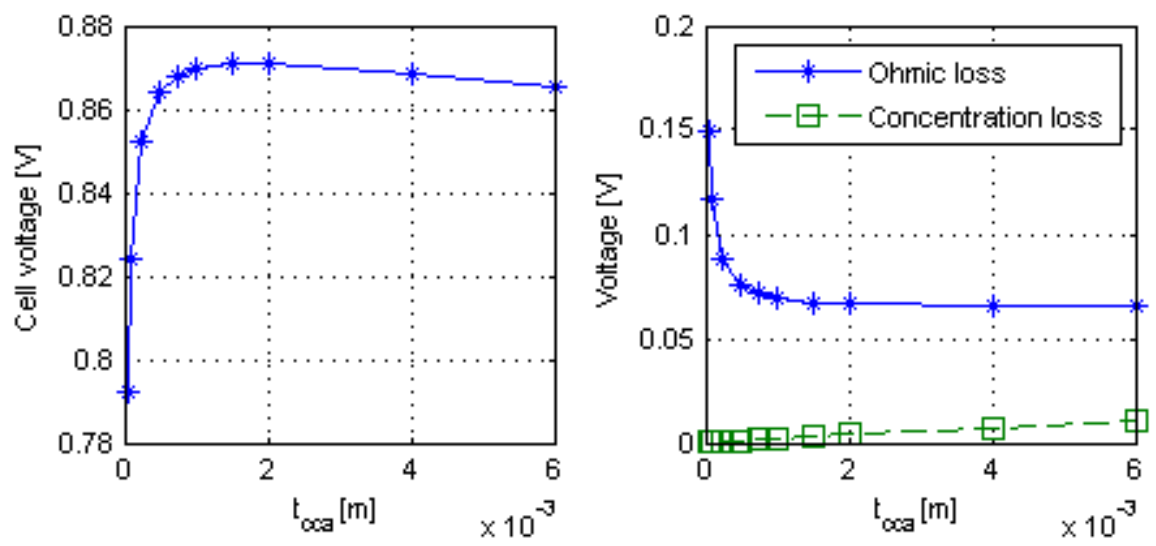

Figure 21: Cell voltage and losses versus anode current collector thickness

In these results two effects of varying the current collector thickness can be observed, the first being the large effect on the ohmic loss. In the thinnest case the thickness was set to $50 \mu \mathrm{m}$ giving a total cell thickness of $260 \mu \mathrm{m}$, which together with a cell pitch length of $10 \mathrm{~mm}$ gives a large loss from the in-plane current path. For a thickness larger than $2 \mathrm{~mm}$, corresponding to a total thickness larger than $2.21 \mathrm{~mm}$, the effect of in-plane currents is barely visible and the ohmic loss varies little when the thickness is increased further. In this case the electrolyte yields the dominant part of ohmic loss, as it has much lower conductivity than the current collector.

The second effect is the concentration loss which approximately increases linearly with increased current collector thickness. Even at the thickest case simulated here the concentration loss is much smaller than the ohmic loss. However, when summarizing the effects, the minimum loss, and thereby the maximum cell voltage is found at a current collector thickness of $2 \mathrm{~mm}$.

\subsubsection{Effects of varying current density}

The current density is an important aspect of the cell operating condition as it, directly or indirectly, affects all states in the model. The cell pitch length was set to $5 \mathrm{~mm}$ and otherwise the parameters and geometry in Tables 14 and 15 were used. The external current was varied from 1 to $10 \mathrm{~A}$ in steps of 1 A, giving current densities from 744 to $7440 \mathrm{~A} / \mathrm{m}^{2}$ in the cell. The simulation results for cell voltage and losses are shown in Figure 22.

In this case the activation loss turns out to be the dominant loss. This may well be the case for SOFCs at relatively low operating temperatures, as indicated in [12]. The temperature dependence of the losses is further investigated 


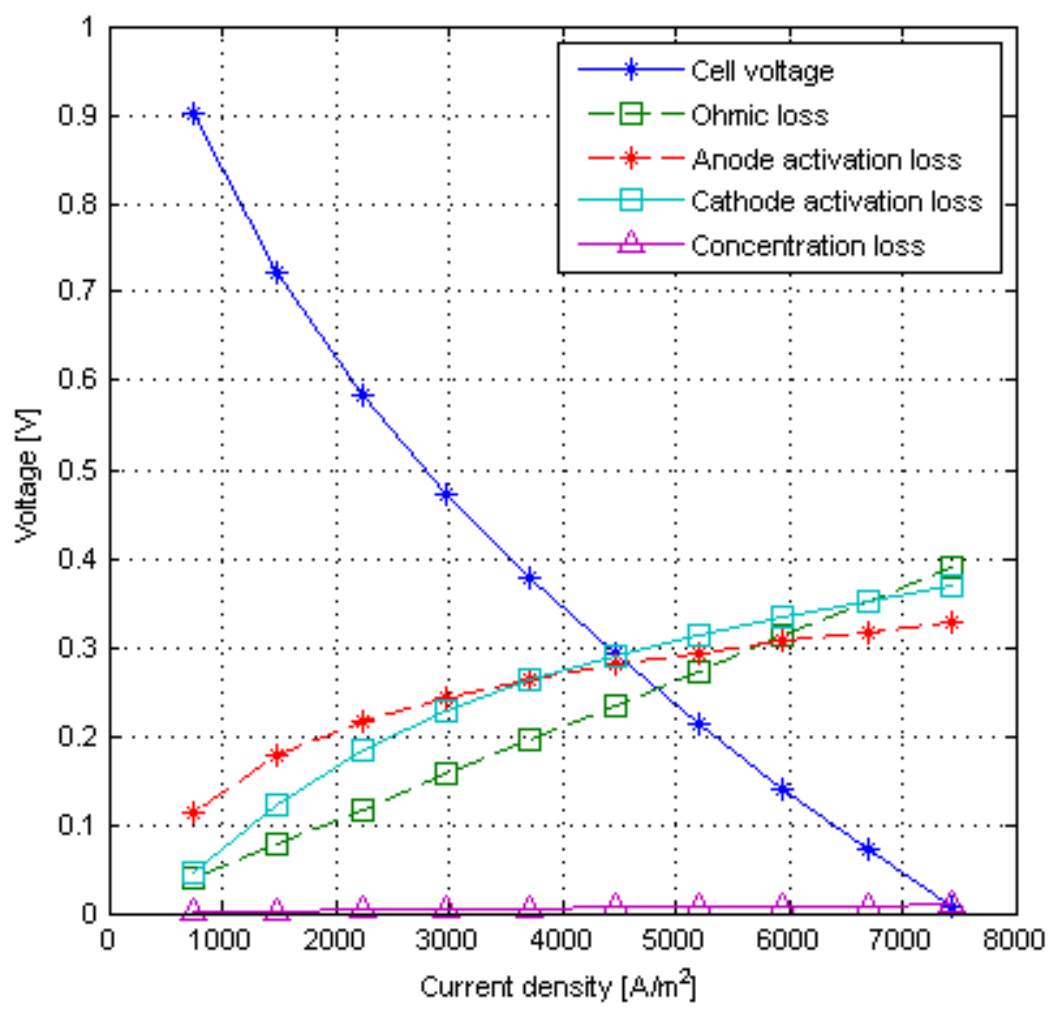

Figure 22: Cell voltage and losses versus current density

in chapter 5.3.6. At very low current densities the activation loss is dominant and also grows fastest with increased current density, as expected $[1,14]$.

At higher current densities the derivative of the activation loss is lower and the ohmic loss, which varies linearly with the current density at constant temperature, increases fastest, which is also expected [1]. The concentration loss is very small for all cases in this simulation and this is due to the fact that in this simulation the fuel composition is kept constant even though hydrogen is consumed. The dependence of the activation and concentration losses on fuel composition is investigated in chapter 5.3.7.

\subsubsection{Effects of varying temperature}

In this simulation the temperature dependence of the cell voltage and losses is investigated. The electrolyte thickness was set to $30 \mu \mathrm{m}$ and the cell pitch length was set to $5 \mathrm{~mm}$, the remaining parameters were set according to Tables 14 and 15. The current through the external circuit was set to $5 \mathrm{~A}$ which corresponds to an internal current density of $3720 \mathrm{~A} / \mathrm{m}^{2}$. The model is simulated at temperatures between $800^{\circ} \mathrm{C}$ and $1050^{\circ} \mathrm{C}$ in steps of $50^{\circ} \mathrm{C}$ and the results are shown in Figure 23. 


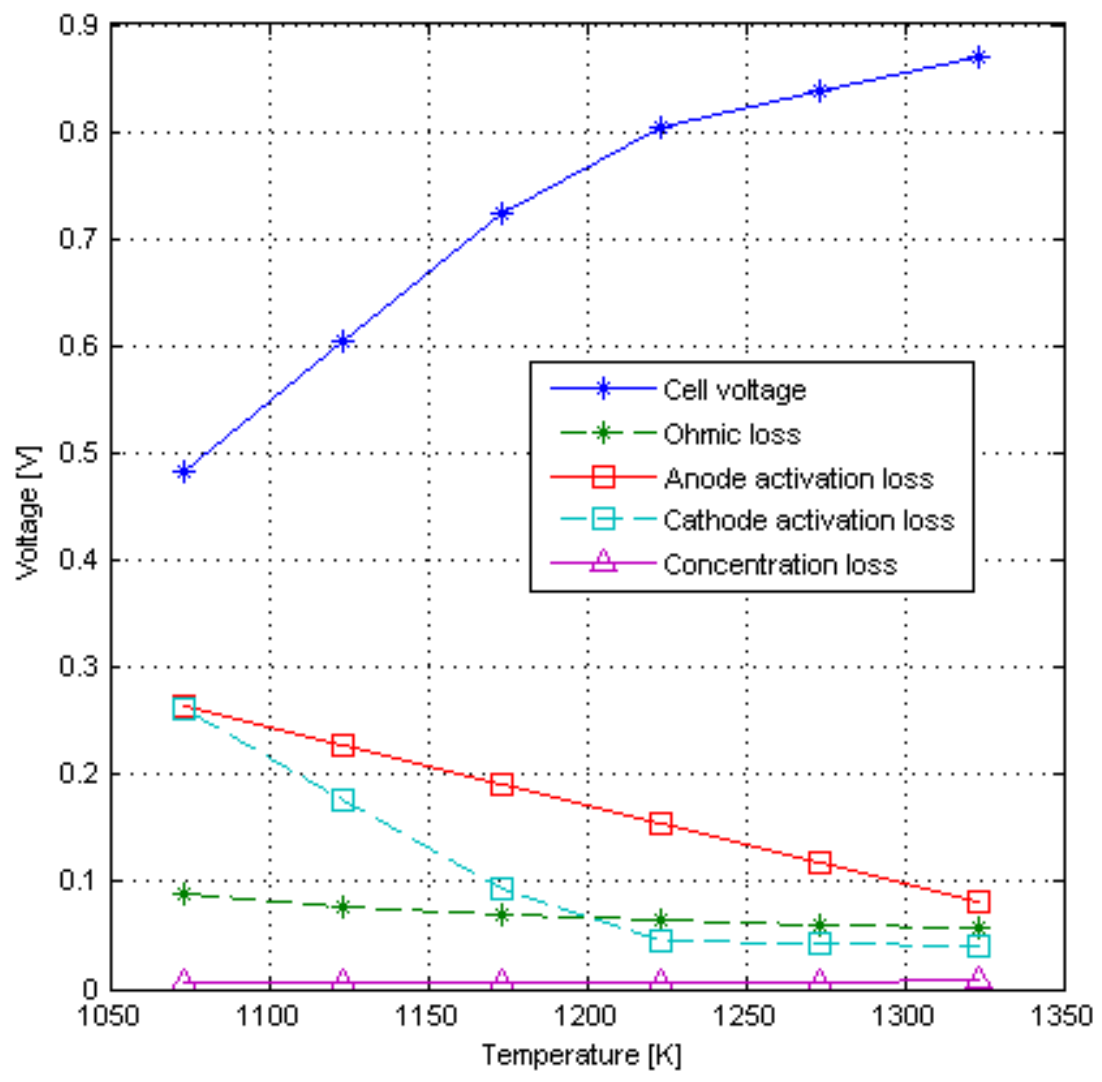

Figure 23: Cell voltage and losses versus temperature

The ohmic and activation losses, which are the dominant ones, both decrease with increased temperature and as a result the cell voltage shows a significant increase. At $950^{\circ} \mathrm{C}$ and above the activation loss at the cathode side is small and decreases little with further increased temperature and as a consequence the cell voltage increases more slowly above this temperature. The concentration loss is very small for all temperatures in this simulation because of the constant fuel composition. It increases slightly with increased temperature due to the temperature dependence of the mole fractions at the reaction sites (equations $25-27$ ), but the effect is very small. It has been hard to find measurements or validated simulation results for the temperature dependence of the individual losses in the literature, but the behaviour in the simulation is consistent with simulation results presented in [12].

\subsubsection{Effects of varying fuel composition}

In this section the effects of varying fuel composition is investigated. In these simulations the temperature was set to $850^{\circ} \mathrm{C}$, the electrolyte thickness was set to $30 \mu \mathrm{m}$ and the cell pitch length was set to $5 \mathrm{~mm}$. The remaning parameters were set according to Tables 14 and 15 . The spliceValue parameter was set to $0.025 \mathrm{~V}$ and the current through the external circuit was set to 5 
A which corresponds to an internal current density of $3720 \mathrm{~A} / \mathrm{m}^{2}$. The ohmic and cathode activation losses are independent of the fuel composition and were $0.0776 \mathrm{~V}$ and $0.1775 \mathrm{~V}$ respectively in all simulations. The simulation results for cell voltage, anode activation loss and concentration loss are shown in Figure 24.

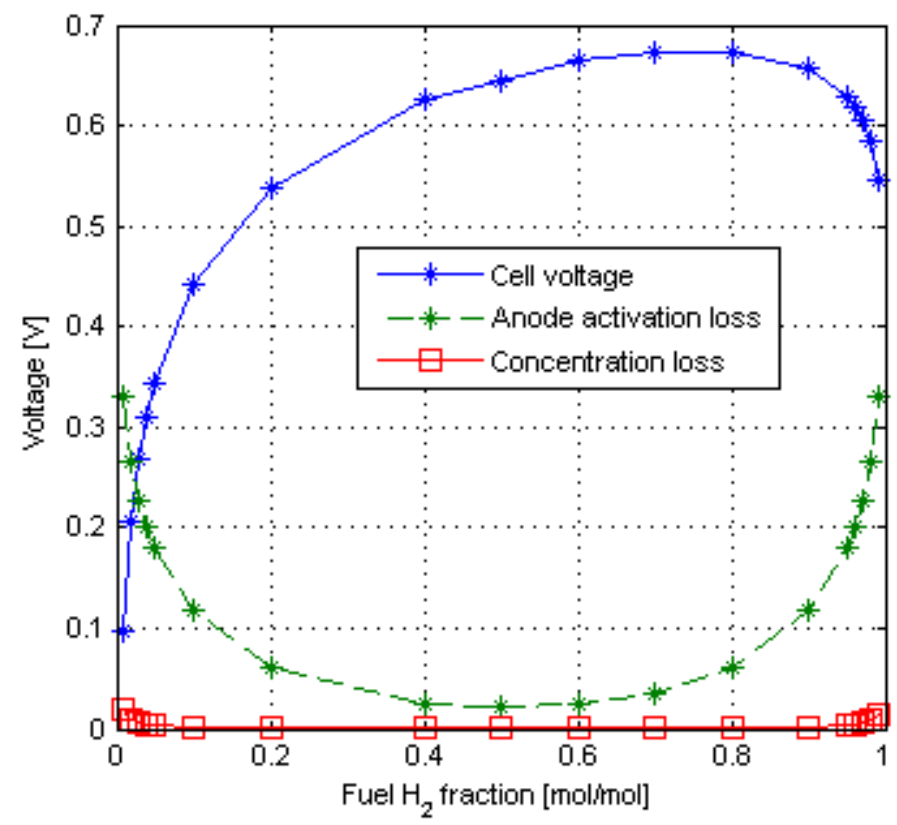

Figure 24: Cell voltage and losses versus fuel $\mathrm{H}_{2}$ molar fraction

Except for hydrogen molar fractions close to 0 or 1 the cell voltage increases with increased hydrogen fraction, as indicated by the Nernst equation (7). In the extreme cases however, the activation and concentration losses increases quickly and yields a quick decrease in the cell voltage. It is found that a maximum cell voltage appears at around $70 \%$ to $80 \%$ hydrogen content. The concentration loss is relatively low in all cases, but it also shows a large increase when the hydrogen fraction approaches 0 or 1 . For example, when the hydrogen fraction is decreased from $10 \%$ to $1 \%$ the concentration loss shows an $840 \%$ increase. The behaviour with minimum values in the range of $40 \%$ to $60 \%$ hydrogen is consistent with the results in [12], however the simulations give less concentration loss due to a higher diffusion coefficient and possibly a thinner anode. 


\subsection{Substack model validation}

\subsubsection{Setup for substack model validation}

For substack simulations the setup shown in Figure 25 has been used.

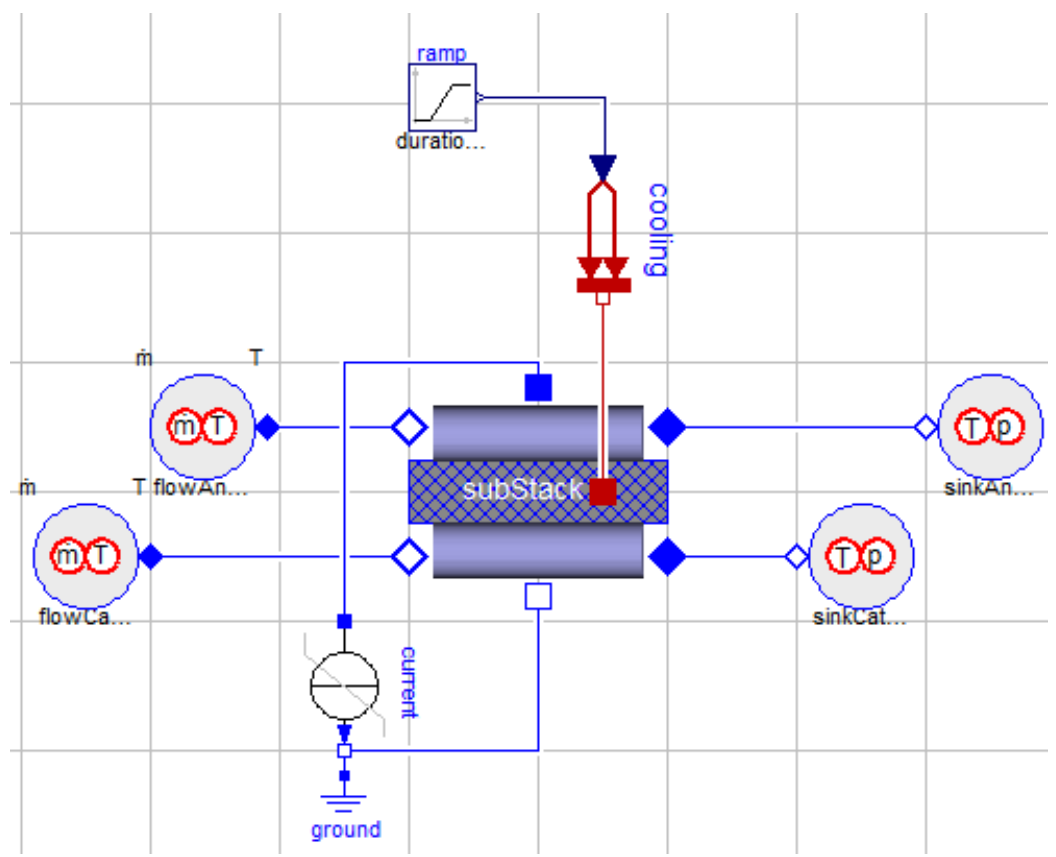

Figure 25: Experiment setup for substack validation

In the middle is the substack with a current generated between its electrical pins. Also connected to the substack is a heat generating block that can be used to cool or heat the stack by adding or removing heat energy directly at the cell model. Sinks are connected to the outlets of the stack and the purpose is to define a constant pressure at the stack outlet. There is also a medium model with a constant composition and temperature, that is used if there is a negative outflow from the stack in its transient behaviour. Connected at the stack inlets are source objects that contain medium models with constant composition and temperature. From the source object there is a constant outflow, independent of the pressure at the stack inlet.

\subsubsection{Effects of discretization}

The cell is modeled by discretized parts and the bulk flows are modeled by a finite volume method with the same number of elements as cell model discretizations. A discretization is an approximation causing a deviation of the model from the real process, and the fewer discretizations the larger the deviation. It is important to look at how the number of discretizations affects the simulation results. If the discretizations are implemented correctly a small change in the number of discretizations will not have a large impact on the results, and for an increasing number of discretizations the results should converge towards ones 
with negligible discretization error.

\begin{tabular}{lr} 
Parameter & Value \\
\hline Fuel inlet flow & $2.07 \cdot 10^{-6} \mathrm{~kg} / \mathrm{s}$ \\
Fuel inlet temperature & $900^{\circ} \mathrm{C}$ \\
Fuel inlet pressure & $1.013 \mathrm{bar}$ \\
Fuel $\mathrm{CH}_{4}$ mass fraction & $17.1 \%$ \\
Fuel $\mathrm{H}_{2}$ mass fraction & $26.3 \%$ \\
Fuel $\mathrm{H}_{2} \mathrm{O}$ mass fraction & $49.3 \%$ \\
Fuel $\mathrm{CO}$ mass fraction & $2.9 \%$ \\
Fuel $\mathrm{CO}$ mass fraction & $4.4 \%$ \\
Air inlet flow & $9.42 \cdot 10^{-5} \mathrm{~kg} / \mathrm{s}$ \\
Air inlet temperature & $900^{\circ} \mathrm{C}$ \\
Air inlet pressure & $1.013 \mathrm{bar}$ \\
Active cell area & $0.01 \mathrm{~m}^{2}$ \\
Number of cells in stack & 2 \\
Current through stack & $10 \mathrm{~A}$ \\
Bulk channel volumes & $0.001 \mathrm{~m}^{3}$ \\
Total cell heat capacity & $412 \mathrm{~J} / \mathrm{K}$
\end{tabular}

Table 17: Test case parameters for investigation of effects of discretization

By running a number of simulations under the same conditions, varying only the number of discretizations, possible errors in the implementation can be detected and a reasonable number of discretizations for other simulations can be determined. A test case defined in Table 17, where data for fuel flow and composition are from Case II in [2], and steady-state values for a number of parameters for different number of discretizations are presented in Table 18.

\begin{tabular}{lrrr}
$\mathbf{N}$ & Utilization & Stack voltage [V] & Heat generated [W] \\
\hline 1 & 0,697900 & 1,41910 & 15,0980 \\
2 & 0,773370 & 1,43310 & 15,0681 \\
3 & 0,805980 & 1,43323 & 15,1065 \\
4 & 0,822993 & 1,43297 & 15,1304 \\
5 & 0,833219 & 1,43285 & 15,1450 \\
6 & 0,840017 & 1,43280 & 15,1546 \\
7 & 0,845142 & 1,43234 & 15,1639 \\
8 & 0,849417 & 1,43133 & 15,1788 \\
9 & 0,852710 & 1,43068 & 15,1827 \\
10 & 0,855355 & 1,43023 & 15,1891 \\
& & & \\
20 & 0,869961 & 1,42718 & 15,2371 \\
& & & \\
50 & 0,895396 & 1,41322 & 15,4040
\end{tabular}

Table 18: Steady state values for parameters when varying number of discretizations

The differences in the results are not very large, suggesting that the dis- 
cretization is implemented in a correct way. Changing from 1 to 2 discretizations gives the largest change, as expected. The difference between 2 and 50 discretizations is $15.8 \%$ for hydrogen utilization, $1.4 \%$ for stack voltage and $2.2 \%$ for the total heat produced. For the fuel utilization the error is quite large, but for the voltage and heat good results are obtained already with 2 discretizations. Between 4 and 50 discretizations the difference is $8.2 \%$ for hydrogen utilization, $1.4 \%$ for stack voltage and $1.8 \%$ for the total heat produced. Still the utilization differs more than desired, but the voltage and heat results are very stable.

\subsubsection{Ohmic loss model with increasing current}

In this simulation the behaviour of a substack with the ohmic loss model is investigated. For this purpose a simulation is made with an external current described as a ramp between 1 and $150 \mathrm{~A}$. The simulation time is set to 48 hours so the system can be considered to be in steady-state at all times. The current density is then choosen as free variable to generate voltage versus current plots. The model parameters used in this simulation is given in Table 19 .

\begin{tabular}{lr} 
Parameter & Value \\
\hline$A S R_{0}[11]$ & $0.29 \Omega \mathrm{cm}^{2}$ \\
$T_{0}[11]$ & $1073 \mathrm{~K}$ \\
$E_{a}[11]$ & $0.65 \mathrm{eV}$ \\
$N$ & 4 \\
$A_{\text {cell }}[11]$ & $361 \mathrm{~cm}^{2}$ \\
$n_{\text {cell }}$ & 1 \\
$C_{\text {th,cell }}$ & $150.12 \mathrm{~J} / \mathrm{K}$ \\
Fuel molar fraction [2] & $26.3 \% \mathrm{H}_{2}, 17.1 \% \mathrm{CH}_{4}, 2.9 \% \mathrm{CO}$ \\
Air oxygen mass fraction & $4.4 \% \mathrm{CO}_{2}, 49.3 \% \mathrm{H}_{2} \mathrm{O}$ \\
Fuel inlet flow [2] & $23 \%$ \\
Air inlet flow [2] & $2.07 \cdot 10^{-5} \mathrm{~kg} / \mathrm{s}$ \\
Inlet temperatures & $9.422 \cdot 10^{-4} \mathrm{~kg} / \mathrm{s}$ \\
& $800^{\circ} \mathrm{C}$
\end{tabular}

Table 19: Model parameters used in simulation

The simulation results for stack voltage and power are shown as solid lines in Figure 26, together with simulation results from [11]. In this case the voltage was multiplied by 50 to represent a stack with 50 cells. The reference model is a OD SOFC model where the Nernst potential is evaluated using an average fuel composition between the stack inlet and outlet. For low currents the voltage and power error limits were calculated using the gas composition at the stack inlet for the lower error limit and using the gas composition at the stack outlet for the upper error limit. At high currents the gas composition at the stack outlet gives the lower error limits and the gas composition at the stack inlet gives the upper error limits.

For currents between 20 and $70 \mathrm{~A}$ the model has yielded stack voltage and power slightly higher than the upper error limit, but the results are still similar and for stack power the deviation is very small. The difference between the results is likely due to the different handling of heat in the two models. In [11] the 

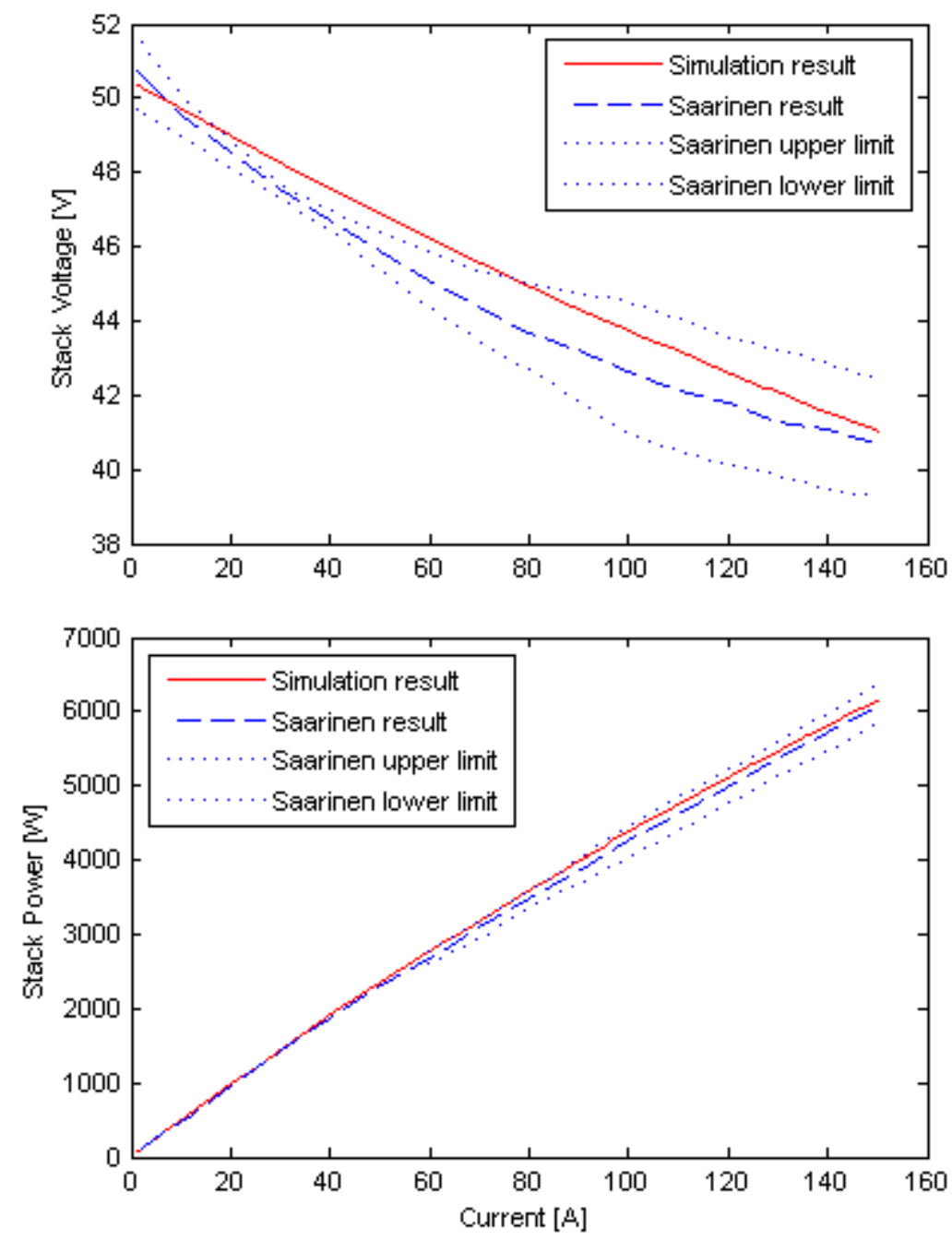

Figure 26: Simulation results for ohmic loss model in substack

stack was placed in a furnace which is kept at $760^{\circ} \mathrm{C}$ throughout the simulation, whereas the simulated stack was cooled only by heat transfer between the stack gases and the inner surfaces of the fuel and air channels. Figure 27 shows the temperature in all four discretizations of the stack, together with a reference line at $760^{\circ} \mathrm{C}$.

For the case of $20 \mathrm{~A}$, some discretizations have a higher temperature than $760^{\circ} \mathrm{C}$, giving them a lower area specific resistance and thereby contributing to a higher stack voltage. 


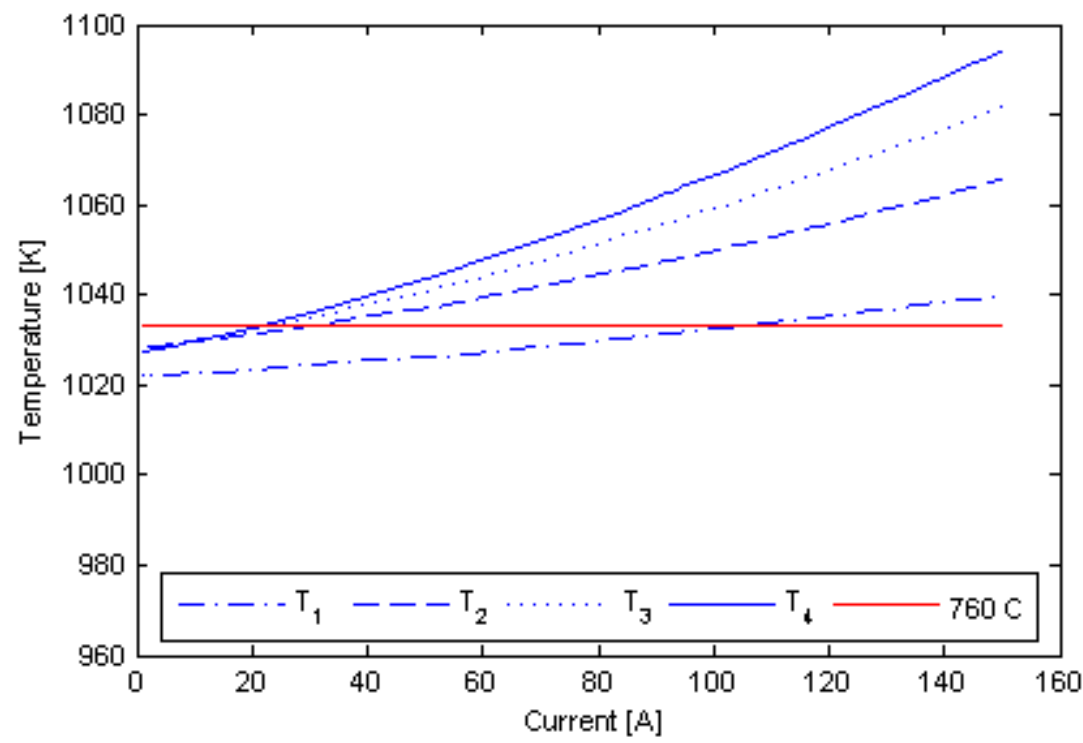

Figure 27: Temperature in stack discretizations 


\subsubsection{Electrochemical model startup dynamics}

In this simulation the transient behaviour of the substack and the complete electrochemical model with a constant external current is investigated. The initial temperature of the stack is set $800^{\circ} \mathrm{C}$ and the fuel and air inlet temperature is set to $900^{\circ} \mathrm{C}$ and therefore there will be a transient in the temperature, and thereby also in the stack voltage and losses. In the simulation the parameters in Table 14 and the geometry in Table 20 are used. Note that in Table 20 the anode and cathode thicknesses refers to the total thicknesses $t_{a}+t_{c c s}$ and $t_{c}+t_{c c c}$ using the notation in Figure 6. Thus the current collector thicknesses refers to the part of the electrodes where in-plane currents are present. This distinction is only used in the calculation of the ohmic resistance. The fuel composition represents that of reformed natural gas. The geometry describes typical values for an anode supported cell described in literature. The number of cells in the stack is set to 50 to obtain slower temperature dynamics and the fuel and air flows are increased to $1.035 \cdot 10^{-3} \mathrm{~kg} / \mathrm{s}$ and $4.711 \cdot 10^{-2} \mathrm{~kg} / \mathrm{s}$, respectively. The spliceValue parameter is set to $0.025 \mathrm{~V}$. All parameters and operating conditions given in [2] for the $5 \mathrm{~kW}$ SOFC stack manufactured by Forschungszentrum Jülich (FZJ), Germany, are used. Some model parameters are not given there and have been taken from other sources, as stated in the tables.

\begin{tabular}{lr} 
Parameter & Value \\
\hline Anode thickness & $1,01 \mathrm{~mm}$ \\
Anode current collector thickness & $1 \mathrm{~mm}$ \\
Cathode thickness & $70 \mu \mathrm{m}$ \\
Cathode current collector thickness & $60 \mu \mathrm{m}$ \\
Electrolyte thickness & $10 \mu \mathrm{m}$ \\
Cell pitch length & $5 \mathrm{~mm}$ \\
Active cell area & $361 \mathrm{~cm}^{2}$
\end{tabular}

Table 20: Cell geometry used in simulation

The system is simulated for 45 minutes with an external current of $150 \mathrm{~A}$, corresponding to a current density of $4155 \mathrm{~A} / \mathrm{m}^{2}$. In Figure 28 the transient behaviour of the stack voltage, temperature, utilization and power generation is presented.

The transient temperature is shown in Figure 28(b) and it takes approximately 30 minutes for it to reach steady-state, which is also the case for simulations in [2]. The temperature is highest in the first discretization, due to a high heat production in this discretization. In chapter 5.3.6 it was shown that the dominant losses decrease at higher temperatures and as a consequense the stack voltage increases, as shown in Figure 28(a). The stack reaches a maximum voltage of $48.5 \mathrm{~V}$ which corresponds to a cell voltage of $0.97 \mathrm{~V}$.

Utilization of methane and hydrogen is shown in Figure 28(c) and neither of them reaches 1 at any time. This means that more fuel is supplied than what would be requiered to avoid the severe voltage drop that occurs when the fuel is depleted. This case is investigated in chapter 5.4.5. After the initial transient the hydrogen utilization increases with increased temperature, and the 

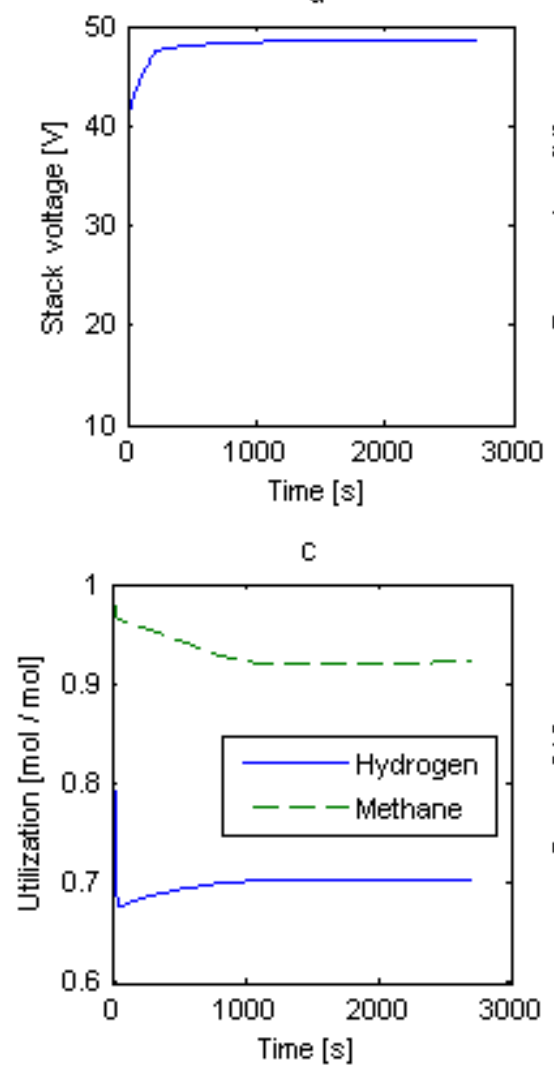

b

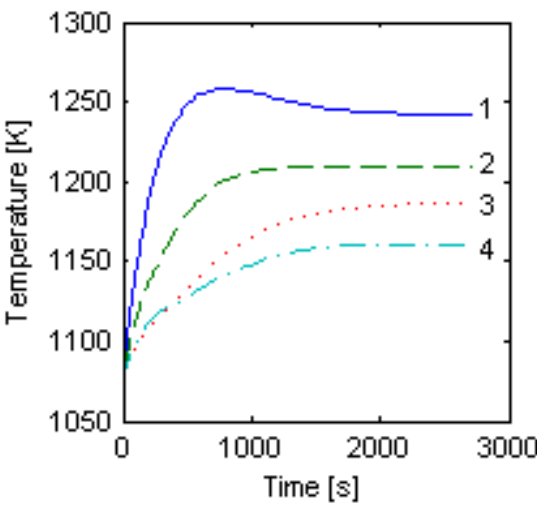

d

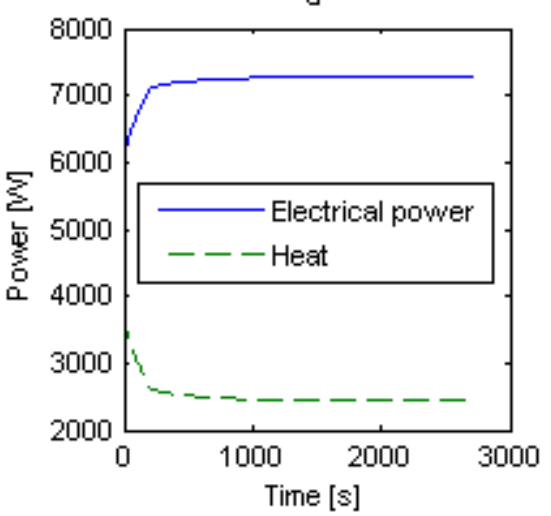

Figure 28: Transient behaviour of: (a) Stack voltage, (b) Temperature in discretizations numbered from inlet to outlet, (c) Utilization of fuel hydrogen and methane, (d) Total power and heat generated

cell power production also increases, as expected. In this simulation the incorrect utilization measure given in equation 33 was used, and the values obtained therefore may be a bit to large.

In Figure 28(d) the power generated in the external load and the total amount of heat produced inside the stack are shown. In the initial transient much heat is generated which increases the temperature of the stack. When a higher temperature is reached the losses are lower and thereby less heat is produced and more power is generated in the external load.

Figure 29 shows the current density and ohmic loss in each discretization, which have a direct relation via Ohm's law and are therefore expected to show similar characteristics. The current density is highest in the first discretization, indicating that the highest reaction rate of hydrogen is found here. The current density then decreases towards the stack oulet.

The activation losses for the stack are shown in Figure 30. The activation 
a

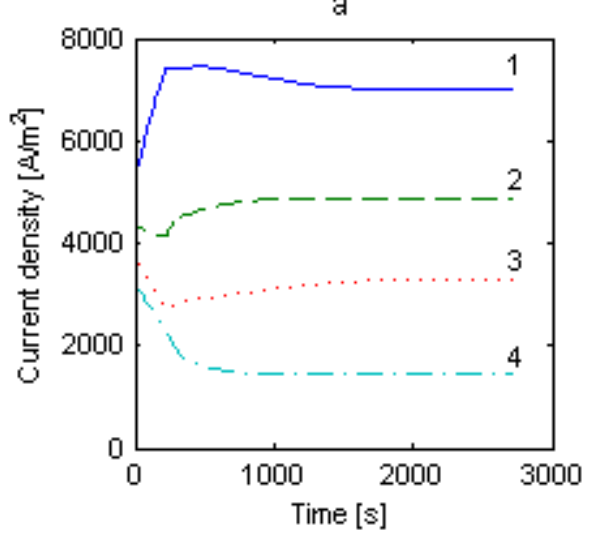

b

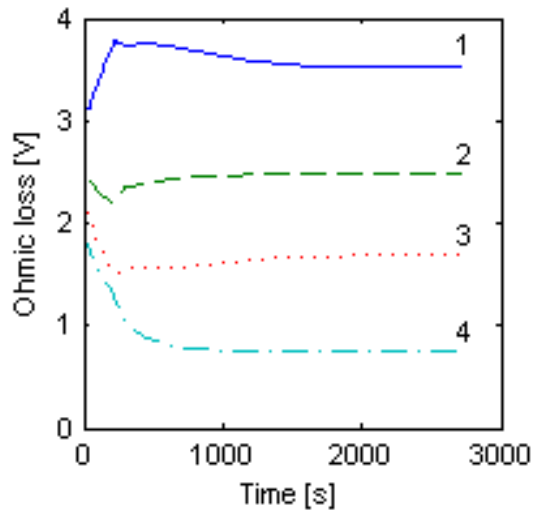

Figure 29: Transient behaviour of: (a) Current density in each disctretization, (b) Ohmic loss in each discretization, numbered from inlet to outlet.

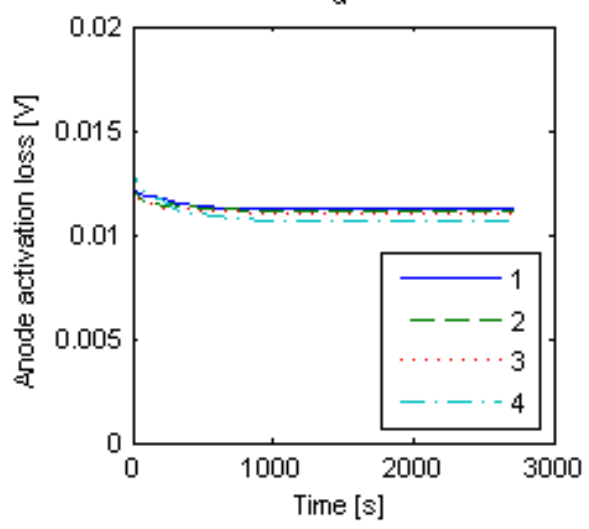

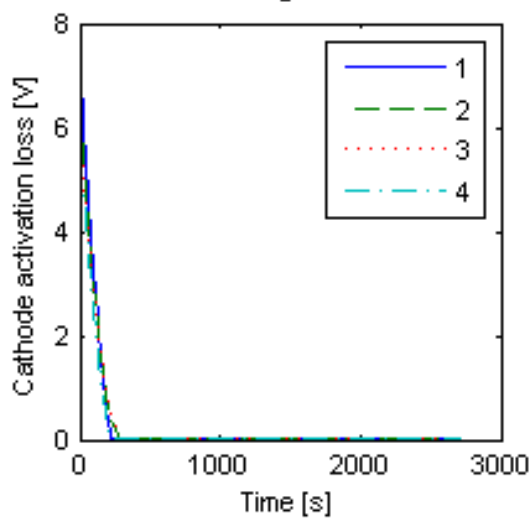

Figure 30: Transient behaviour of: (a) Anode activation loss in each discretization, (b) Cathode activation loss in each discretization

loss at the anode side is small at all times, as can be seen in Figure 30(a), and there is also very little difference between the discretizations. This can be expected when the current density in each discretization is lower than the exchange current density [1].

At the cathode side there is initially a considerable activation loss, as seen in Figure 30(b). The loss decreases quickly, however, and since there is no drastical decrease in the current density the exchange current density must be increasing. According to equations (21) and (22) the exchange current density will increase when the temperature is increased.

In Figure 31 the concentration loss and the diffusion coefficients for hydrogen and steam are shown. A low concentration loss can be expected as the fuel utilization never approaches 1 . The diffusion coefficients does not show much 
variation and it can be seen that hydrogen diffuses quicker than steam in this gas mixture.
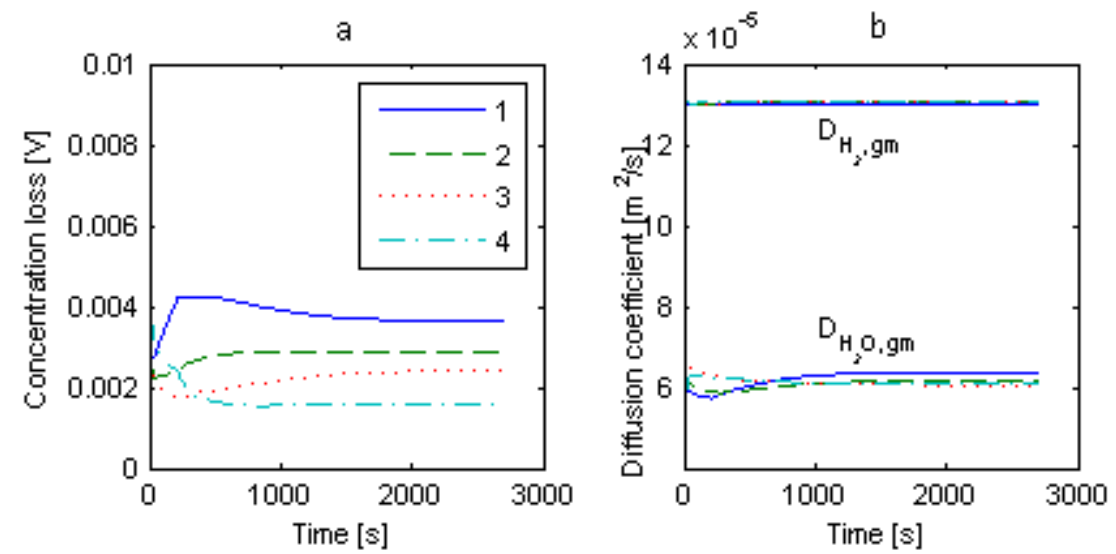

Figure 31: Transient behaviour of: (a) Concentration loss in each discretization, (b) Diffusion coefficients for hydrogen and steam in each discretization 


\subsubsection{Electrochemical model with increasing current}

In this simulation the behaviour of the substack is investigated with an external current described by a ramp. The cell geometry given in Table 20 are used and the number of cells in the stack is set to 1 to obtain voltages per cell. The fuel and air compositions in Table 19 is used at the given temperature, but the flow rates are decreased to $4.1 \cdot 10^{-5} \mathrm{~kg} / \mathrm{s}$ for the fuel and $20 \cdot 10^{-4} \mathrm{~kg} / \mathrm{s}$ for the air as there is only one cell in the stack. The simulation runs for 48 hours so that the system can be considered to be in steady state for all times. During this time the external current is increased linearly from $10 \mathrm{~A}$ to $520 \mathrm{~A}$, which corresponds to a cell current density of $277 \mathrm{~A} / \mathrm{m}^{2}$ to $14404 \mathrm{~A} / \mathrm{m}^{2}$.

The results from the simulation are shown in Figures $32-37$.

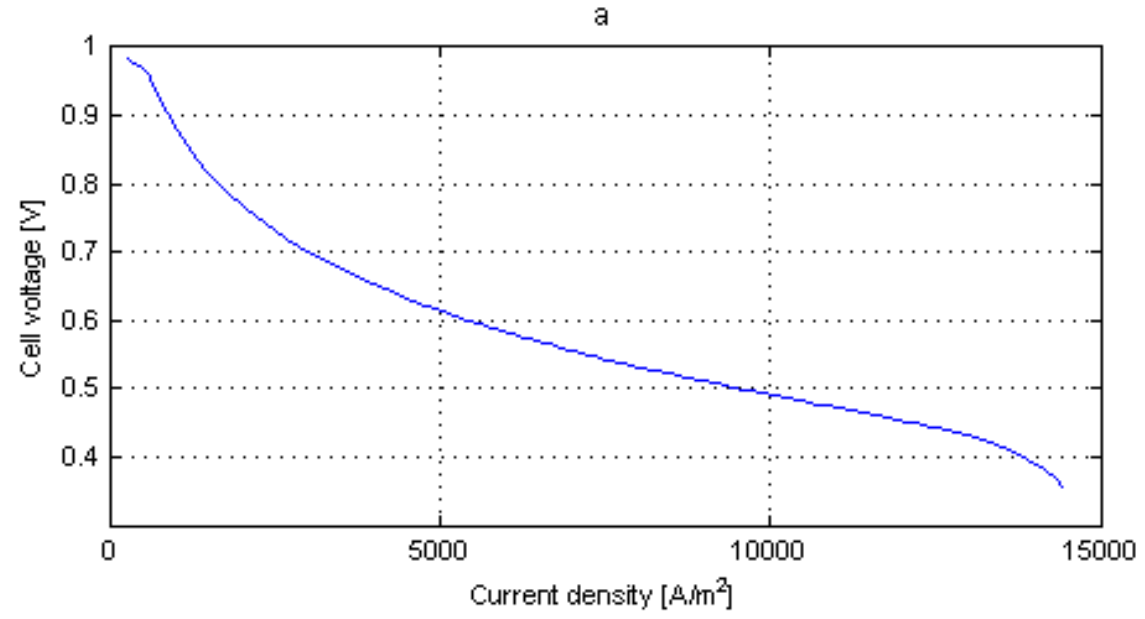

b

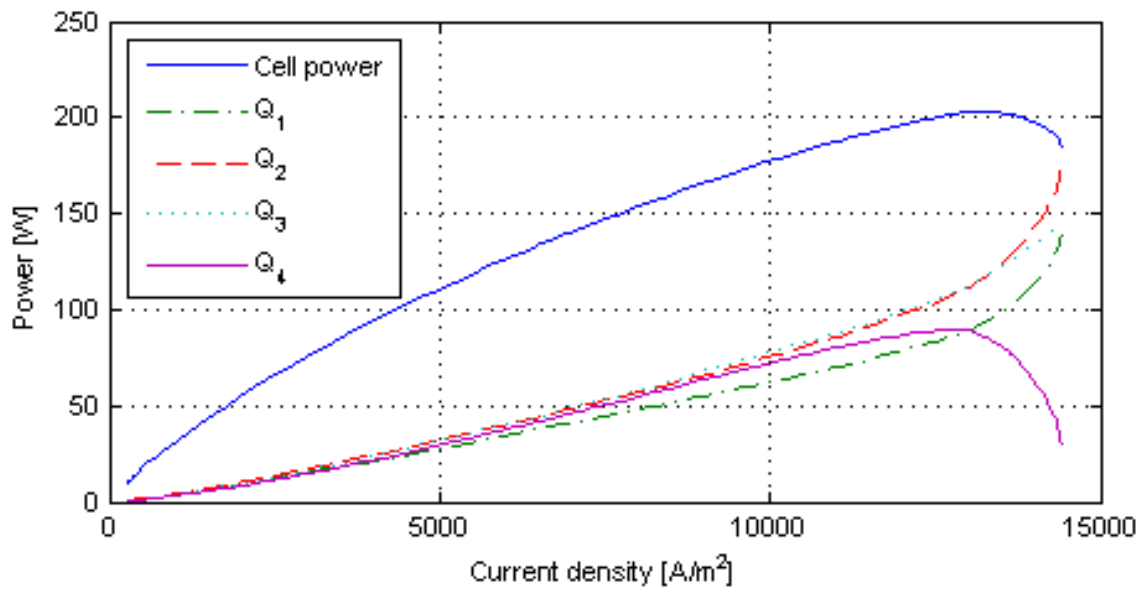

Figure 32: (a) Cell voltage versus overall cell current density, (b) Total power generation and heat generation per discretization versus cell current density 
Shown in Figure 32(a) is the cell voltage versus the cell current density which has the expected characteristics with a fast voltage drop for low current densities, a close to linear behaviour for medium current densities and then a faster drop at high current densities.

In Figure 32(b) the total cell power is shown together with the heat generated in each discretization. The cell power increases with increased current until the voltage drop leaves its linear region at the high current densities. The maximum power generation is in this case obtained at a current density of $13000 \mathrm{~A} / \mathrm{m}^{2}$. The heat generation also grows with increased current density and roughly the same amount of heat is produced in each discretization, except for very high currents when the heat production in the 4 th discretization decreases rapidly. This behaviour derives from the quick decrease in current through the discretization closest to the stack outlet for very high currents that is shown in figure 35(a), as the heat produced is given by the current through the discretization and its electrical resistance.

a

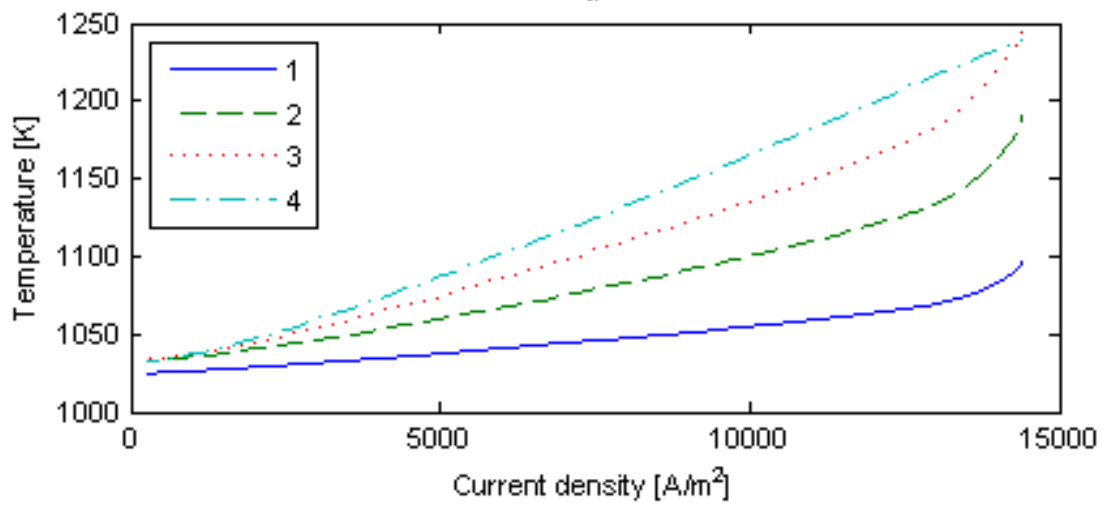

b

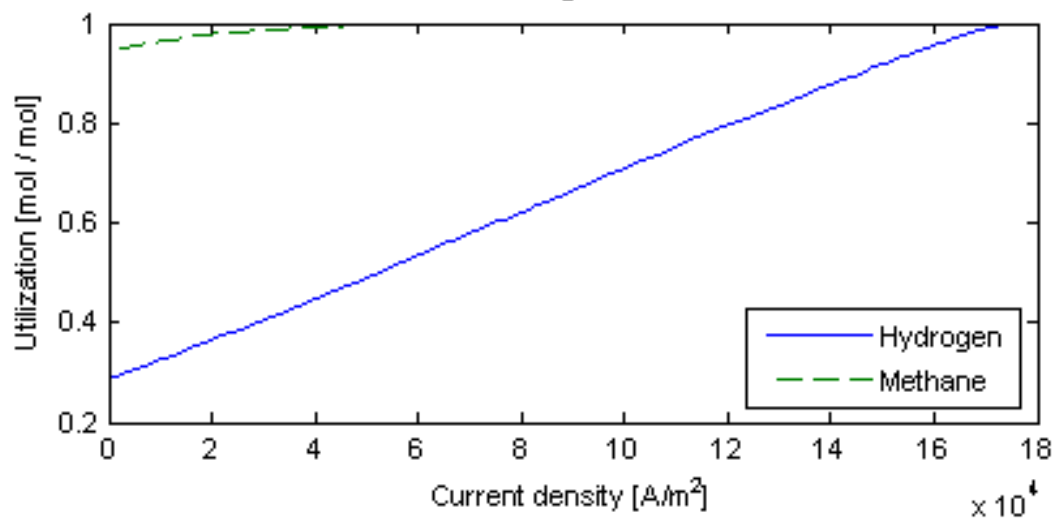

Figure 33: (a) Temperature for each discretization versus cell current density, (b) Fuel hydrogen and methane utilization versus cell current density

The temperature in each discretization is shown in Figure 33(a). In general 
the stack is coolest at the fuel inlet and gets hotter towards the outlet. The main reason for this is that fuel entering the stack is cooler than the stack itself, and that the major part of the methane reforming takes place in the first discretizations. The reforming reaction (5) is endothermic, and thereby lowers the fuel temperature.

In Figure 33(b) the fuel utilization is shown. For current densities lower than $5000 \mathrm{~A} / \mathrm{m}^{2}$ the utilization of methane is not close to 1 , indicating that not all methane has been internally reformed and thereby the exhaust gas from the stack outlet still contains unused methane. Operating the fuel cell in this way is very inefficient, unless the exhaust gas is further processed to make use of the remaining methane and hydrogen. The hydrogen utilization increases almost lineraly with increased current and when it approaches 1 the cell voltage drops rapidly. When operating the fuel cell with hydrogen utilization close to 1 the part of the cell closest to the outlet contributes only mariginally to the total cell power generation due to the low hydrogen content in the fuel. In this simulation the incorrect utilization measure given in equation 33 was used, and the values obtained therefore may be a bit to large.

In Figure 34(a) the methane molar fraction of the fuel is shown for each discretization. The graph shows the gradual decrease in methane content along the fuel flow path due to the methane reforming. For current densities above $5000 \mathrm{~A} / \mathrm{m}^{2}$ there is not much methane present in the fuel and for very high currents even the methane content in the first discretization is very low.

In Figure 34(b) the hydrogen content in each discretization is shown. For very low current densities the hydrogen content is actually higher in the last discretization, which indicates that more hydrogen is produced from methane reformation than consumed by the electrochemical reaction. At higher current densities the reaction rate is greater than the refomation rate, and the hydrogen content decreases along the fuel flow path.

The fuel steam content is presented in Figure 34(c) and it is clear that when the hydrogen content is decreased in a discretization, the steam content is increased. This is due to the fact that for every part of hydrogen combusted one part of steam is produced.

In Figure 34(d) the air oxygen content is shown and it has the same characteristics as the hydrogen graph. This is also due to the fact that for every part of hydrogen combusted, one part of oxygen must be supplied to the reaction site from the air at the cathode side, via flow of oxygen ions through the electrolyte.

Figure 35(a) shows the current density in each discretization versus the total cell current density. For low and medium current densities the variation in current density over the cell is rather small, but at high current densities the current through the last discretization decreases rapidly. The reason for this decrease is the absence of fresh fuel in the bulk flow, which prevents combustion of hydrogen (equation 3).

The ohmic losses in each discretization, presented in Figure 35(b), has a 
(a) Fuel methane content

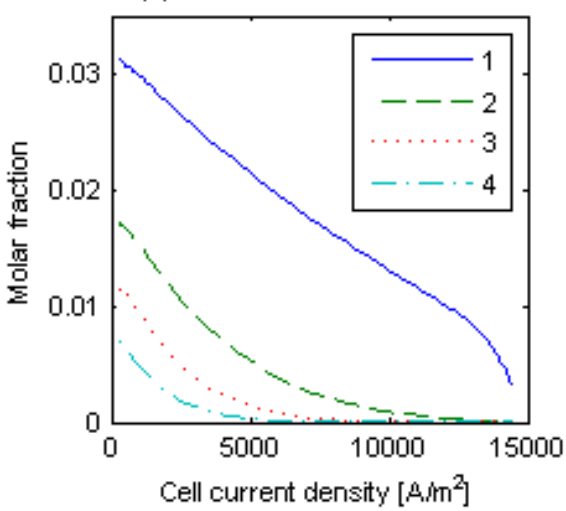

(c) Fuel steam content

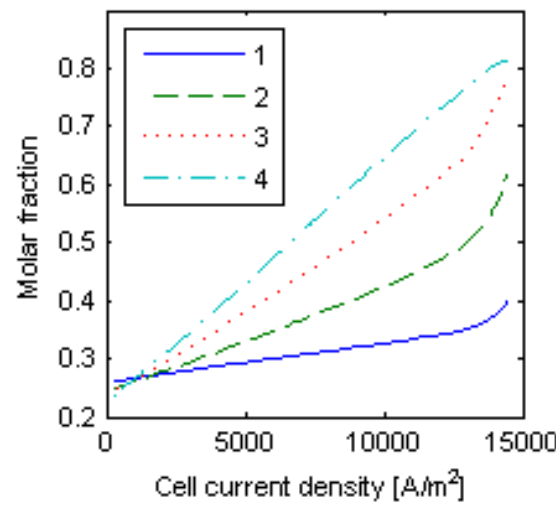

(b) Fuel hydrogen content

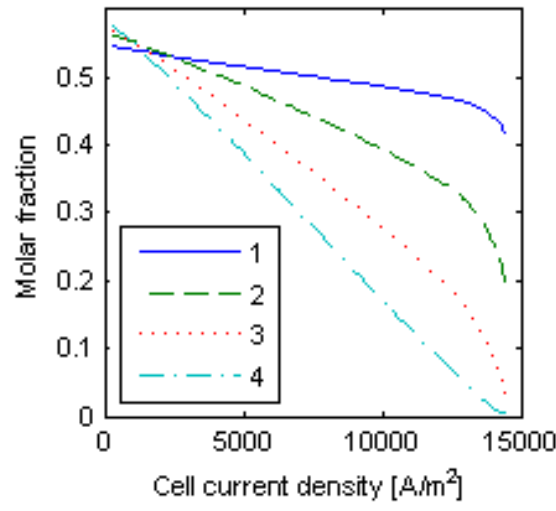

(d) Air oxygen content

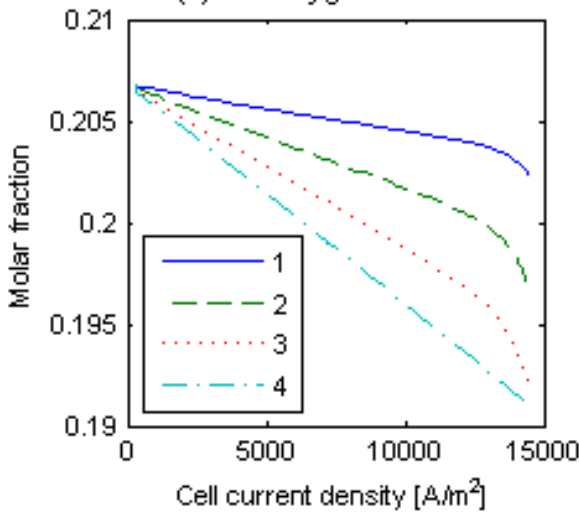

Figure 34: (a) Fuel methane molar fraction versus cell current density, (b) Fuel hydrogen molar fraction versus cell current density, (c) Fuel steam molar fraction versus cell current density, (d) Air oxygen molar fraction versus cell current density

behaviour very similar to that of the current density. This is because of the ohmic losses direct relation to the current via the Ohm's law.

The remaining losses are presented in Figure 36. In Figure 36(a) the anode activation loss in each discretization is shown. It is very low for most cases in this simulation, except for the last discretization at high current densities. The low values indicate that the cell current density is lower than the anode exchange current density, which depends on the fuel hydrogen and steam content according to equation (22).

The cathode activation loss presented in Figure 36(b) has larger values than the anode activation loss. In this case the cell current density is larger than the cathode exchange current density and the loss is given by the Tafel equation (19). As shown in Figure 35(a) the current density in the last discretization decreases at high cell current densities and as a consequence the cathode activation loss decreases in this discretization, as the current density here approaches 


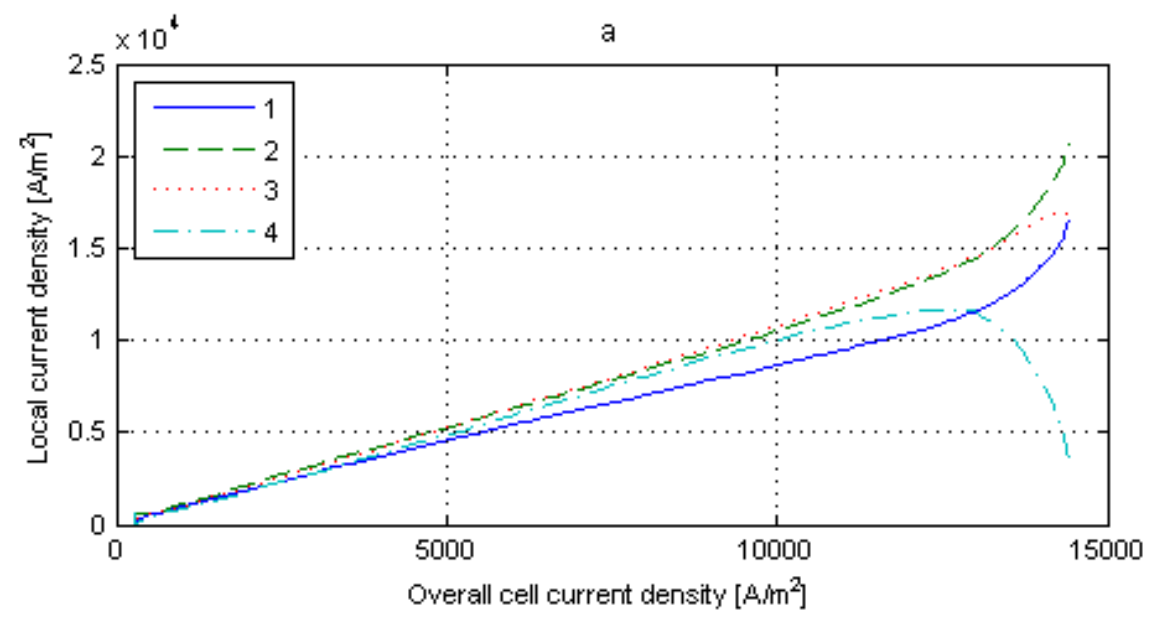

b

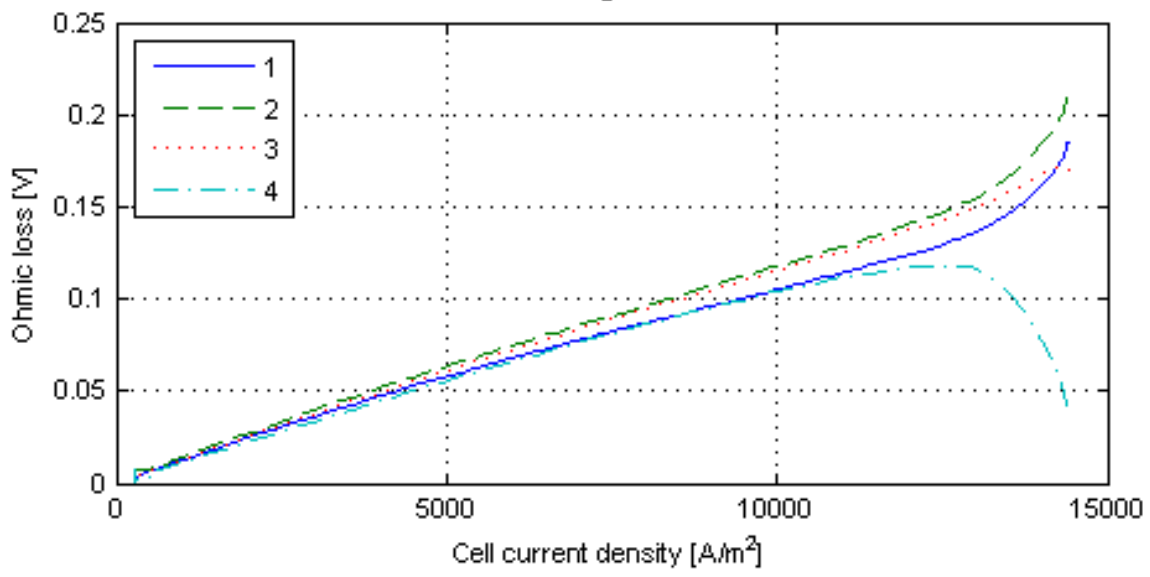

Figure 35: (a) Current density per discretization versus cell current density, (b) Ohmic loss per discretization versus cell current density

the cathode exchange current density.

The concentration loss in each discretization is shown in Figure 36(c). The concentration loss is very low for most cases, but increases rapidly in the last two discretizations when the hydrogen utilization approaches 1 at high current densities.

In Figure 37 the diffusion coefficients for the hydrogen and the steam in the gas mixture are shown. The diffusion coefficeint is a measure of how easily species diffues through a medium and thereby affects the concentration loss. A higher diffusion coefficient gives a lower concentration loss and vice versa.

There is a larger variation of the diffusion coefficient and this is because of the large variation of the fuel composition during a simulation with large changes 
in the current density. For example when the fuel steam content increases in the two last discretizations for high current densities the diffusion coefficent for steam in the gas mixture decreases, which gives a large concentration loss in these discretizations. 

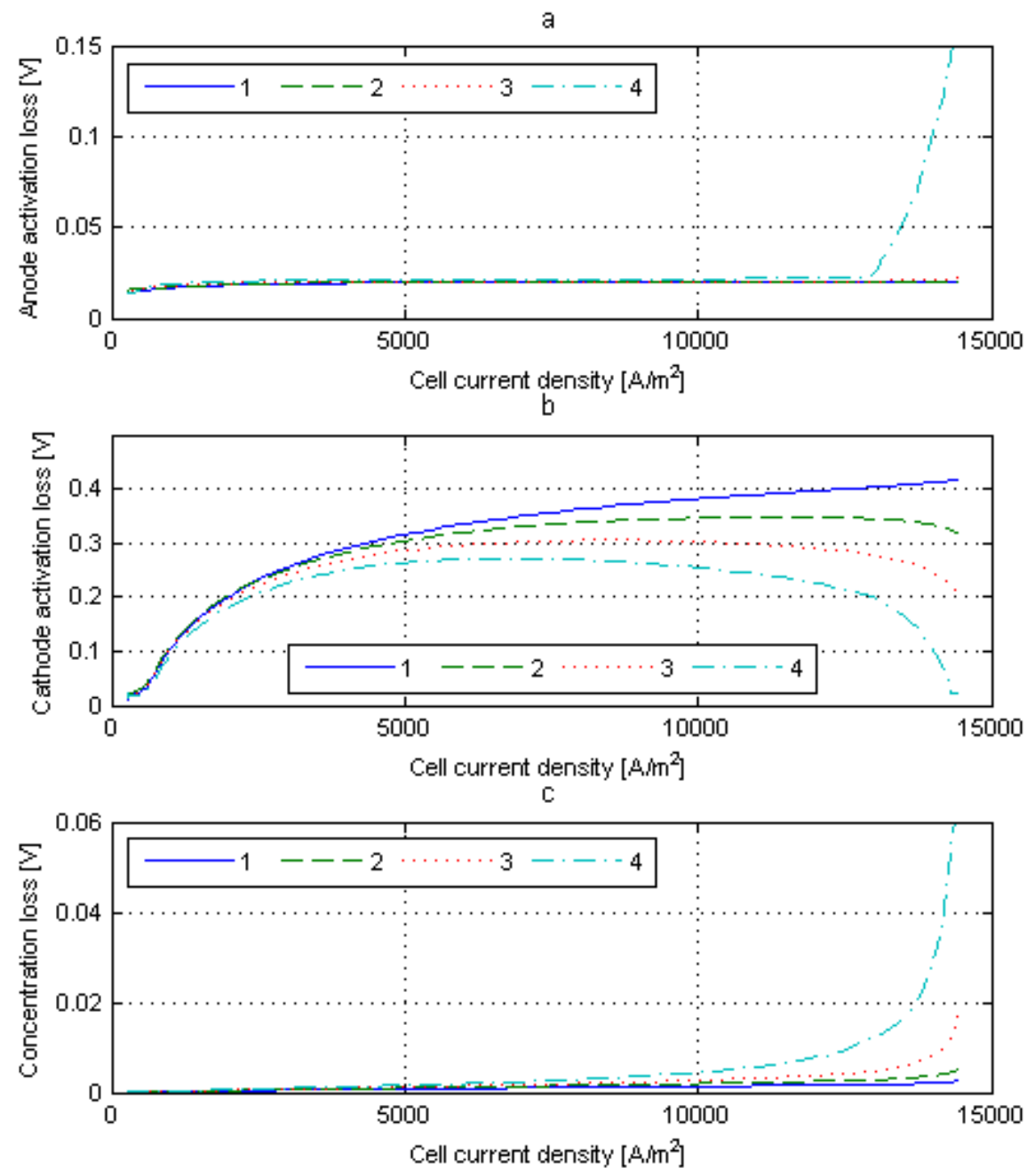

Figure 36: (a) Anode activation loss per discretization versus cell current density, (b) Cathode activation loss per discretization versus cell current density, (c) Concentration loss per discretization versus cell current density 

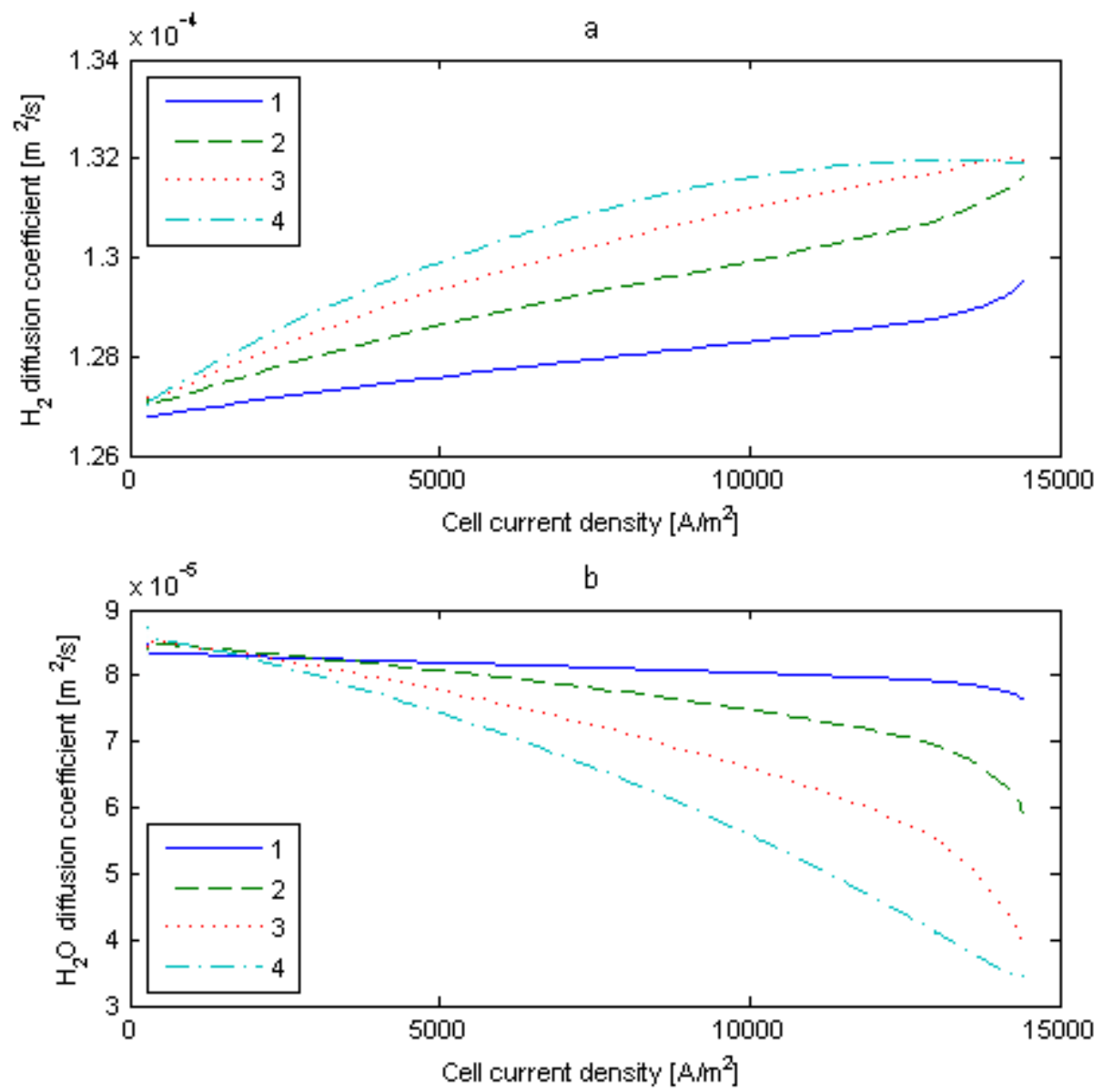

Figure 37: (a) Diffusion coefficient of hydrogen in fuel mixture per discretization versus cell current density, (b) Diffusion coefficient of steam in fuel mixture per discretization versus cell current density 


\subsection{Stack model validation}

Figure 38 shows the setup used for validation of the stack model.

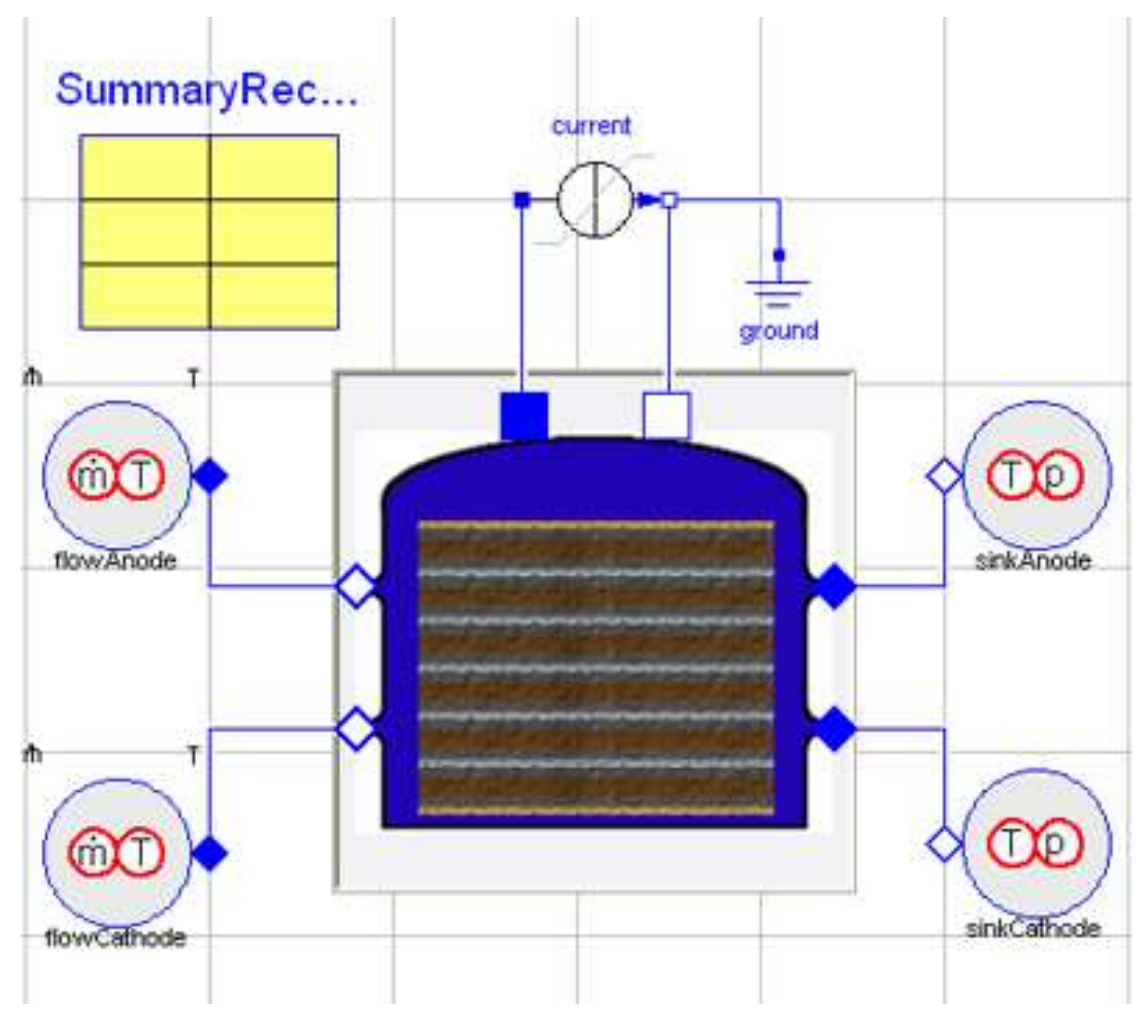

Figure 38: Experimental setup for validation of the stack model

A current is generated between the electrical pins of the stack. Fuel and air supply sources are connected to the stack inlets and sinks are connected to the outlets. The stack contains 50 cells divided between three substacks connected in series. The top and bottom substacks contains 10 cells each and the middle substack contains 30 cells. The nominal pressure drop in the manifold volumes is set to $100 \mathrm{~Pa}$, which means that the pressure in the first substack will be approximately $100 \mathrm{~Pa}$ higher than the pressure in the third substack. The cells are modeled by the complete electrochemical model described in chapter 2.3 and the geometry data shown in Table 20 is used. A constant external current of $150 \mathrm{~A}$ is used in the simulations. This corresponds to a current density of $4155 \mathrm{~A} / \mathrm{m}^{2}$. The initial temperature of the stack is set to $800^{\circ} \mathrm{C}$, which is also the temperature of the gas fed to the stack. The inlet flow rates of fuel and air are set to $1.035 \cdot 10^{-3} \mathrm{~kg} / \mathrm{s}$ and $4.711 \cdot 10^{-2} \mathrm{~kg} / \mathrm{s}$, respectively, and the fuel composition was set to that of the ATR outlet from simulations, given in Table 23 . All other operating conditions are the same as used in the dynamic simulation of the electrochemical cell model in a substack, presented in section 5.4.4. The system was simulated for 45 minutes with these parameters. Results from the simulations are shown in Figure 39. 
Figure 39(a) shows the voltage of the complete stack, and that of the different substacks. Note that the voltages of substacks 1 and 3 overlaps. The total stack voltage reaches $32.306 \mathrm{~V}$ in steady-state, which corresponds to a cell voltage of $0.6461 \mathrm{~V}$. The middle substack, (substack 2), has a steady-state cell voltage of $0.6832 \mathrm{~V}$, which is much higher than that of the other substacks. The cell voltage is $0.5921 \mathrm{~V}$ and $0.5888 \mathrm{~V}$ in substacks 1 and 3, respectively. The higher cell voltage in the middle substack can be explained by the temperature distribution between the substacks, presented in Figure 39(b). The steady-state temperature of the middle substack is $1094^{\circ} \mathrm{C}$. This gives smaller ohmic losses than in the top and bottom substacks, where the temperatures are $1010^{\circ} \mathrm{C}$ and $1012^{\circ} \mathrm{C}$, respectively. The small differences in temperature and cell voltage between the top and bottom substacks is a consequense of pressure drop in the manifold volumes. The lower pressure in the bottom substack leads to a lower inlet flow rate to that substack, which in turn leads to a higher concentration loss than in the top substack.

Utilization of hydrogen gas in the substacks are presented in Figure 39(c). The utilization is calculated according to equation (34). The steady-state hydrogen utilization is 0.69 in substack $1,0.65$ in substack 2 and 0.75 in substack 3 . The high utilization in substack 3 can be explained by the lower flow rate through the substack, since low flow rate means longer residence time in the substack channel. Longer residence time increases the chances for reactions to occur.

Power generation and heat production in the stack and in the substacks are shown in Figure 39(d). Note that for both power and heat production, the results for substacks 1 and 3 overlaps. The total power generated by the stack is $4846 \mathrm{~W}$ in steady-state. This is of course a good result as the reference stack in [2] is a $5 \mathrm{~kW}$ SOFC stack.

The overall impression of the results is that the stack shows reasonable behaviour and performance when compared to the substack validation in section 5.4.4 and to the behaviour of SOFC stacks in literature [2]. 


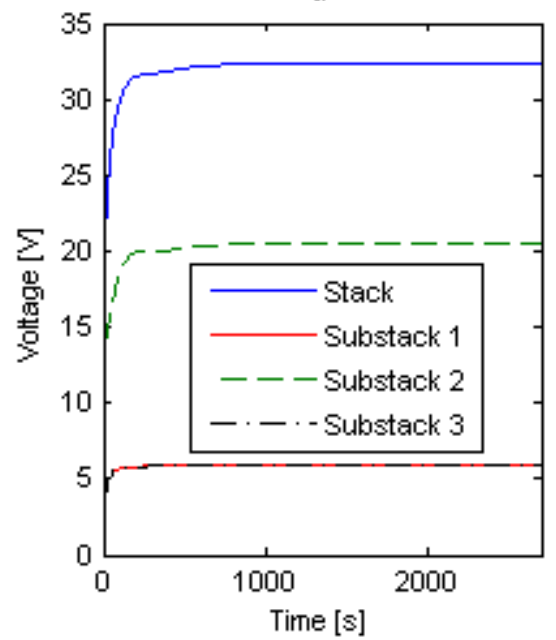

b

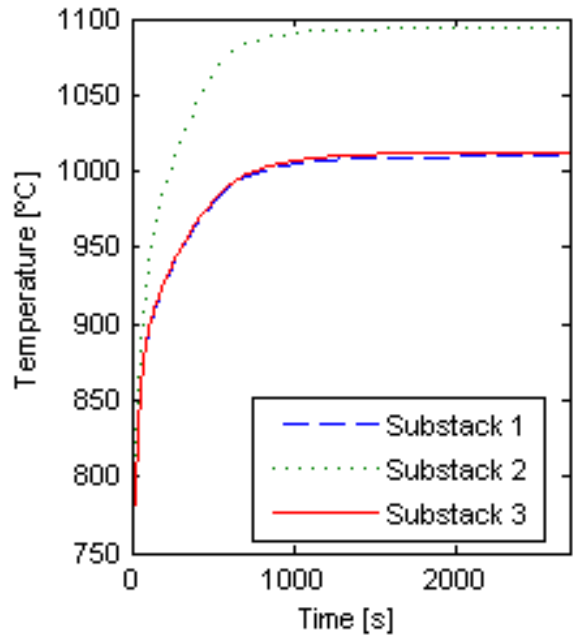

$\mathrm{C}$
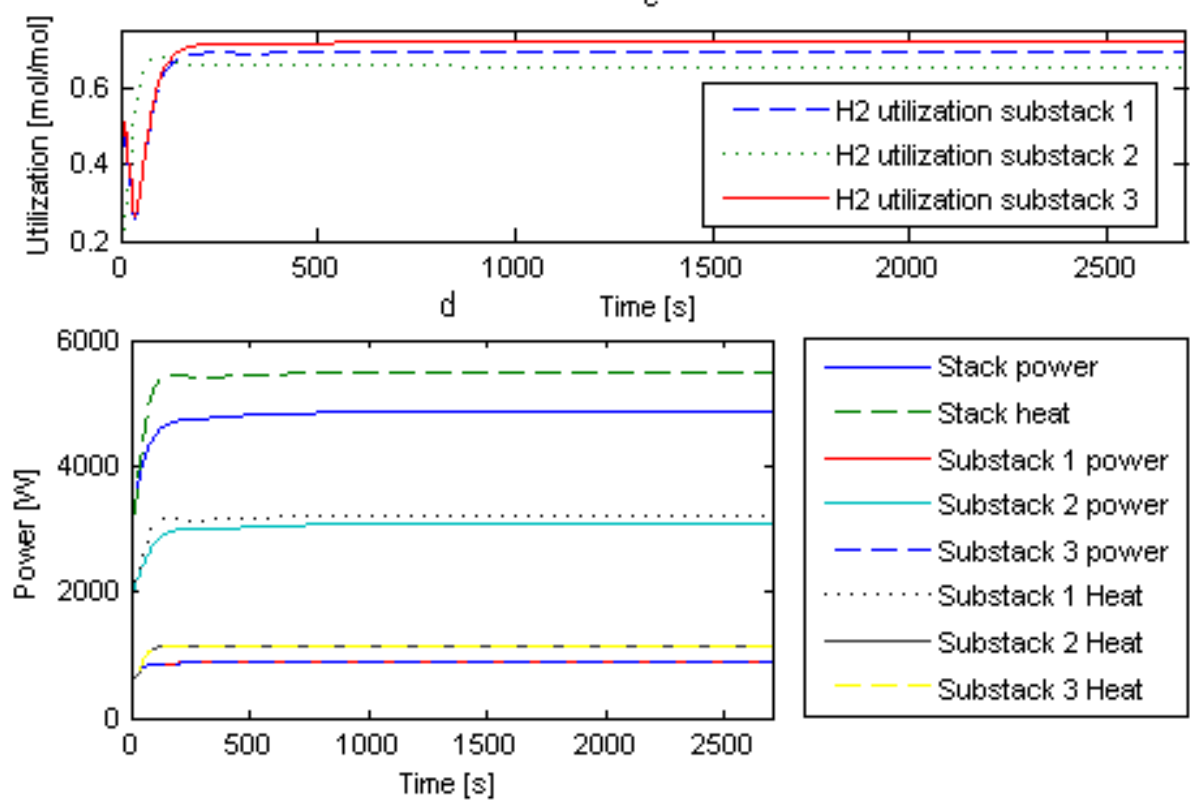

Figure 39: Results from the dynamic stack simulation: (a) Stack and substack voltages, (b) Temperature in substacks, (c) Utilization of hydrogen in substacks, (d) Heat and power in stack and substacks. 


\subsection{Verification of system components}

\subsubsection{Autothermal reformer}

The experimental setup used for validation of the autothermal reformer is shown in Figure 40. The temperature in the reformer was set to a constant value of $500^{\circ} \mathrm{C}$ and the pressure in the sink was changed so that a pressure close to 1.5 bar was achieved in the reformer. Other test parameters used in the simulation are presented in Table 21.

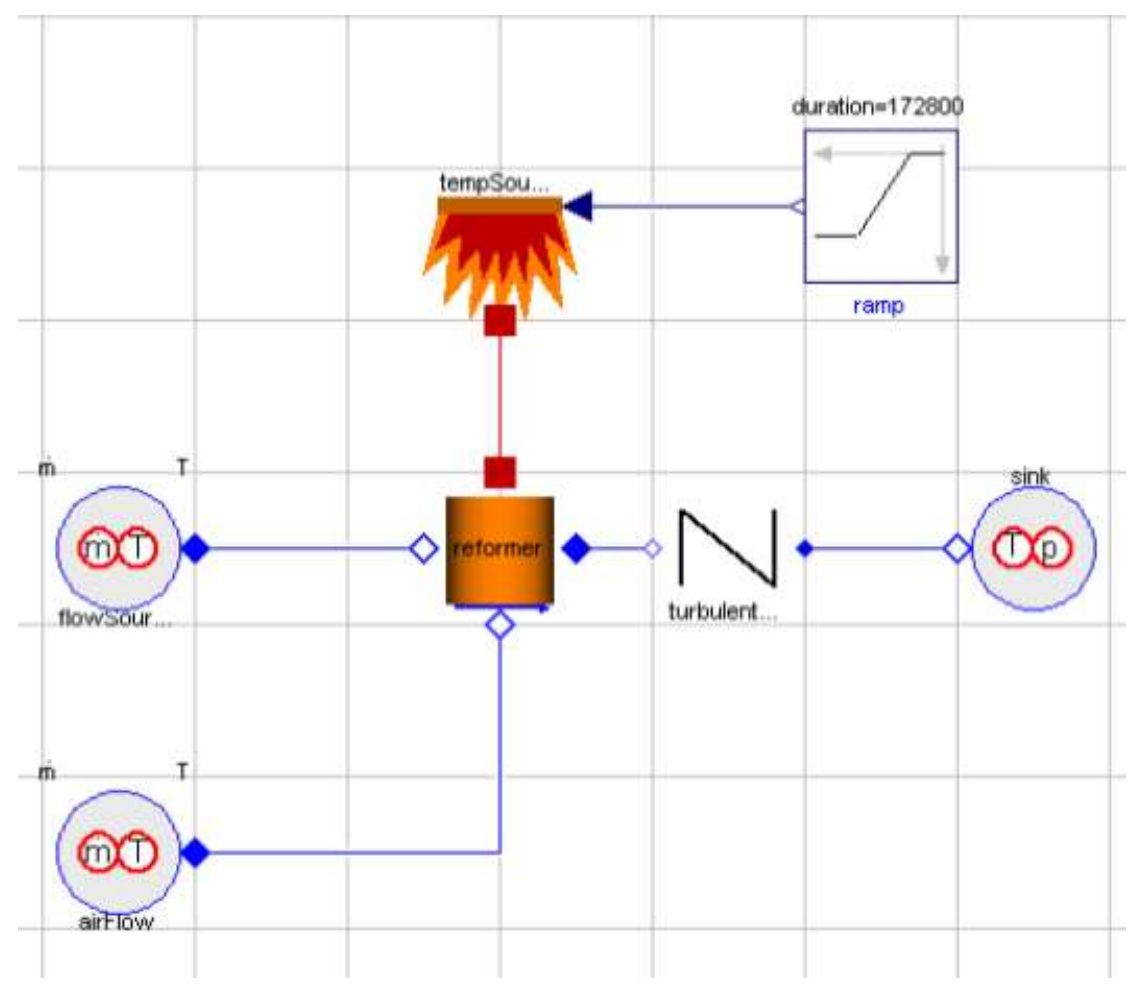

Figure 40: Experimental setup for validation of the autothermal reformer

Two different simulations were done with these parameters in order to show the influence of different inlet compositions on the system efficiency. The simulation results were compared with data from literature [20]. In the first simulation, oxygen gas carbon ratio $(\mathrm{O} / \mathrm{C})$ was kept constant at 0.45 while the steam carbon ratio $(\mathrm{S} / \mathrm{C})$ was ramped in the range $0.5-12$. The simulation results are shown in Figure 41.

The figure shows the behavior of the $\mathrm{CH}_{4}$ conversion, the reforming efficiency and the dry mole fractions of $\mathrm{H}_{2}$ at different $\mathrm{S} / \mathrm{C}$ ratios. It is obvious that small changes in the composition ratios can have a major impact on the reformer efficiency. The resulting values are growing with higher ratios but becomes slow as the ratio becomes larger. The behaviour of the ATR unit is similar to the behaviour of the reformer used in [20] but some differences exists at high $\mathrm{S} / \mathrm{C}$ ratios. One major difference is that the thermal reforming efficiency 
Reformer parameters

\begin{tabular}{lr}
\hline Volume & $0.0024 \mathrm{~m}^{3}$ \\
Initial temperature & $500{ }^{\circ} \mathrm{C}$ \\
Initial pressure & $1.6 \mathrm{bar}$ \\
Nominal mass flow rate & $9 \cdot 10^{-4} \mathrm{~kg} / \mathrm{s}$ \\
$L_{H} V_{H_{2}}$ & $240 \mathrm{MJ} / \mathrm{kmol}$ \\
$L H V_{C H_{4}}$ & $800 \mathrm{MJ} / \mathrm{kmol}$ \\
Sources & $500{ }^{\circ} \mathrm{C}$ \\
\hline Temperature & \\
Total mass flow rate (fuel + air) & $9 \cdot 10^{-4} \mathrm{~kg} / \mathrm{s}$ \\
& \\
Inlet fuel composition ratios & 0 \\
\hline $\mathrm{H}_{2} / \mathrm{NG}$ & 0.03 \\
CO $/$ C & 0 \\
CO/C & \\
turbulentLoss & \\
\hline Nominal density & $1 \mathrm{~kg} / \mathrm{m}^{3}$ \\
Nominal pressure drop & $0.01 \mathrm{bar}$ \\
Sink & \\
\hline Pressure in sink & $1.5 \mathrm{bar}$
\end{tabular}

Table 21: Parameters used when validating the autothermal reformer

doesn't seem to reach its stationary value in Figure 41. This may be explained by differences between the implementations. It is not obvious how the reformer in [20] was implemented. Assumptions made in that implementation may have given rise to the differences between the models.

Figure 42 shows the results from the second simulation. Here, the $\mathrm{O} / \mathrm{C}$ ratio was ramped in the range 0.01-1.5 while the $\mathrm{S} / \mathrm{C}$ ratio was kept constant at 6 . It is shown that a oxygen gas carbon ratio close to 1 gives a lower reformer efficiency than if the ratio was 0.3 . The reason is that the higher oxygen content leads to more total combustion, reaction (39), in the pre-reformer. This will decrease the content of methane which leads to lower efficiency. The results are within a reasonable range and complies with the values given in [20] for larger $\mathrm{O} / \mathrm{C}$ ratios. There are some differences between the models at lower $\mathrm{O} / \mathrm{C}$ ratios. As before, this is hard to explain without full insight in the implementation of the reformer in [20]. Even though there are some differences, it can be concluded that the reformer shows reasonable behavior, compared to the results presented in $[20]$.

Dynamic simulations of the autothermal reforming unit was also carried out in order to show its dynamic behavior. The test model in Figure 43 was used for this purpose.

The inlet fuel composition ratios and mass flow rate of air and fuel were set 


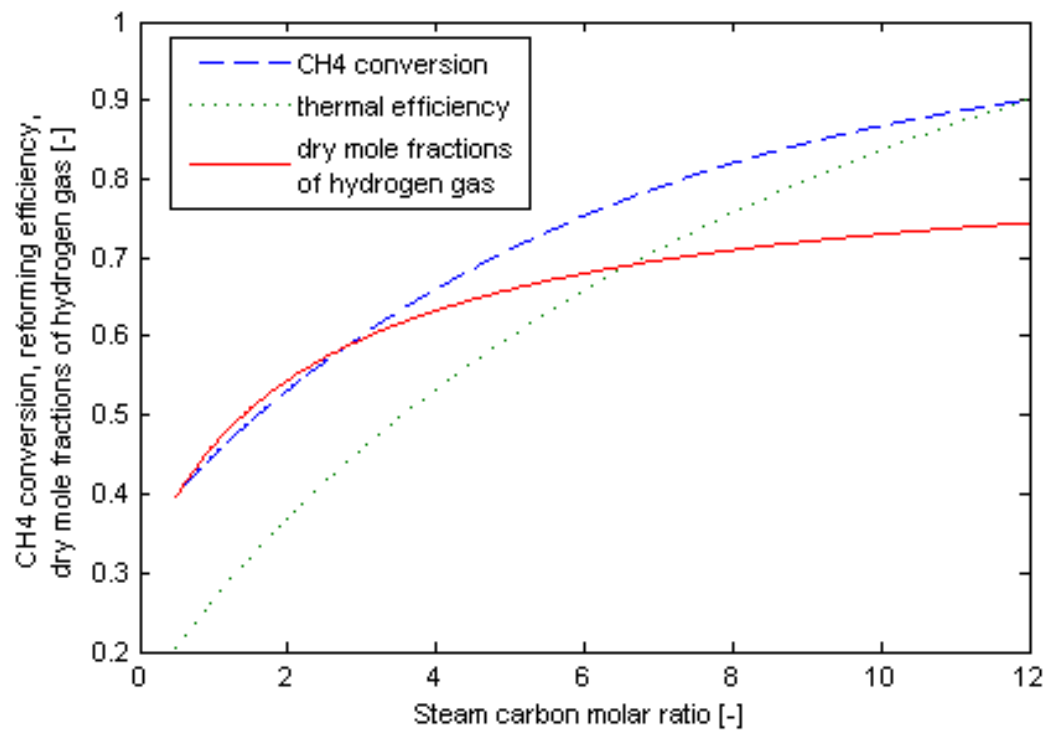

Figure 41: Simulation results for the ATR unit at constant temperature $500^{\circ} \mathrm{C}$ and $\mathrm{O} / \mathrm{C}=0.45$

according to the values in Table 22 so that the total inlet gas composition ratios became $\mathrm{S} / \mathrm{C}=1.2$ and $\mathrm{O} / \mathrm{C}=0.2$. Results from the simulation are shown in Figure 44 .

The results were compared to those from a similar simulation in literature [11]. The steady-state temperature in the reformer reaches $650^{\circ} \mathrm{C}$, which is very close to the corresponding value in the article [11]. Conversion of $\mathrm{CH}_{4}$ is slightly lower in Figure 44 than that in the article but within reasonable range. Utilization of $\mathrm{O}_{2}$ is close to 1 which is good since oxygen in the outlet composition leads to lower overall system efficiency. The reformer thermal efficiency is not calculated in a comparable way in the reference article [11], but is plotted in Figure 41, 42 and 44 for comparison between results from constant and dynamic temperature simulations. The steady-state mole fractions of the outlet gas from the simulation is presented in Table 23. Since there are only minor differences between these values and those listed in [11] the dynamic behavior of the reformer may be considered as accurate. 


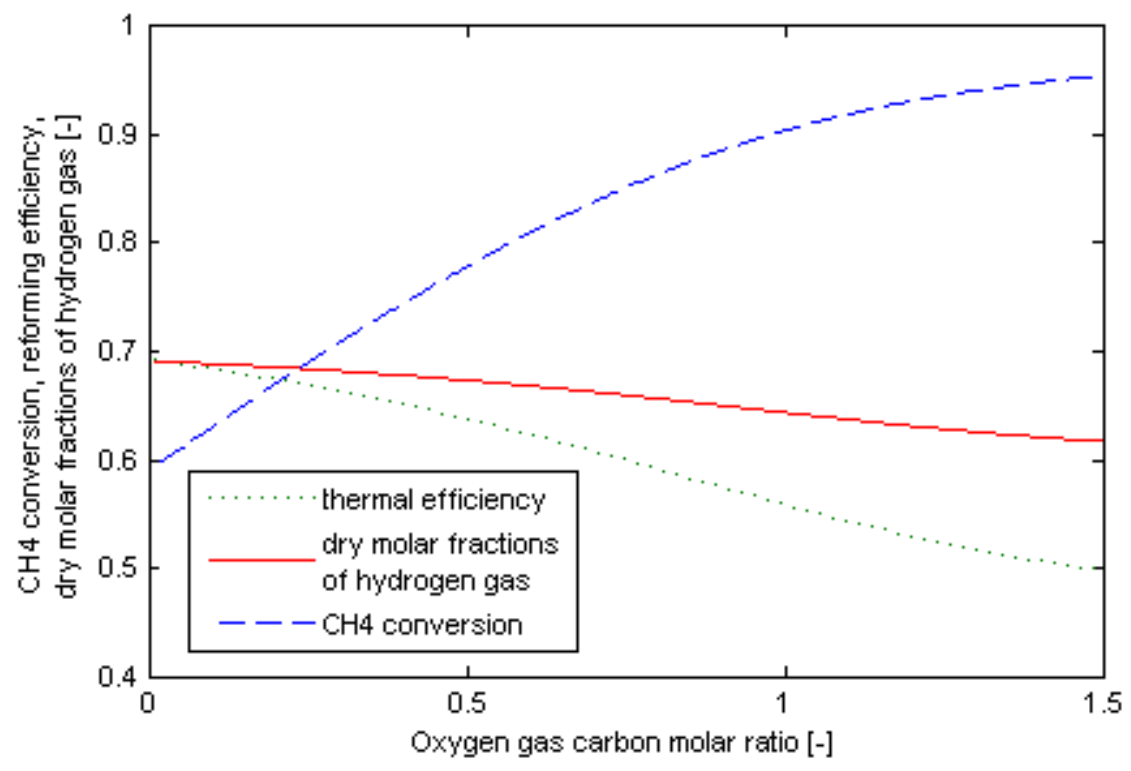

Figure 42: Simulation results for the ATR unit at constant temperature $500^{\circ} \mathrm{C}$ and $\mathrm{S} / \mathrm{C}=6$

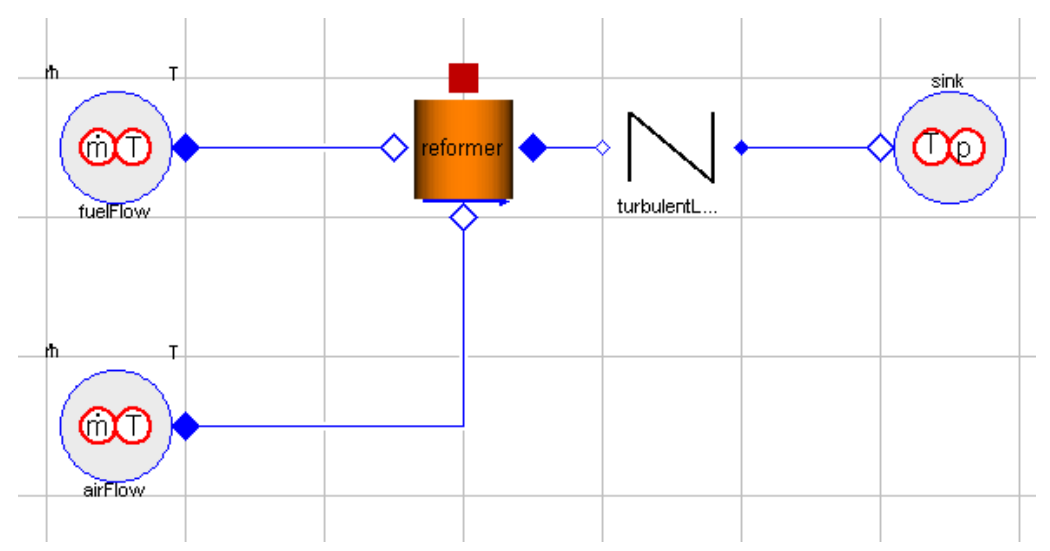

Figure 43: Experimental setup for validation of the autothermal reformer 
Reformer parameters

\begin{tabular}{lr}
\hline Volume & $0.0024 \mathrm{~m}^{3}$ \\
Initial temperature & $500{ }^{\circ} \mathrm{C}$ \\
Initial pressure & $1.5 \mathrm{bar}$ \\
Nominal mass flow rate & $9 \cdot 10^{-4} \mathrm{~kg} / \mathrm{s}$ \\
$L H V_{\mathrm{H}_{2}} 240 \mathrm{MJ} / \mathrm{kmol}$ & \\
LH $V_{\mathrm{CH} H_{4}} 800 \mathrm{MJ} / \mathrm{kmol}$ & \\
Sources & $500{ }^{\circ} \mathrm{C}$ \\
Temperature & $5.1 \cdot 10^{-4} \mathrm{~kg} / \mathrm{s}$ \\
Fuel mass flow rate & $3.9 \cdot 10^{-4} \mathrm{~kg} / \mathrm{s}$ \\
Air mass flow rate & \\
& \\
Inlet fuel composition ratios & 0.46 \\
\hline CH4/NG & 0.54 \\
H2O/NG & 0 \\
H2/NG & 0 \\
CO2/C & 0 \\
CO/C & \\
turbulentLoss & \\
\hline Nominal density & $1 \mathrm{~kg} / \mathrm{m}^{3}$ \\
Nominal pressure drop & $0.01 \mathrm{bar}$ \\
Sink & \\
\hline Pressure in sink &
\end{tabular}

Table 22: Parameters used in the dynamic validation of the autothermal reformer

\begin{tabular}{lr} 
Component & Mole fraction \\
\hline $\mathrm{H}_{2}$ & 0.2766 \\
$\mathrm{CH}_{4}$ & 0.1696 \\
$\mathrm{CO}$ & 0.0180 \\
$\mathrm{CO}_{2}$ & 0.0833 \\
$\mathrm{H}_{2} \mathrm{O}$ & 0.2453 \\
$\mathrm{~N}_{2}$ & 0.2055 \\
$\mathrm{O}_{2}$ & 0.0017
\end{tabular}

Table 23: Steady-state mole fractions in outlet gas 
(a) Utilization and reformer efficiency

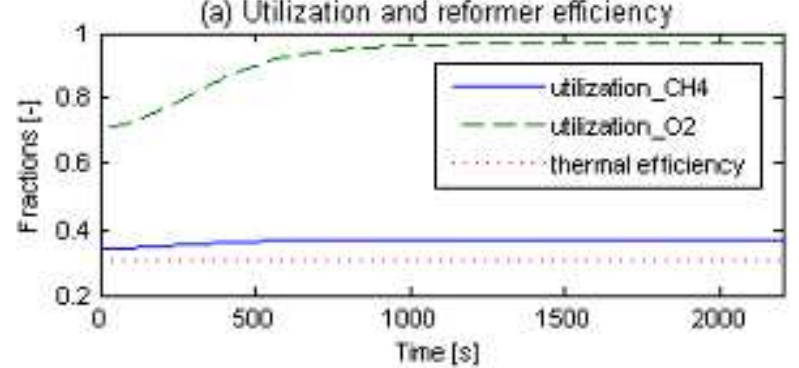

(b) Temperature in reformer

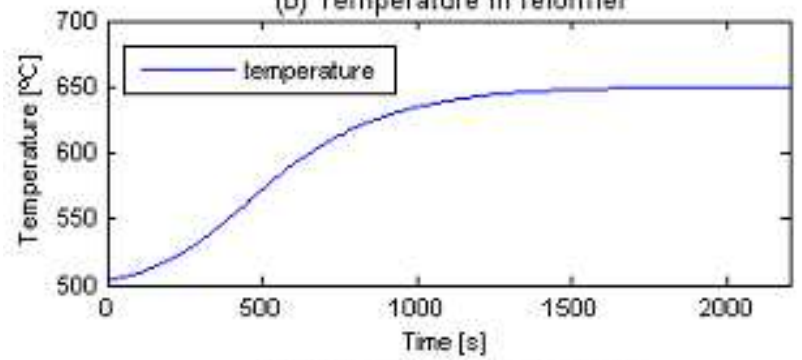

(c) Composition in outlet gas

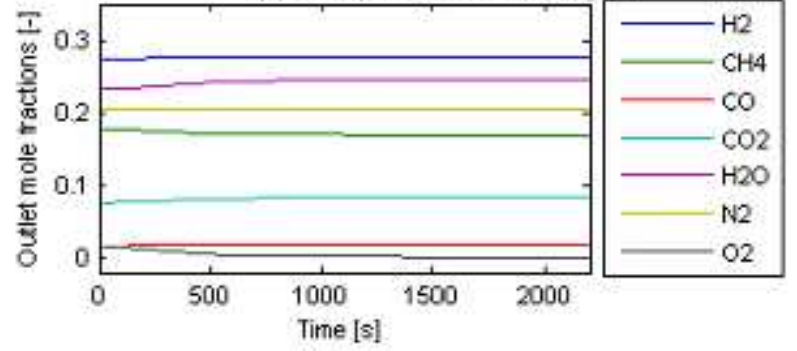

Figure 44: Simulation results for the ATR unit with $\mathrm{S} / \mathrm{C}=1.2$ and $\mathrm{O} / \mathrm{C}=0.2$ : (a) Utilization and thermal reforming efficiency in the reformer, (b) Temperature in the reformer, (c) Component mole fractions in the outlet gas. 


\subsubsection{Steam generator and fuel mixing}

The steam generator and fuel mixing models are verified by simulating the simple system shown in Figure 45. The steam generator is supplied with water and hot air at constant flow rates. The hot air leaves the steam generator to a sink at constant pressure and the generated steam is fed to the mixing volume where fuel and steam are mixed. Fuel is supplied at constant flow rate to the mixing volume and the volume outlet is fed to a sink at constant pressure. For this simulation the parameters presented in Table 24 are used.

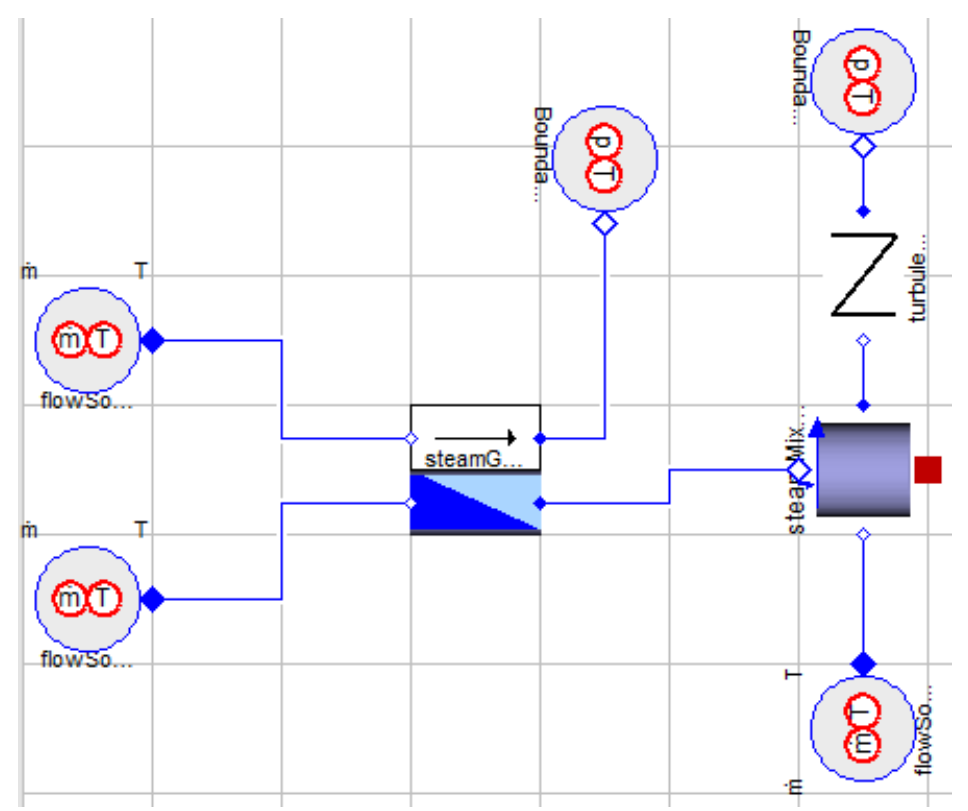

Figure 45: Experiment setup for steam generator and fuel mixing verification

The geometry parameters were chosen to represent a steam generator of reasonable size. The temperature of the hot air was chosen according to the simulation results of the catalytic burner presented in chapter 5.6.3. The fuel composition is close to pure methane, with some additional carbon dioxide, which is assumed to be the outlet composition of a process where longer carbon chains have been reformed.

In Figure 46(a) simulation results for the temperature are presented. The steam itself reaches a high temperature close to $800^{\circ} \mathrm{C}$, whereas the temperature of the steam generator thermal mass reaches $620^{\circ} \mathrm{C}$. The steam is mixed with natural gas at a lower temperature and this mixture reaches a temperature of $600^{\circ} \mathrm{C}$. Figure $46(\mathrm{~b})$ shows the pressure in the mixing volume. The pressure increases only slightly.

The composition of the fuel mixture is shown in Figure 47. In this case the mixture has a high steam content, which is a requirement for the autothermal reformer to operate properly. The methane content is roughly $25 \%$ and there is 
also a small amount of carbon dioxide.

Steam generator

\begin{tabular}{lr}
\hline Number of parallel flow channels & 4 \\
Length & $0.4 \mathrm{~m}$ \\
Cross section area & $0.005 \mathrm{~m}^{2}$ \\
Circumference & $0.2 \mathrm{~m}$ \\
Outside wall area & $0.03 \mathrm{~m}^{2}$ \\
& \\
Mixing volume & \\
\hline Volume & $0.01 \mathrm{~m}^{3}$ \\
Initial temperature & $327^{\circ} \mathrm{C}$ \\
Initial pressure & $1.1 \mathrm{bar}$
\end{tabular}

Pressure drop

Nominal density

Nominal pressure drop

Nominal flow rate

$1 \mathrm{~kg} / \mathrm{m}^{3}$
$0.01 \mathrm{bar}$
$10^{-4} \mathrm{~kg} / \mathrm{s}$

Sources

Hot air temperature

$1227{ }^{\circ} \mathrm{C}$

Hot air flow rate

$4 \cdot 10^{-3} \mathrm{~kg} / \mathrm{s}$

Water temperature

$25^{\circ} \mathrm{C}$

Water flow rate

Fuel temperature

Fuel flow rate

Fuel composition

$3 \cdot 10^{-4} \mathrm{~kg} / \mathrm{s}$

$200{ }^{\circ} \mathrm{C}$

$1 \cdot 10^{-4} \mathrm{~kg} / \mathrm{s}$

$98 \% \mathrm{CH}_{4}, 2 \% \mathrm{CO}_{2}$

Sinks

Pressure of all sinks

1.013 bar

Table 24: Parameters used in the steam generator and fuel mixing verification 

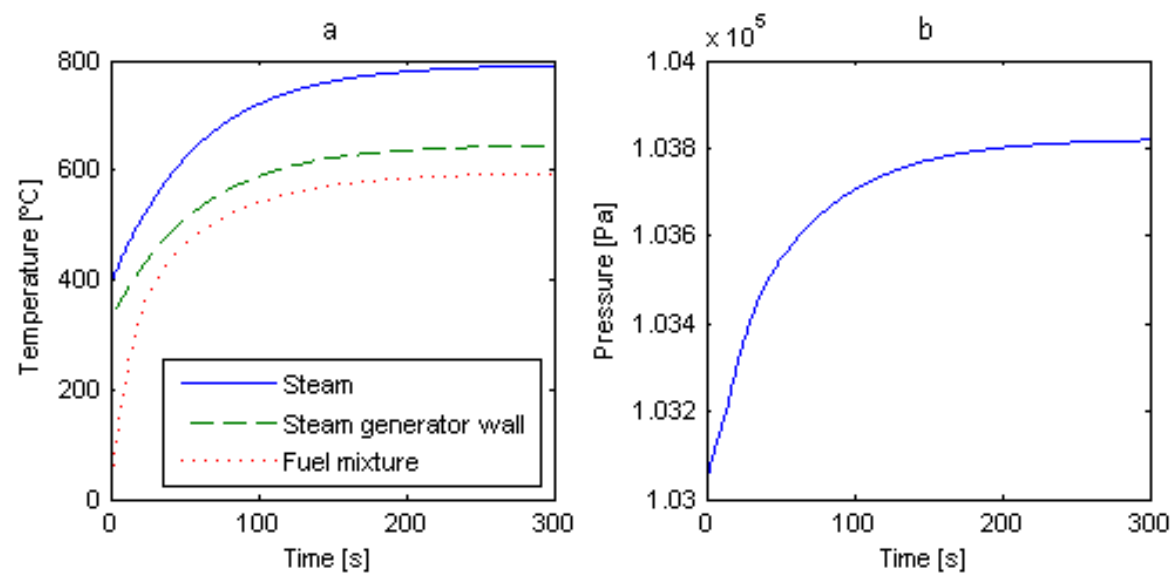

Figure 46: Simulation results for: (a) temperature of the steam, steam generator wall and fuel mixture, (b) pressure in the mixing volume

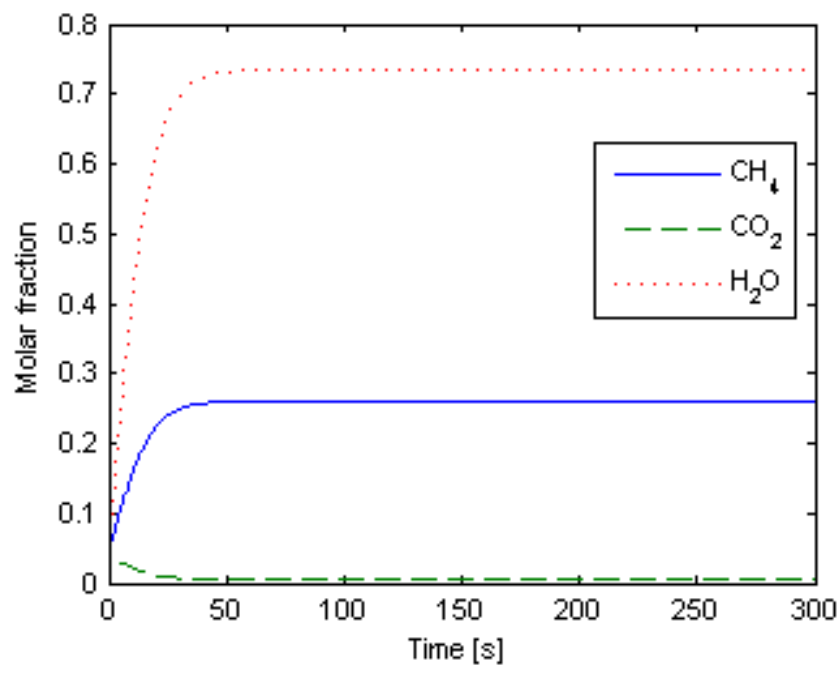

Figure 47: Simulation results for the fuel mixture composition 


\subsubsection{Catalytic burner}

The catalytic burner is verified by simulating the simple setup shown in Figure 48. The burner is placed in the middle of the setup and has a constant air supply connected to it. Fuel is supplied from a sink with constant composition and temperature at a flow rate increasing linearly over time. The burner exhaust is fed through at turbulent pressure drop model and then to a sink constrained to constant pressure. Ignition pulses are generated by a boolean pulse generator block.

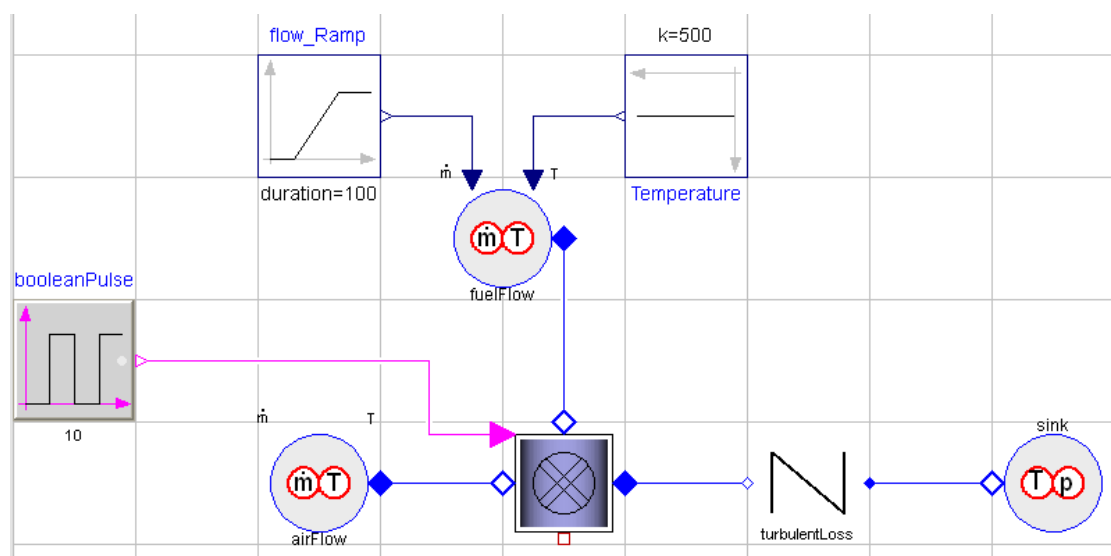

Figure 48: Experiment setup for verification of the catalytic burner

\begin{tabular}{lr} 
Burner & \\
\hline Volume & $0.01 \mathrm{~m}^{3}$ \\
derscale & 0.95 \\
Initial pressure & $1.1 \mathrm{bar}$ \\
Initial temperature & $120^{\circ} \mathrm{C}$ \\
& \\
Air feed & $2 \cdot 10^{-3} \mathrm{~kg} / \mathrm{s}$ \\
\hline Flow rate & $100^{\circ} \mathrm{C}$ \\
Temperature & \\
Fuel feed & $0{\text { to } 2 \cdot 10^{-3} \mathrm{~kg} / \mathrm{s}}^{5}$ \\
Flow rate & $500^{\circ} \mathrm{C}$ \\
Temperature & \\
Composition & $23 \% \mathrm{H}_{2}, 1.7 \% \mathrm{CH}_{4}, 3.1 \% \mathrm{CO}$, \\
& $5.9 \% \mathrm{CO}_{2}, 44 \% \mathrm{H}_{2} \mathrm{O}, 22 \% \mathrm{~N} 2$
\end{tabular}

Table 25: Parameters used in the verification of the burner model

The parameters used in this simulation are given in Table 25. The fuel composition is the same as used in [11] for burner model validation and the flow is ramped during 100 seconds. Ignition signals are given to the burner every 10th second with the first pulse at 10 seconds after start-up. 
a

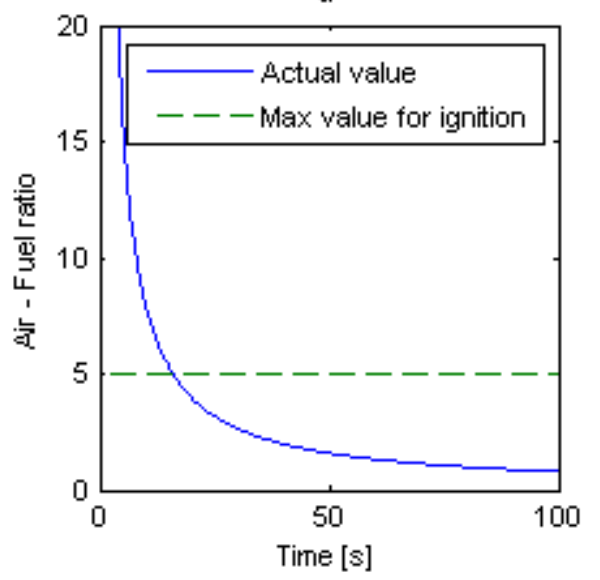

b

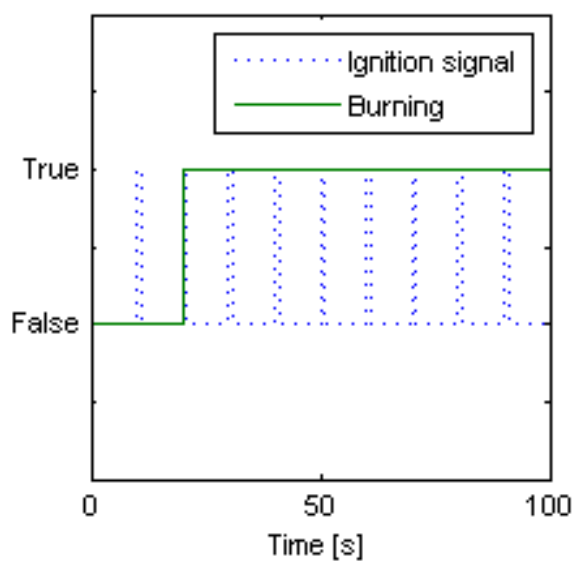

Figure 49: Simulation results for: (a) Air to fuel ratio, (b) Burner state

Figure 49(a) shows the air to fuel ratio in the burner during the simulation and Figure 49(b) shows the ignition signal and burner state. The air to fuel ratio is defined as the ratio between the oxygen inlet flow and the oxygen flow required for complete combustion of the inlet fuel. For ignition to occur at a given ignition signal the air to fuel ratio must not be larger than a maximum value, which is also shown in Figure 49(a). When the first ignition pulse occurs after 10 seconds this is not the case and therefore the burner remains in its mixing state. At $T=20 \mathrm{~s}$ there is enough fuel available for ignition to occur and thus the burner switches to its burning state, as shown in Figure 49(b).

(a) Temperature

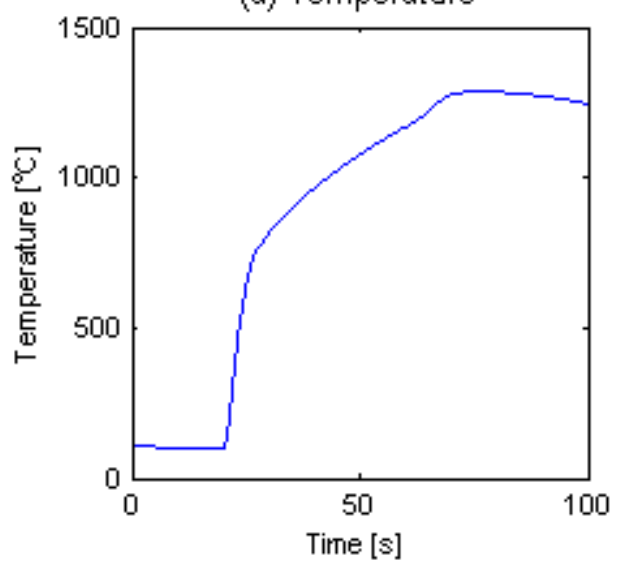

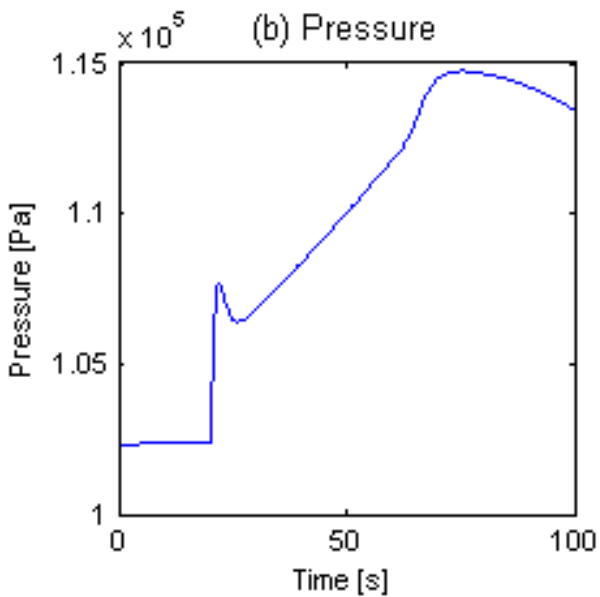

Figure 50: Simulation results for: (a) Temperature of the burner flue gas, (b) Pressure of the burner flue gas

In Figure 50 the temperature and pressure of the burner flue gas are shown. Before ignition both the temperature and pressure vary only slightly as ex- 
pected, and when the burner is ignited both of them increase rapidly. The temperature reaches a maximum value of $1290^{\circ} \mathrm{C}$ but decreases towards the end of the simulation due to a thicker fuel mixture which decreases the combusted fuel fraction. The pressure shows a curve similar to that of the temperature and has a maximum value of 1.147 bar.

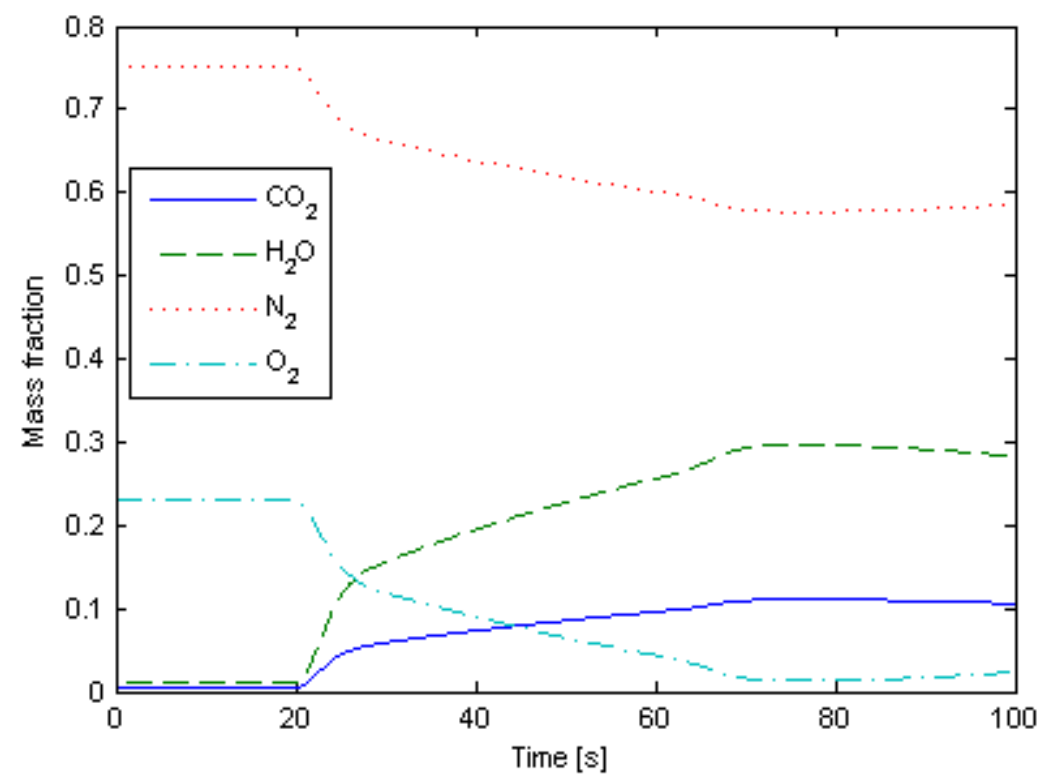

Figure 51: Mass fractions of the flue gas components

Figure 51 shows the mass fractions of the components in the burner flue gas. It can be seen that when combustion occurs the oxygen content decreases and the carbon dioxide and steam content increases due to the combustion. Also the nitrogen content decreases due to the overall mass increase when components are added to the flue gas from the fuel. The nitrogen itself is not active in any reaction. 


\subsection{System model validation}

\subsubsection{Hotbox validation}

The hotbox model is verified using the setup shown in Figure 52. Constant flow rates and compositions are supplied to each feed port and the resulting fuel temperature and composition is investigated.

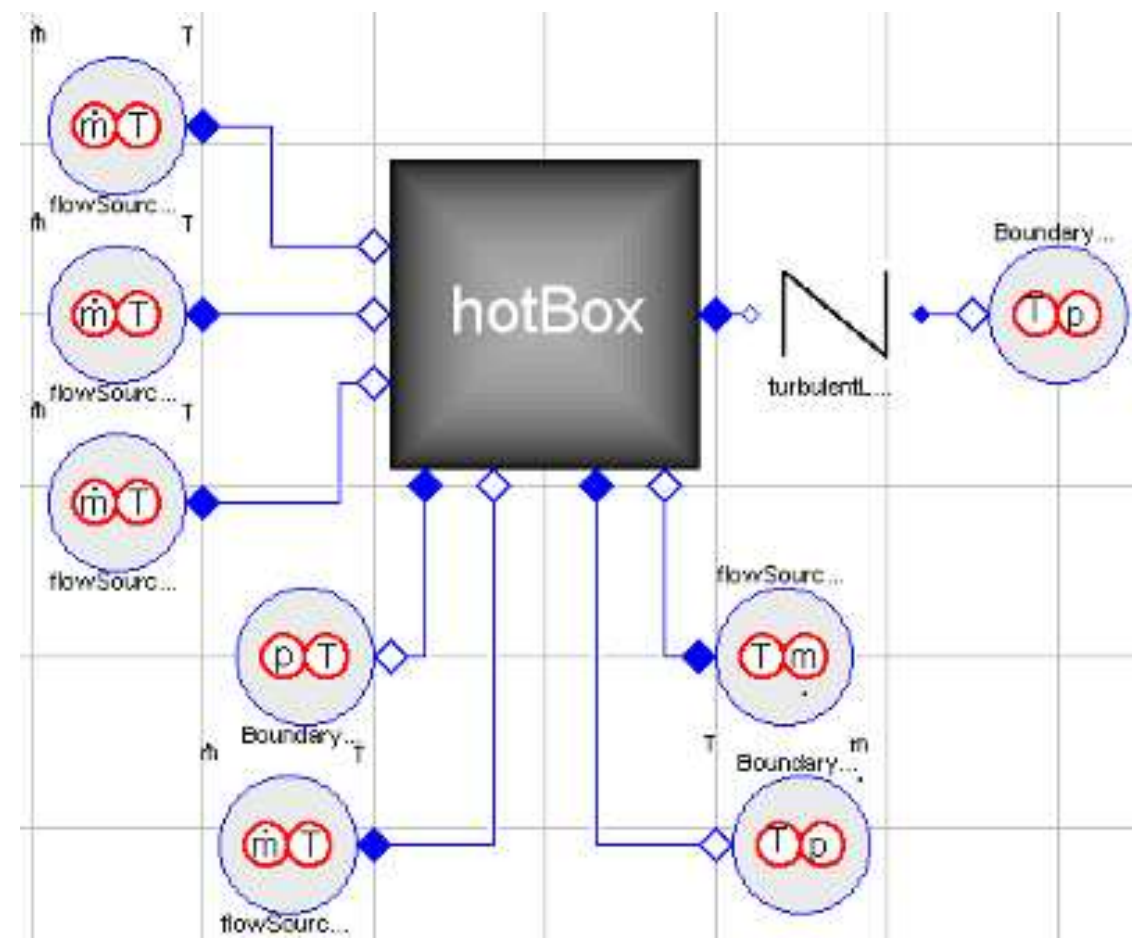

Figure 52: Experiment setup for verification of the hotbox model

The parameters given in Table 26 are found to give a fuel outlet suitable to feed the stack with. The results from a 1000 second simulation with these parameters are presented in the following figures.

Figure 53 shows the temperatures for some components of the hotbox. The outlet temperature is the temperature of the fuel produced in the ATR unit and is $780^{\circ} \mathrm{C}$ in steady-state in this simulation. The temperature in the steam mixing volume reaches $520^{\circ} \mathrm{C}$ in steady-state and the temperature of the steam produced in the steam generator is $280^{\circ} \mathrm{C}$ in steady-state.

In Figure 54 the resulting mole fractions of the important fuel components are presented. In this simulation the hydrogen mole fraction is 0.27 in steadystate, the methane mole fraction is 0.16 and the steam mole fraction is 0.25 . The steady-state values for the components not presented in the figure are 0.018 for carbon monoxide, 0.089 for carbon dioxide, 0.21 for nitrogen gas and 0.0004 for oxygen gas. 


\begin{tabular}{lr} 
Hotbox initialization & \\
\hline All temperatures & $400^{\circ} \mathrm{C}$ \\
All pressures & $1.1 \mathrm{bar}$ \\
& \\
Flow sources & $4 \cdot 10^{-4} \mathrm{~kg} / \mathrm{s}$ \\
\hline Air flow rate & $100^{\circ} \mathrm{C}$ \\
Air temperature & $2.8 \cdot 10^{-4} \mathrm{~kg} / \mathrm{s}$ \\
Water flow rate & $25^{\circ} \mathrm{C}$ \\
Water temperature & $2.2 \cdot 10^{-4} \mathrm{~kg} / \mathrm{s}$ \\
Natural gas flow rate & $300^{\circ} \mathrm{C}$ \\
Natural gas temperature & $1.4 \cdot 10^{-3} \mathrm{~kg} / \mathrm{s}$ \\
Hot gas flow rate & $1200^{\circ} \mathrm{C}$ \\
Hot gas temperature & $5 \cdot 10^{-4} \mathrm{~kg} / \mathrm{s}$ \\
Hot exhaust flow rate & $850^{\circ} \mathrm{C}$ \\
Hot exhaust temperature & \\
Boundary conditions & $1.013 \mathrm{bar}$
\end{tabular}

Table 26: Parameters used in the verification of the hotbox model

In Figure 55(a) the methane and oxygen utilizations of the ATR unit are presented. The utilization of oxygen is very close to one, indicating that almost all oxygen is consumed in the ATR unit. This is desired as oxygen in the fuel may contribute to combustion of hydrogen without producing any energy to the external electric circuit. The methane utilization is 0.4 , which is close to the result in [11].

Finally the steam carbon ratio and oxygen carbon ratio are presented in Figure 55 (b). These ratios are explicitly given by the inlet flows and thus they are constant during the simulation. The inlet flows used in this simulation corresponds to a steam carbon ratio of 1.19 and an oxygen carbon ratio of 0.22 .

The hotbox model is quite sensitive to changes in the flow rates and temperatures. All inlets must be balanced to give a good outlet composition and keep temperatures within a reasonable range. The effects of varying individual flows were investigated with the following results:

- The air flow to the ATR unit affects the available amount of oxygen. An increased flow supplies more oxygen and thereby increases the rate of the total combustion of methane. This gives an increased temperature which will cause the produced amount of hydrogen to decrease. With the parameters given in Table 26 and an air flow rate of $6 \cdot 10^{-4} \mathrm{~kg} / \mathrm{s}$ the temperature reaches $1000^{\circ} \mathrm{C}$ and the fuel hydrogen content is decreased. The air flow rate can not be to low either, as some total combustion of methane is required to keep the temperature up. With a flow rate lower than $3.9 \cdot 10^{-4} \mathrm{~kg} / \mathrm{s}$ together with the parameters given in Table 26 the system can not be simulated. The temperature of the air is not as important as the flow rate. A higher temperature gives a slightly higher fuel temperature and vice versa. 


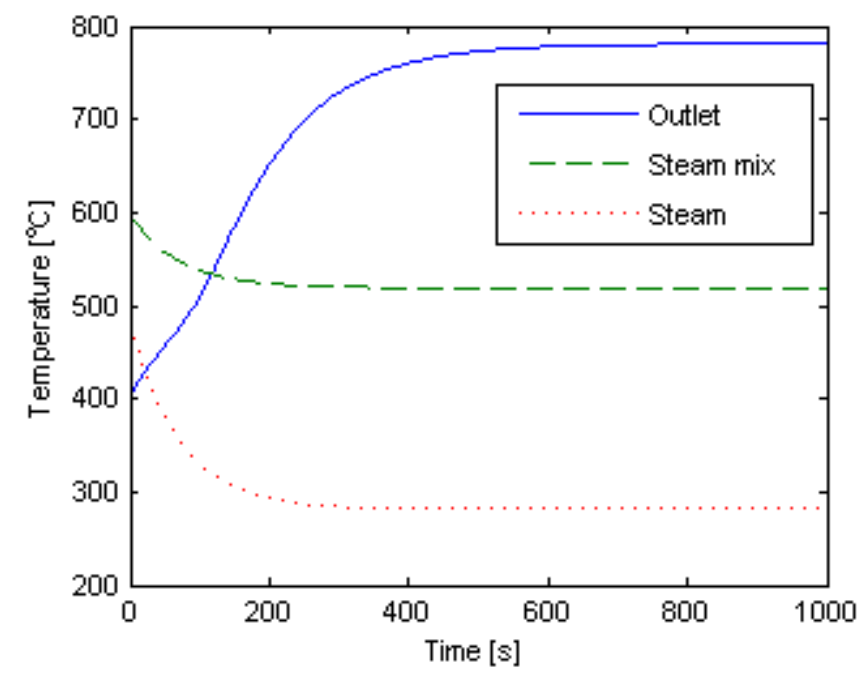

Figure 53: Simulation results of the hotbox temperatures

- The water flow rate affects the important steam carbon ratio in the ATR unit. When the flow rate is increased above $3.3 \cdot 10^{-4} \mathrm{~kg} / \mathrm{s}$ and all other flows as given in Table 26 are used the amount of total combustion is decreased to such an extent that the temperature is to low for the reforming reactions to occur. A lowered water flow rate gives an increased rate of total combustion which results in a higher temperature and eventually a decreased hydrogen production rate. A water flow rate of $2 \cdot 10^{-4} \mathrm{~kg} / \mathrm{s}$ results in a fuel outlet temperature of $1000^{\circ} \mathrm{C}$.

- The flow rate of natural gas affects both the steam carbon ratio and the oxygen carbon ratio and is thereby a critical parameter. An increased flow rate was found to lower the outlet fuel temperature and with flow rates greater than $4 \cdot 10^{-4} \mathrm{~kg} / \mathrm{s}$ the temperature became to low for a good hydrogen production rate. Flow rates lower than $1.9 \cdot 10^{-4} \mathrm{~kg} / \mathrm{s}$ causes simulation errors.

- The flow rate of hot gas to the steam generator must be large enough to supply the heat required for steam generation. The flow rate also affects the temperature of the steam and thereby the temperature in the ATR unit. With flow rates lower than $1 \cdot 10^{-3} \mathrm{~kg} / \mathrm{s}$ the temperature became to low for good hydrogen production rate. An increased flow rate cause the temperature of the outlet fuel to increase and it reaches $1000^{\circ} \mathrm{C}$ at a hot gas flow of $4 \cdot 10^{-3} \mathrm{~kg} / \mathrm{s}$.

- The flow rate of the stack exhaust heating the air prior to entering the ATR unit can be changed relatively much without any significant problems. The flow could be varied between $1 \cdot 10^{-4} \mathrm{~kg} / \mathrm{s}$ and $1.2 \cdot 10^{-3} \mathrm{~kg} / \mathrm{s}$ together with the parameters in Table 26. An increased flow rate gives a slightly increased temperature of the outlet fuel. 


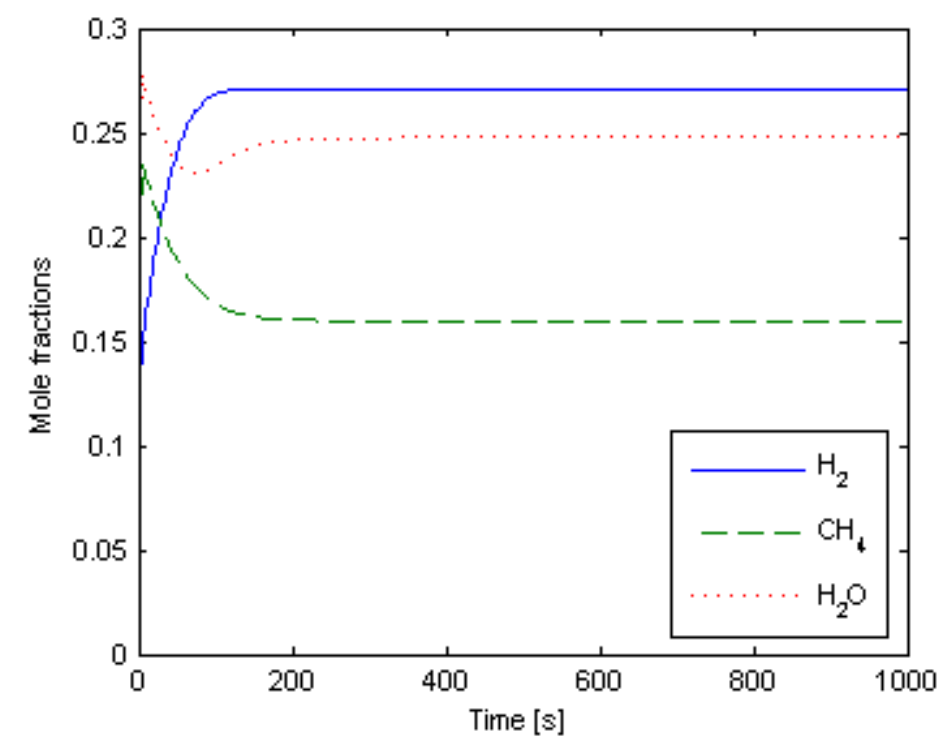

Figure 54: Simulation result of the hotbox fuel outlet composition
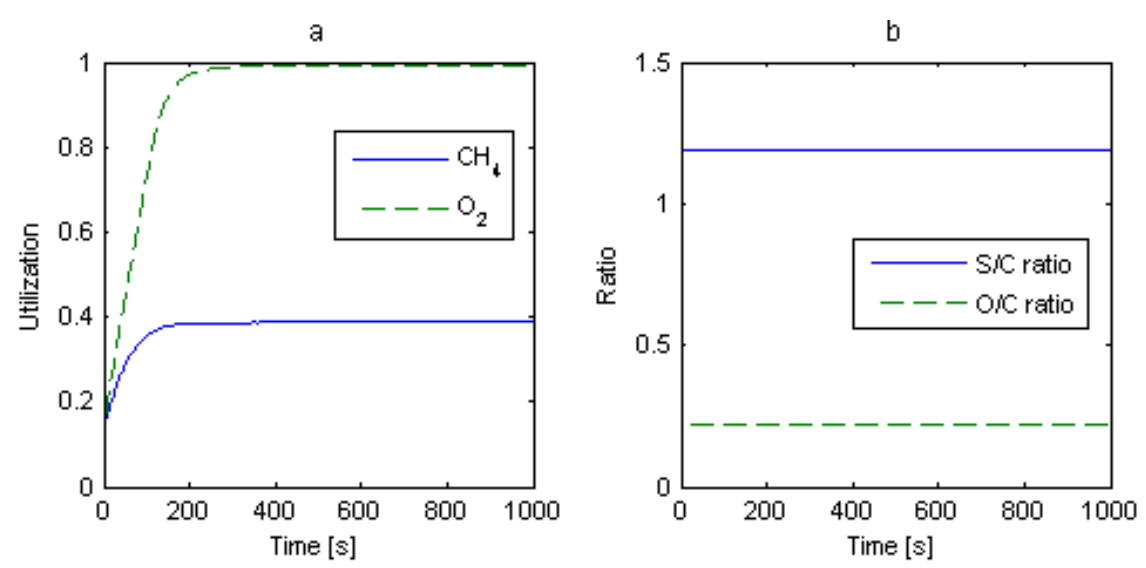

Figure 55: Simulation results of: (a) The ATR unit methane and oxygen utilization, (b) The steam carbon and oxygen carbon ratios of the ATR units 


\subsubsection{System validation}

The system in Figure 15 is simulated for 45 minutes in order to show its performance. The inlet flow rates of air, natural gas and water to the hotbox are chosen so that a steam carbon ratio of 1.2 and a oxygen gas carbon ratio of 0.2 is achieved in the autothermal reformer. In addition the flow rates are chosen so that the total inlet flow rate to the hotbox becomes $1.035 \cdot 10^{-3} \mathrm{~kg} / \mathrm{s}$. The stack anode is fed with the outlet gas from the hotbox and the cathode is fed with air at a constant flow rate of $2 \cdot 10^{-2} \mathrm{~kg} / \mathrm{s}$. The 50 cells of the stack are divided between three substacks connected in series. The first and the last substack contains 10 cells each and the second substack contains 30 cells. The cells are modeled by the complete electrochemical model presented in chapter 2.3 and the cell geometry given in Table 20 is used. The stack temperature is initialized at $800^{\circ} \mathrm{C}$ and the nominal pressure drop parameters in the manifold volumes of the stack are set to $100 \mathrm{~Pa}$. Initial pressures in the components are set to decreasing values starting from 1.56 bar in the hotbox in order to get positive flows during start-up. The exernal current generator connected to the top of the stack is set to a constant value of 150 A. This corresponds to a current density of $4155 \mathrm{~A} / \mathrm{m}^{2}$. Results from the simulation are presented in Figures $56-60$.

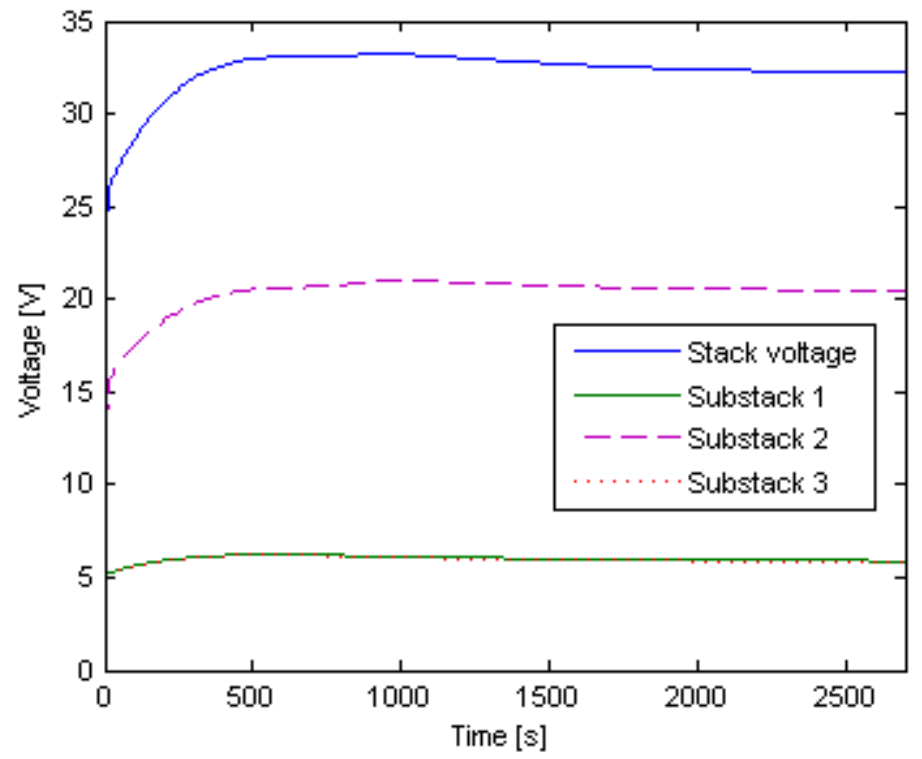

Figure 56: Simulation results for stack and substack voltages

The voltages over the complete stack and over the individual substacks are shown in Figure 56. The total stack voltage reaches a steady-state value of $32.26 \mathrm{~V}$. This corresponds to a cell voltage of $0.6452 \mathrm{~V}$, which complies to the results from the stack validation in section 5.5. As in the results from the stack validation, the highest voltage per cell is obtained in the second substack due to a higher temperature than in the other substacks. Temperatures in the different substacks are presented in Figure 57(a). The steady-state temperatures are $1009^{\circ} \mathrm{C}$ in substack $1,1064^{\circ} \mathrm{C}$ in substack 2 and $1018^{\circ} \mathrm{C}$ in substack 3 . The 
temperature difference between the top and the bottom substacks can be explained by the pressure drop in the manifold volumes. The hydrogen utilization of the substacks are shown in Figure 57(b). The values are slightly larger than the corresponding values in the stack validation in section 5.5. This is a consequence of a slightly different composition of the reformed fuel fed to the stack anode, compared to that in the stack validation section.
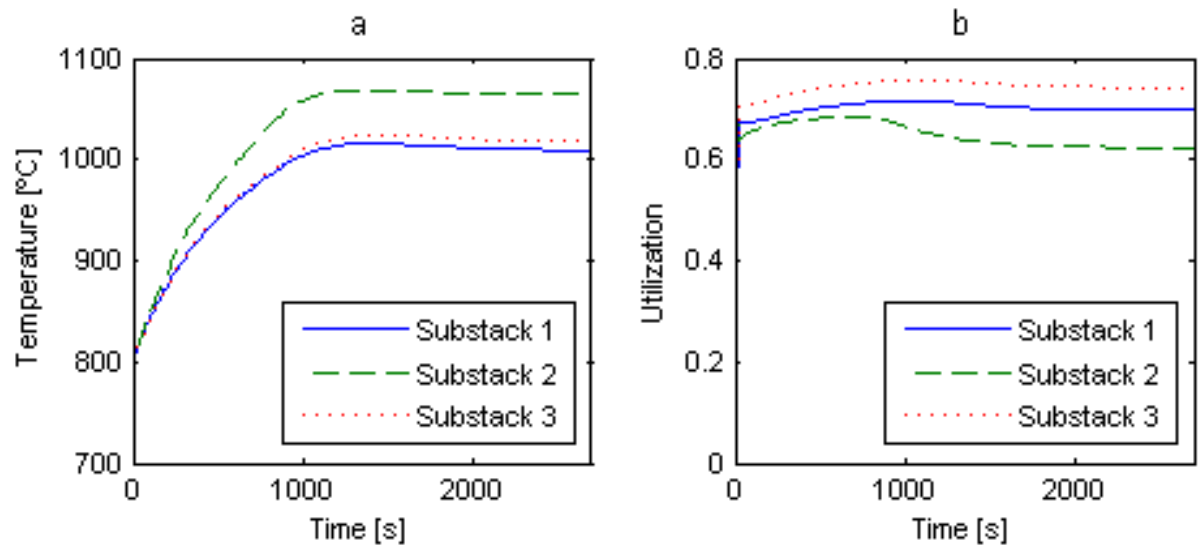

Figure 57: Simulation results for: (a) Substack temperatures, (b) Substack utilizations

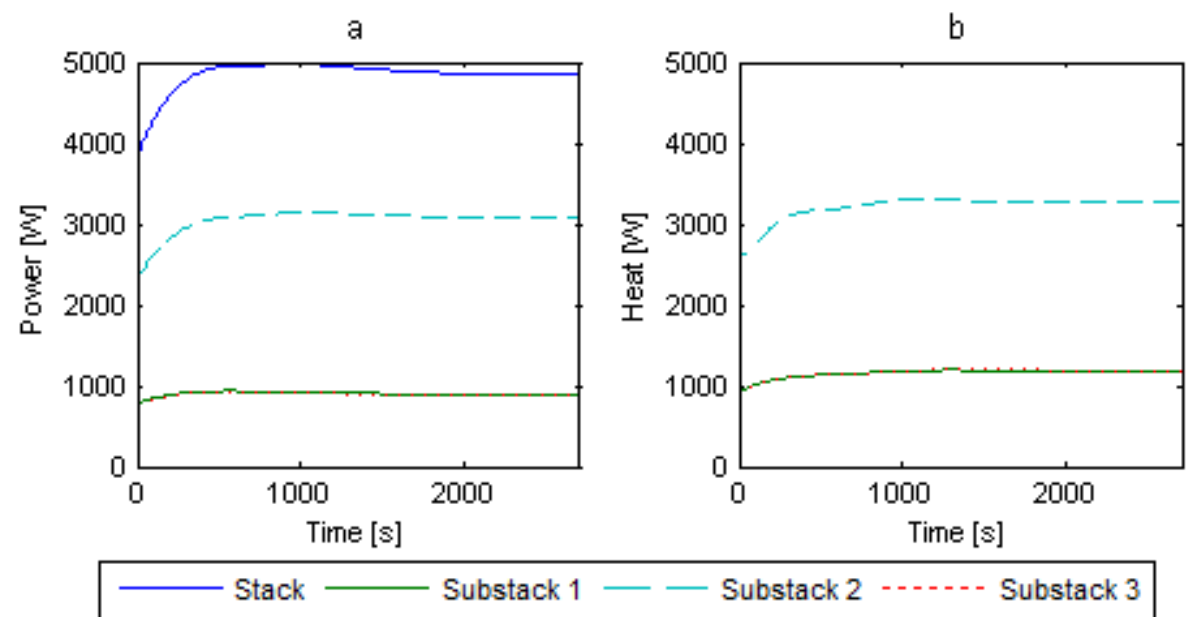

Figure 58: Simulation results for: (a) Stack and substack power generation, (b) Substack heat production

The power generation of the complete stack and the individual substacks are presented in Figure 58(a). The total power production of the system reaches $4838 \mathrm{~W}$ in steady-state. The heat production in the substacks are shown in Figure 58(b). 
a

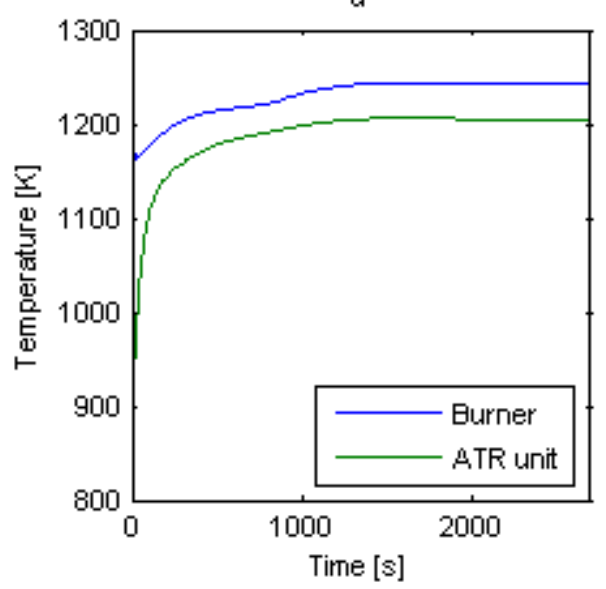

b

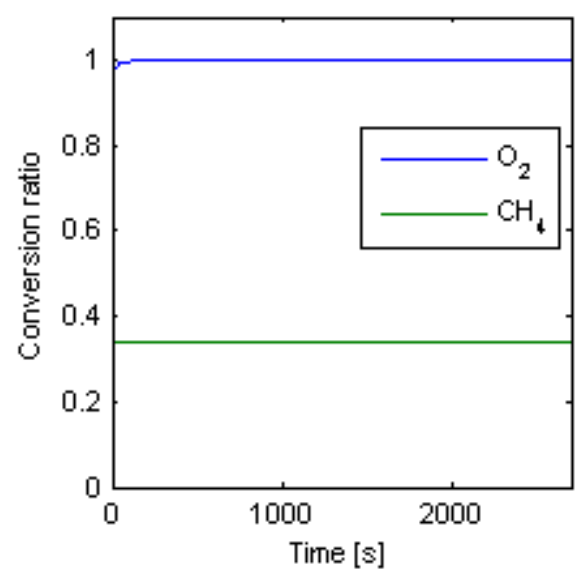

Figure 59: Simulation results for: (a) Burner and ATR unit temperature, (b) ATR conversion ratio of methane and oxygen

It is important to keep record of the temperature in the autothermal reformer since the outlet gas from the reformer is fed to the stack anode. The ATR temperature is shown in Figure 59(a) together with the temperature of the burner. The temperature in the ATR unit reaches $931^{\circ} \mathrm{C}$ in steady-state, and the burner reaches $970^{\circ} \mathrm{C}$. It is important to have a high temperature in the burner since the heat in the exhaust gas is used for steam generation in the hotbox model. Conversion ratios of oxygen gas and methane in the reformer are presented in Figure 59(b). $\mathrm{O}_{2}$ conversion is close to 1, which is good, as oxygen in the fuel lowers the overall system performance. The methane utilization is 0.34 , which is slightly lower than the corresponding value from the autothermal reformer validation in section 5.6.1. The lower conversion of methane is also reflected in the outlet composition from the reformer, presented in Figure 60. Less methane has been converted due to the higher temperature in the reformer, compared to the results presented in Table 23. However, the differences between the compositions are small, and does not affect the overall system performance very much. 


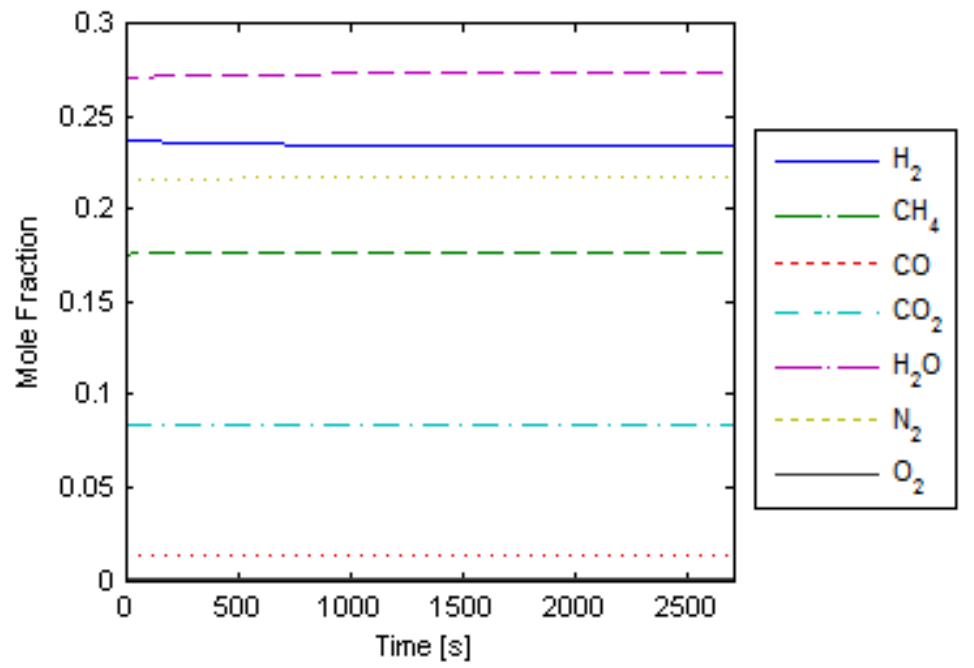

Figure 60: Simulation results for the ATR outlet fuel composition 


\section{Conclusions}

\subsection{Dymola and Modelica}

The Dymola software has both positive and negative aspects that have been experienced in the course of this study. The object oriented modeling method is very suitable for any models where reuse of code is desirable. In this project many model components are used more than once in the overall system model. One example is the medium models which are used in all models of objects containing a medium. Another example is the heat exchanger models, as three applications are included in the complete system model. The object oriented structure also allows specific parts of a model to be easily replaced. In this study two cell models were implemented, one with ohmic losses only and one with complete polarization effects. Since these two models are objects of the same type, the stack and system models can use any one of them simply by changing a parameter.

The Modelica language is suitable for most types of system models where the system behaviour is described by a system of differential and algebraic equations. The equation based modeling approach gives a lot of flexibility.

There are also negative aspects, however. Dymola is not very suitable for simulating systems of partial differential equations, as done by finite element and finite volume methods. Other software, such as COMSOL Multiphysics, can automatically perform the discretization of such a problem, whereas in Dymola the user must perform the discretization by stating the equations directly.

Another issue for new users of Modelica is the requirement for model packages. Modelica includes an open model package with basic models for electrical, mechanical and thermal calculations. This package is quite limited in terms of function, but the user may develop or purchase extra model packages. In this project there were extra models for fluid systems available, i.e. the Modelon CombiPlant library and other project models.

As a tool Dymola is probably most useful for control applications where the overall system behaviour is more important than the details. Dymola offers many possibilites for control applications, such as hardware in the loop simulation.

\subsection{Cell modeling}

Two different cell models were implemented in the course of this study, based on data presented in the open literature. The first model includes ohmic loss only, which is a highly simplified model compared to the underlying physics. The second one includes a full polarization model, and mainly consists of equations describing the actual underlying physics. These two models have different objectives and applications, and different requirements in terms of computing power.

The full polarization model may be useful in the design stage of a fuel cell, 
as it can increase the understanding of the physical processes in the fuel cell. For control applications, however, it is probably too detailed. It includes effects that changes the cell voltage less than a few hundredths of a volt. On the other hand other parameters, such as the number of discretizations, change the cell voltage in the same order of magnitude.

The simplified model on the other hand is not very physically correct, as the only voltage loss included is the ohmic loss, and is calculated from an empirical correlation which is temperature dependent only. Nevertheless such a model may be useful when studying a fuel cell after the empirical expression has been fitted to the voltage - current characteristics and temperature dependence of that specific cell within a range of operating conditions. For simulations of the complete system this cell model may be accurate enough. Also for realtime applications the complete polarization model is probably too complex to simulate in real-time, and the simplified model or even table based models may be used.

\subsection{System modeling}

The system modeling is a strong aspect of Dymola and Modelica. The object oriented structure and support for acausal models make the system modeling relatively simple. The components are connected in the model as they are physically. Some problems were still experienced during the system model development. As the system includes more components, more time must be spent on choosing proper initial conditions. The first stage of the simulation is to solve the initialization problem, and as the system grows this becomes harder for the non-linear solver to handle. Therefore more care must be taken to choose good initial values for each component.

Early in the project there were also some extra work related to the connections between components. For all connectors for mass flow, the relatively new stream connector concept is used. This caused some problems as some previously developed models had to be modified to support the stream connectors.

\subsection{Suggestions for future work}

Two areas have been identified where further work could be performed in the future. The first area is to adapt the model to describe other types of fuel cells than SOFCs. The single cell model is quite general and could probably well describe other types of fuel cells if the parameters were chosen accordingly. The fuel pre-processing would probably have to be improved, as lower temperature fuel cells are more sensitive to fuel inlet composition.

The second area is to improve the system model. This would require more extensive descriptions of fuel cell systems, and more measurement data to validate the model. Unfortunately no published measurement data have been found for entire fuel cell systems. 


\section{References}

[1] J. Larminie and A. Dicks. Fuel Cell Systems Explained, Second Edition. Wiley, 2003. ISBN 0-470-84857-X.

[2] M. Kemm. Dynamic Solid Oxide Fuel Cell Modelling for Non-steady State Simulation of System Applications. PhD thesis, Division of Thermal Power Engineering, Lund University, 2006.

[3] S.H. Chan, H.K. Ho, and Y. Tian. Modelling of simple hybrid solid oxide fuel cell and gas turbine power plant. Journal of Power Sources, 109:111$120,2002$.

[4] http://www.modelica.org, October 2009.

[5] http://www.3ds.com/products/catia/portfolio/dymola, October 2009.

[6] Dymola user manual. Volume 1, Document version 5, 2009.

[7] http://trac.edgewall.org/.

[8] http://subversion.tigris.org/.

[9] E. Maguire, B. Gharbage, F.M.B. Marques, and J.A. Labrincha. Cathode materials for intermediate temperature SOFCs. Solid State Ionics, 127:329335,2000 .

[10] H. Gao, J. Liu, H. Chen, S. Li, T. He, Y. Ji, and J. Zhang. The effect of Fe doping on the properties of SOFC electrolyte YSZ. Solid State Ionics, 179:1620-1624, 2008.

[11] J. Saarinen, M. Halinen, J. Ylijoki, M. Noponen, P. Simell, and J.Kiviaho. Dynamic model of $5 \mathrm{~kW}$ SOFC CHP test station. Journal of Fuel Cell Science and Technology, 4:397-405, 2007.

[12] P. Costamagna, A. Selimovic, M. Del Borghi, and G. Agnew. Electrochemical model of the integrated planar solid oxide fuel cell (IP-SOFC). Chemical Engineering Journal, 102:61-69, 2004.

[13] U.G. Bossel. Facts \& Figures, Final Report on SOFC data. Technical report, IEA, Swiss Federal Office of Energy, Berne, 1992.

[14] S.H. Chan, K.A. Khor, and Z.T. Xia. A complete polarization model of a solid oxide fuel cell and its sensitivity to the change of cell component thickness. Journal of Power Sources, 93:130-140, 2001.

[15] AirConditioning Library Users Guide. Version 1.6, Modelon AB, 2008.

[16] J. Yuan, Y. Huang, B. Sundén, and W. G. Wang. Analysis of parameter effects on chemical reaction coupled transport phenomena in SOFC anodes. Heat Mass Transfer, 45:471-484, 2009.

[17] Y. Cengel and M. Boles. Thermodynamics: An Engineering Approach. McGraw-Hill, 2001. ISBN 978-0072549041. 
[18] T.H. Lim, R.H. Song, D.R. Shing, J.I. Yang, H. Jung, I.C. Vinke, and S.S. Yang. Operating characteristics of a $5 \mathrm{~kW}$ class anode-supported planar SOFC stack for a fuel cell/gas turbine hybrid system. International Journal of Hydrogen Energy, 33:1076-1083, 2008.

[19] E. Fontell, T. Kivisaari, N. Christiansen, J.-B. Hansen, and J. Pålsson. Conceptual study of a $250 \mathrm{~kW}$ planar SOFC system for CHP application. Journal of Power Sources, 131:49-56, 2004.

[20] M.H. Halabi, M.H.J.M. de Croon, J. van der Schaaf, P.D. Cobden, and J.C. Schouten. Modeling and analysis of autothermal reforming of methane to hydrogen in a fixed bed reformer. Chemical Engineering Journal, 137:568$578,2008$. 